\title{
Manuel de recherche opérationnelle en matière de planification familiale
}

Andrew A. Fisher

Population Council

John E. Laing

John E. Stoeckel

Population Council

John Townsend

Population Council

Follow this and additional works at: https://knowledgecommons.popcouncil.org/departments_sbsr-rh

Part of the Demography, Population, and Ecology Commons, Family, Life Course, and Society Commons, Health Services Research Commons, International Public Health Commons, and the Medicine and Health Commons

How does access to this work benefit you? Let us know!

\section{Recommended Citation}

Fisher, Andrew A., John E. Laing, John E. Stoeckel, and John Townsend. 1998. "Manuel de recherche opérationnelle en matière de planification familiale." New York: Population Council. 


\section{Manuel de}

recherche opérationnelle en matière de planification familiale seconde édition Andrew A. Fisher John E. Laing John E. Stoeckel John W. Townsend 

Manuel de recherche opérationnelle en matière de planification familiale seconde édition

\author{
Andrew A. Fisher \\ John E. Laing \\ John E. Stoeckel \\ John W. Townsend
}

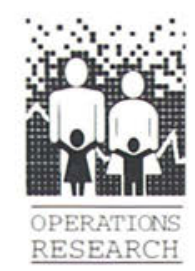

(1) Population Council 
Population Council

One Dag Hammarskjold Plaza

New York, New York 10017

www.popcouncil.org
Library of Congress

Cataloging in Publication Data

Handbook for family planning operations research

design / Andrew A. Fisher ... [et al.] - 2nd ed.

p. $\mathrm{cm}$.

Rev. ed. of: Handbook for family planning

operations research design/Andrew Fisher,

John Laing, John Stoeckel.

Includes bibliographical references

ISBN 0-87834-060-2

1. Birth control-Research-Handbooks, manuals, etc.

2. Operations research-Handbooks, manuals,

etc. I. Fisher, Andrew, 1941- . II. Fisher,

Andrew, 1941-Handbook for family planning

operations research design.

Toute reproduction totale ou partielle est permise sans l'autorisation, ou des auteurs, ou du Population Council, à condition que les reproductions soient distribuées à des fins non lucratives. Il est nécessaire avant toute reproduction commerciale d'obtenir l'autorisation du Population Council. Les auteurs apprécieraient un exemplaire de la documentation dont le texte fut en partie emprunté du Manuel de recherche operationelle.

Imprimé aux Etats-Unis d'Amérique
Andrew A. Fisher, Sc.D., est Associé Principal et Directeur du Projet de Recherche Opérationnelle et d'Assistance Technique en Afrique (Africa OR/TA Project), The Population Council, Nairobi (Kenya).

John E. Laing, Ph.D., est Consultant, Austin, Texas et, dans le passé, Associé Principal du Population Council en Asie Méridionale et Orientale.

John E. Stoeckel, Ph.D., est Associé Principal, The Population Council, Bangkok (Thaïlande).

John W. Townsend, Ph.D., est Associé Principal et Haut Représentant du Population Council en Amérique Latine et dans les Caraïbes, Mexico (Mexique).

Irène Baraket, traductrice de ce livre, est française et vit à Tunis, 11, rue du Niger (Tunisie). 
Préface à la seconde édition Remerciements vii

ix
C Justification du projet de recherche
12
1 Introduction

A Qu'est-ce que la recherche opérationnelle?

B Domaines spécifiques et objectifs de la recherche opérationnelle opérationnelle

1 Etudes exploratoires/diagnostiques

2 Etudes d'intervention sur le terrain

3 Etudes d'évaluation

D Méthodes et procédés de recherche opérationnelle
1

1

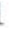

2

\section{2}

3 Choix d'une stratégie pour résoudre le problème

A Qualité des soins/solution du problème 15

B Exemples de stratégies à tester 15

C Principes de base pour le choix de la meilleure stratégie à tester $\quad 16$

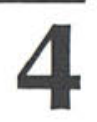

4 Objectifs et hypothèses $\quad 19$

A Objectifs à long terme 19

B Objectifs immédiats 20

C Hypothèses 21

2 Identification et exposé du problème; justification du projet de recherche

A Identification du problème

B Exposé du problème

1 Incidence et prévalence

2 Zones géographiques concernées

3 Types de population concernées

4 Causes probables du problème

5 Solutions possibles

6 Questions non résolues

5 Description détaillée du programme d'intervention

A Qui sera chargé du programme d'intervention?

B Où les activités liées à l'intervention auront-elles lieu? 25

C Quelles activités seront menées? 25 


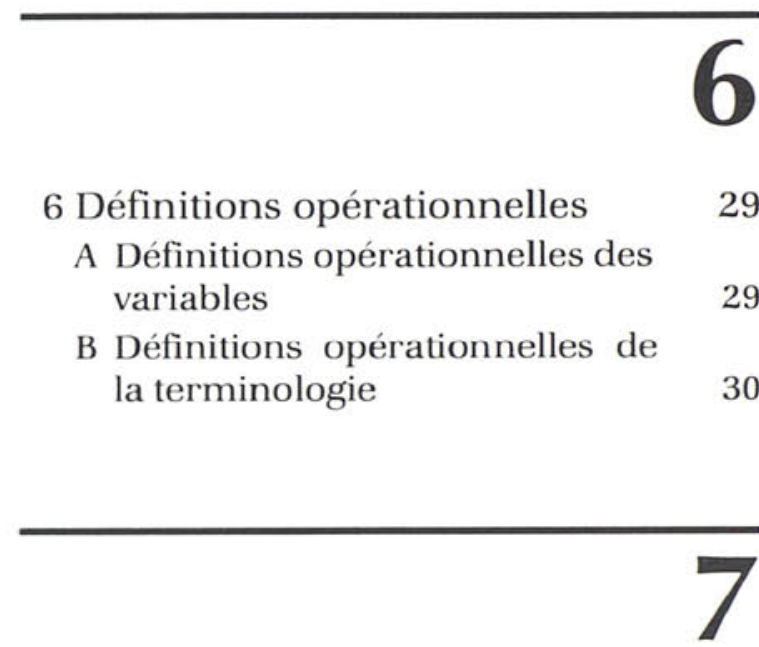

7 Procédés de recherche

A Données fiables et justes

B Sources d'erreurs

1 Effet de conjoncture

2 Effet de sélection

3 Effet de répétition

4 Effet du support de collecte

5 Effet de durée

6 Sortie du champ d'observation

C Procédés de recherche

1 Procédés non expérimentaux

a Test final seulement

b Pré-test et test final

c Test final avec un groupe témoin

2 Procédés expérimentaux

a Pré-test et test final avec un groupe témoin

b Test témoin avec un groupe témoin

3 Procédés quasi-expérimentaux

a Série chronologique

b Groupe témoin non identique

c Echantillons indépendants pour le pré-test et le test

D Choix d'un procédé de recherche

1 Aspects ethiques

2 Contraintes pratiques et administratives

3 Aspects techniques

E Principes de base pour un "bon" procédé de recherche

F La règle des trois multiples
8 Echantillonage

44

A Domaines de recherche 44

B Méthodes de sondage

1 Sondage probabiliste

a Sondage aléatoire simple

b Sondage systématique

c Stratification

d Sondage par grappe

e Sondage à plusieurs degrés

f Sondage avec probabilité proportionnelle à la taille (PPT)

2 Sondage non probabiliste

C Taille de l'échantillon

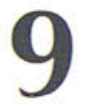

9 Collecte des données

52

A Données quantitatives

1 Interviews structurées

2 Statistiques de services

3 Interviews indirectes

4 Sources de collecte secondaires

B Données qualitatives

1 Interviews non structurées

2 Interviews de groupe (Focus Group Discussion)

3 Observation directe

4 Analyses de contenu de documents

C Contrôle de la qualité des données

D Caractère confidentiel des informations

10 Mise en tableaux et analyse des données

A Tabulation: codage et vérification des données 
B Plan d'analyse des données

1 Caractéristiques des données

a Caractéristiques de tendance centrale

b La variance des données

c Différence entre les résultats

d Relation entre les données

2 Méthodes d'analyse

a Transformation des variables

b Analyse univariée Variables nominales

Variables ordinales

Variables quantitatives

c Analyse des séries chronologiques

d Comparaisons

e Relations bi-variées

Entre variables nominales

Entre variables ordinales

Entre variables quantitatives

f Analyse multivariée

Toutes les variables sont quantitatives

Variables quantitatives et de catégorie

Toutes les variables sont des variables de catégorie

g Analyse coûts-efficacité

h Efficacité de la contraception

i Analyse de la fécondité

C Tabulation fictive

12 Utilisation des résultats de recherche

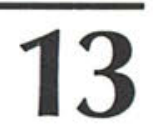

13 Limites de l'étude

75

A Limites du projet de recherche $\quad 75$

B Aspects particuliers $\quad 75$

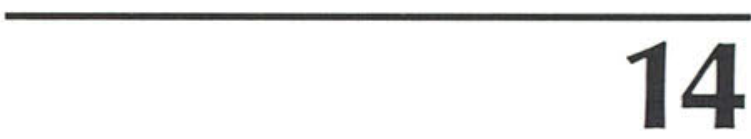

14 Budget de l'étude 76

A Ressources disponibles $\quad 76$

B Le budget de l'étude $\quad 76$

15 Annexes

11 Diffusion des résultats

A Stratégie de diffusion

B Rédaction des rapports de recherche
71

71

16 Page de garde et sommaire

79

71
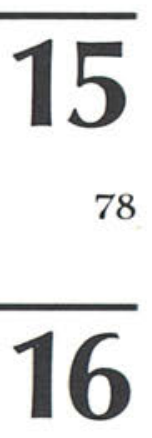

78 



\section{Préface à la seconde édition}

Le Manuel de Recherche Opérationnelle, publié pour la première fois en anglais par le Population Council en 1984, se basait sur l'expérience que ses auteurs avaient acquise lors de la mise en oeuvre de leurs projets de recherche en Asie. Par la suite, ce document a été traduit en français, en espagnol, en thaïlandais, en bahasa Indonésie, en arabe et en chinois. Il a donc été très utilisé non seulement en Asie mais aussi en Amérique Latine et en Afrique.

La seconde édition du Manuel se présente sous la même forme, mais quelques chapitres ont été remaniés et développés. Des exemples concernant l'Afrique et l'Amérique Latine ont été ajoutés là où cela semblait nécessaire. Le chapitre d'introduction comprend un exposé actualisé et plus complet sur le processus de la recherche opérationnelle en matière de santé et planification familiale. D'autre part, de nouveaux chapitres concernant le choix du type d'intervention à tester dans une recherche opérationnelle ainsi que les principaux éléments constituant un programme d'intervention ont été introduits dans le Manuel. Enfin, le chapitre sur la diffusion de l'information a été développé d'une manière plus approfondie et un nouveau chapitre sur l'utilisation des résultats de recherche a été ajouté.

L'utilisation du Manuel suppose une certaine familiarité avec la terminologie et les concepts de la recherche et des statistiques ainsi qu'une certaine expérience en ce qui concerne les études sur le terrain. Le Manuel a été conçu selon plusieurs objectifs. Il vise avant tout à aider les chercheurs en matière de santé et de planification familiale à concevoir et rédiger un projet de recherche détaillé. Il a été souvent utilisé comme manuel de référence dans des ateliers de travail portant sur l'élaboration détaillée et la rédaction correcte d'un projet de recherche. Il vise aussi à aider les administrateurs et directeurs de programme à mieux appréhender le principe de la recherche opérationnelle et les possibilités d'utilisation des résultats de recherche pour l'amélioration des prestations de services. Enfin, bien que le Manuel ne soit pas un document sur les méthodes de recherche, il n'en est pas moins un exposé exhaustif des concepts et des méthodes clés pour la mise en oeuvre des études sur le terrain. 



\section{Remerciements}

Le Manuel a été écrit à l'origine en tant que document de travail pour la formation sous les hospices de US Agency for International Development (USAID) No. DPE-0632-00-1029-00, Recherche Opérationnelle en Matière de Planification Familiale en Asie. Cette nouvelle édition a été rédigée dans le cadre du contrat sous l'égide du Département Population de l'USAID No. DPE-3030-Z-008065-00, Stratégies pour l'Amélioration des Prestations de Service en Matière de Planification Familiale, Projet de Recherche Opérationnelle et d'Assistance Technique en Afrique (Africa OR/TA Project), et du contrat No. DPE-3030-Z-00-9010-00: Projet de Recherche Opérationnelle pour l'Amélioration de la Planification Familiale en Amérique Latine et dans les Caraïbes, Recherche Opérationnelle et Assistance Technique en Amérique Latine et dans les Caraïbes (INOPAL).

Les auteurs remercient tout particulièrement les nombreux participants à des ateliers de travail ainsi que d'autres utilisateurs du Manuel en Asie, en Afrique, et en Amérique Latine pour les commentaires très utiles qu'ils nous ont fait parvenir. Nous sommes reconnaissants aussi pour les conseils, l'appui, et les encouragements de nos collègues à l'USAID et ceux de différentes agences de coopération tels que l'université Columbia, l'université du Michigan, l'université John Hopkins, l'université Tulane et l'université de Hawai. Pour cette nouvelle édition,les auteurs ont tenu compte autant que possible de ces commentaires en évitant toutefois de changer trop profondément la forme du document d'origine. Enfin, nous remercions vivement nos amis du Population Council pour l'aide qu'ils nous ont apportée sous la forme de suggestions et de conseils: Ian Askew, Beverly Ben Salem, John Bongaarts, George Brown, Maria Helena Casanova, Hervé Ludovic de Lys, Mamadou Diallo, James Foreit, Joanne Gleason, Dale Huntington, Anrudh Jain, Michael Koenig, Federico Leon, Tony Martin, Margaret McEvoy, Robert Miller, Cecilia Ndeti, Lewis Ndhlovu, José Garcia Nuñez, Sandra Rosenhouse, Pauline Russell-Brown, Diouratie Sanogo, Valeda Slade, Placide Tapsoba, Luis Varela et Ricardo Vernon. 



\section{Introduction}

\section{A Qu'est-ce que la recherche opérationnelle?}

Les programmes nationaux de santé et de planification familiale en Asie, en Afrique, et en Amérique Latine ont pour but de modifier aussi bien les taux de natalité et de mortalité que la morbidité. Ces programmes revêtent une importance considérable du point de vue de leur coût, de l'effectif du personnel engagé, de la couverture géographique et enfin de la diversité des activités. Ils nécessitent une programmation précise à tous les niveaux, une coordination entre les différentes activités, de la formation adéquate et de la supervision du personnel ainsi que de l'évaluation continue de l'état d'avancement du programme et de son impact. Or, tous ces éléments reposent sur la recherche opérationnelle, ce qui explique l'importance de cette dernière.

La recherche opérationnelle est un processus, une méthode visant à identifier et à résoudre les problèmes inhérents au programme. La recherche opérationnelle telle qu'elle est appliquée actuellement dans les programmes de santé, de planification familiale et autres programmes de développement peut être définie comme un processus continu comprenant essentiellement cinq phases: 1) identification du problème et diagnostic, 2) choix de la stratégie à adopter, 3) expérimentation de la stratégie choisie et évaluation, 4) diffusion de l'information et 5) utilisation de l'information. Le processus de la recherche opérationnelle vise à améliorer le degré d'efficacité, le rendement et la qualité des services fournis par les organismes prestataires ainsi que le degré de disponibilité, d'accessibilité et d'acceptabilité des services du point de vue des utilisateurs.

\section{B Domaines spécifiques et objectifs de la recherche opérationnelle}

La recherche opérationnelle intéresse en premier lieu les activités quotidiennes ou "interventions" des programmes de santé et de planification familiale. Ces interventions effectuées sous la direction des administrateurs et directeurs de programmes touchent divers domaines tels que la formation, la logistique, l'information et l'éducation, ainsi que les services fournis par les cliniques et les systèmes de prestation de services en milieu rural. La recherche opérationnelle s'occupe des problèmes rencontrés par la partie prestation de service du programme; par contre, elle ne s'intéresse pas à l'aspect demande de ces services. En fait, la recherche opérationnelle se caractérise par le fait même qu'elle s'interesse avant tout aux problèmes rencontrés par le système de prestation de services existant. Elle se caractérise aussi par son objectif qui est de rechercher les solutions adéquates ou bien, pour reprendre la terminologie de la recherche, les variables qui peuvent être manipulées au moyen d'une action dans le cadre du programme.

La recherche opérationnelle en matière de santé et de planification familiale fournit des réponses aux problèmes identifiés au niveau du programme. L'un des objectifs essentiels de la recherche opérationnelle est de fournir aux directeurs et administrateurs de programme ainsi qu'aux hauts responsables les informations nécessaires en vue d'améliorer les activités de prestation de services existantes et de programmer la mise en place de nouvelles activités. La recherche opérationnelle a pour but de trouver des solutions pratiques à des situations problèmatiques ainsi que des alternatives viables en remplacement de certaines méthodes de fonctionnement insatis- 
faisantes. Son rôle est de faire le diagnostic et d'évaluer les problèmes rencontrés par le programme; c'est aussi de faire la comparaison entre deux approches différentes concernant les prestations de services en termes d'impact, de rapport coût-efficacité et, du point de vue de la clientèle, en termes de qualité et d'acceptabilité.

\section{Catégories de recherche opérationnelle}

Les études de recherche opérationnelle peuvent être classées selon trois catégories:

1 Etudes exploratoires/diagnostiques. Ces études ont pour objectif de déterminer les paramètres liés à un problème concernant les prestations de services. Elles examinent les facteurs liés au système de prestation des services, et qui ont un impact sur le degré de rendement, d'efficacité et de qualité de ces services. Les études exploratoires/diagnostiques sont de nature restropective ou transversale. On a recours à ce type d'étude chaque fois qu'un problème inhérent au programme a été perçu alors qu'on n'en connaît pas du tout la nature. Ces études se caractérisent essentiellement par la recherche des variables qui peuvent être manipulées par le programme.

2 Etudes d'intervention sur le terrain. Ces études sont destinées à tester, sur une base expérimentale, de nouvelles approches visant à résoudre un problème rencontré par le programme. Dans de nombreux cas, les facteurs à l'origine d'un problème sont connus; par contre, on ne connait pas le meilleur moyen de remédier à ce problème du point de vue de l'efficacité et de la rentabilité. Ces interventions sur le terrain servent à tester de nouvelles approches de prestation de services. Ces études sont toujours de nature prospective ou longitudinale et elles ont recours le plus souvent à une méthodologie de recherche soit expérimentale soit quasi-expérimentale.

3 Etudes d'évaluation. Il arrive très souvent que des activités dans le domaine de la santé et de la planification familiale soient effectuées durant des années sans jamais être évaluées. Dans ce cas, les études d'évaluation peuvent constituer un type de recherche opérationnelle très utile afin d'examiner d'une manière rétrospective ou transversale l'impact du programme.

Ces trois types de recherche opérationnelle ne sont pas mutuellement exclusives. Il arrive souvent que, pour une seule recherche opérationnelle, l'étude commence par une phase exploratoire/diagnostique afin d'identifier les principales variables clés. Il se peut aussi que la deuxième phase mette en place une intervention sur le terrain qui sera suivie par la suite d'une phase évaluation afin de déterminer l'impact de l'intervention.

\section{Méthodes et procédés de recherche opérationnelle}

Les méthodes de recherche opérationnelle vont de l'étude qualitative à l'étude quantitative, alors que les procédés de recherche vont de l'étude non-expérimentale à l'étude expérimentale. Cependant, il n'existe pas de méthodes ou de procédés de recherche spécifiques à la recherche opérationnelle. En fait, ce qui distingue la recherche opérationnelle, ce n'est pas l'application d'un ensemble particulier de méthodes ou de procédés de recherche mais plutôt le thème ou l'objectif de cette recherche. En d'autres termes et pour simplifier, l'objectif de la recherche opérationnelle est d'améliorer le système de prestation des services. Alors que la recherche opérationnelle peut utiliser des procédés expérimentaux ou non-expérimentaux, en ayant recours à l'analyse quantitative de phénomènes démographiques ou à l'examen qualitatif de certains aspects liés à la santé, l'objectif principal est toujours d'obtenir une meilleure compréhension du "fonctionnement" du programme afin que les améliorations nécessaires puissent être apportées. 


\section{Exemples de thèmes faisant l'objet de recherches opérationnelles}

Des centaines de recherches opérationnelles ont été conduites en Asie, en Afrique, et en Amérique Latine. Voici, pour illustrer ce qui précède, une série de neuf axes de recherche:

1 Programmes de formation. Un très grand nombre d'agents de santé à travers le monde a reçu une formation. Il est donc fréquent que des études opérationnelles soient menées pour examiner le contenu et les méthodes de formation, ou pour comparer deux approches différentes en termes de niveau de connaissance et d'efficacité du personnel de santé.

2 Information, éducation et communication (IEC). Les activités d'information de la population constituent une partie essentielle de la plupart des programmes de santé et de planification familiale. Il arrive fréquemment que les études de recherche opérationnelle soient conçues dans le but de comparer différentes approches en matière de communication en terme de compréhension du message et durabilité de son impact, coûts-efficacité et capacité à atteindre les différents types d'audience ciblée.

3 Systèmes d'observation et de surveillance (SOS). Tout programme de santé et de planification familiale collecte des informations sur la clientèle et les services fournis. Cependant, parfois, ces informations manquent de précision ou bien, tout simplement, elles ne sont pas utilisées au niveau de la gestion administrative. Des études de recherche opérationnelle peuvent être conçues avec pour objectif l'expérimentation de nouveaux systèmes d'information et le test de nouvelles procédures facilitant l'utilisation des informations recueillies pour l'amélioration des services.

4 Impact du programme. Les études de recherche opérationnelle servent souvent à tester d'une manière restropective et sur le terrain l'impact des différents types de systèmes de prestation de services tels que l'utilisation du système de distribution communautaire ou le recours aux sagesfemmes traditionnelles pour l'assistance des femmes en couche. L'impact peut être mesuré de différentes façons en évaluant, par exemple, la prévalence contraceptive, les taux de continuation de la contraception, le niveau de satisfaction de la clientèle, et l'amélioration du degré de disponibilité et de l'accessibilité des services.

5 Administration et gestion. L'évaluation qualitative de l'administration et de la gestion du programme font parfois l'objet d'études de recherche opérationnelle de type diagnostic.

6 Qualité des soins. Un nombre croissant de recherches opérationnelles se sont données comme objectif d'évaluer la qualité et l'accessibilité des services fournis à la clientèle. Ces études peuvent fournir une analyse de la situation actuelle en matière de santé et de planification familiale.

7 Réseaux de distribution privés. De nombreux programmes de planification familiale utilisent les détaillants privés, tels que les pharmacies, comme moyen de distribution des contraceptifs délivrés sans ordonnance. Les études de recherche opérationnelle peuvent servir à comparer les deux systèmes de distribution publique et commercial.

8 Le SIDA et la planification familiale. Dans de nombreux pays, les responsables de programmes s'inquiètent de l'impact éventuel du SIDA sur les activités liées à la planification familiale. En Afrique et en Amérique Latine, un certain nombre de recherches opérationnelles ont été conduites afin de tester l'intégration de l'information et de l'éducation en ce qui concerne le SIDA dans les activités courantes de planification familiale.

9 Implication des hommes dans la planification familiale. Dans certains programmes nationaux de planification familiale, l'attention s'est portée presque exclusivement sur les femmes, et la plupart des services ne sont d'ailleurs fournis que dans les centres de santé maternelle et infantile. De nombreuses recherches opérationnelles ont donc été menées afin de tester différentes approches visant aussi bien à impliquer les hommes dans les programmes de planification familiale qu'à leur fournir des services appropriés sur les lieux du travail ou ailleurs.

Il est évident qu'il existe bien d'autres thèmes pouvant faire l'objet de recherches opérationnelles. Quelque soit le thème choisi, il est important de rappeler que l'objectif essentiel de la recherche opérationnelle n'est pas de recueillir des informations et d'identifier l'origine du problème mais bien d'utiliser ces informations pour le résoudre. En fait, mettre au point puis mener une recherche opérationnelle ne constitue qu'une étape par rapport à l'ensemble du processus de recherche. La diffusion des résultats de l'étude pour améliorer les prestations de services constitue une phase essentielle du processus de recherche opérationnelle. 


\section{Identification et exposé du problème; justification du projet de recherche}

\section{A Identification du problème}

Le déroulement des programmes de santé et de planification familiale soulèvent de nombreux problèmes facilement décelables. Cependant, il est parfois délicat d'en identifier un pour en faire l'objet d'un projet de recherche. L'une des conditions essentielles pour effectuer des études opérationnelles est d'identifier puis d'explorer un problème donné. $\mathrm{Si}$, pour le chercheur lui-même, le thème reste imprécis, il est évident que cela se répercutera sur le contenu de la proposition de recherche; dans le cas contraire, il peut aboutir à une bonne formulation des objectifs et des hypothèses de recherche, des variables principales à étudier ainsi que des principes méthodologiques pour mesurer l'incidence de ces variables.

La mise en oeuvre de tout projet de recherche suppose l'existence d'un problème particulier décelé à travers l'observation d'une difficulté donnée, d'une situation jugée insatisfaisante ou encore d'une discordance entre les résultats attendus et les faits observés. A la base de toute recherche se trouve nécessairement un problème, ce qui ne veut pas dire que toute difficulté nécessite un projet de recherche. Un projet de recherche existe si les trois conditions suivantes sont réunies:

1 Existence d'une discordance entre les résultats attendus et les faits observés.

2 Interrogation sur les causes de cette discordance.

3 Possibilité d'au moins deux réponses cohérentes à cette interrogation.

Ce dernier point est particulièrement important. En effet, s'il n'y a qu'une réponse possible et cohérente à cette interrogation, il n'y a pas lieu de mener une recherche spécifique. Soit l'exemple suivant.

\section{Exemple d'une situation non propice à une recherche}

Exposé du probleme: Les résultats d'une enquête dans le District $\mathrm{A}$ avaient montré l'existence de 1000 femmes utilisant d'une manière continue la pilule; mais les statistiques de services pour le mois précédent montrent qu'aucune de ces femmes n'est en train d'utiliser cette méthode.

Discordance: Les 1000 femmes auraient dû être en train d'utiliser la pilule mais tel n'est pas le cas.

Interrogation: Quel(s) facteur(s) se trouvent à l'origine de la rupture par ces 1000 femmes de l'utilisation de la pilule?

Réponse: Des inondations dues à la mousson ont empêché l'approvisionnement du District A en pilules et toutes les réserves sont épuisées.

L'exemple qui précède montre qu'il n'est pas nécessaire de mener une recherche spécifique sur la rupture de l'utilisation de la pilule puisque, si les faits sont exacts, la réponse à l'interrogation est d'ores et déjà connue. Par contre, il apparaît nécessaire de mener une étude sur les défaillances 
du système logistique et d'approvisionnement et sur son incapacité d'assurer la fourniture des moyens contraceptifs au District A pendant la période de la mousson. Soit l'exemple suivant.

\section{Exemple d'une situation propice à une recherche}

Exposé du problèmè: Le District $A$ subit toujours des inondations pendant la période des moussons. Ayant pris conscience du problème, les responsables du programme national de planification familiale ont mis en place un nouveau système logistique pour l'approvisionnement de ce district. Chaque ménage utilisant la pilule reçoit, avant la période des moussons, une réserve de contraceptifs pour quatre mois. Pendant la période des moussons, de légères embarcations à moteur sont disponibles pour l'approvisionnement des centres accessibles au personnel de planification familiale travaillant dans les villages. Malgré ces nouvelles mesures, les statistiques de services ont montré pour cette année l'absence de pilules disponibles dans le District A.

Discordance: Le nouveau système logistique devrait assurer un approvisionnement continu en pilules; mais cette année on a observé qu'il n'y avait pas de pilules disponibles.

Interrogation: Pourquoi le nouveau système logistique s'est-il révélé incapable de fournir les pilules aux ménages qui les utilisent?

Réponses possibles: 1 Une demande de réapprovisionnement en pilules n'a pas été faite à temps avant les pluies.

2 Les embarcations utilisées pour le transport des pilules sont en pannes.

3 Le personnel de terrain n'a pas été averti de la mise en place du nouveau système et n'a donc pas fourni aux ménages utilisateurs de pilules la réserve nécessaire pour quatre mois.

Dans cet exemple, plusieurs réponses sont possibles dont une ou plusieurs peuvent être correctes. La situation est par conséquent propice à une recherche opérationnelle.

Dans certains cas, il est relativement facile d'identifier le problème, d'en exposer les données, d'émettre des hypothèses sur les causes possibles et de dégager finalement celles qui reflètent le plus la réalité. Dans l'exemple précédent, les causes qui tiennent à l'approvisionnement et au système logistique peuvent être faciles à dégager et ne nécessiteraient pas une recherche coûteuse. Par contre, d'autres problèmes sont nettement plus délicats à identifier et à étudier, comme on le voit dans l'exemple suivant:

\section{Exemple d'une situation propice à une recherche}

Exposé du problème: Une enquête récente en matière de planification familiale a mis en évidence l'existence d'importantes disparités entre les villes en ce qui concerne les taux d'utilisation de la contraception. Ces taux varient de $6 \%$ à $80 \%$ d'un village à l'autre alors que tous bénéficient du même niveau de prestation de services de planification familiale et de santé.

Discordance: Tous les villages devraient avoir le même taux d'utilisation de la contraception, alors qu'il existe en fait des variations importantes d'un village à l'autre.

Interrogation: Quels sont les facteurs qui expliquent les disparités régionales en matière de taux d'utilisation de la contraception?

Réponses possibles: 1 La réalité socio-économique diffère d'un village à l'autre. Dans certains villages, c'est l'agriculture qui prédomine; dans d'autres, 
c'est la pêche. Certains villages sont Hindous, d'autres en majorité Musulmans ou Boudhistes. Certains ont facilement accès à la ville, alors que pour d'autres, l'accès est trés difficile ou pratiquement impossible; certains sont pourvus d'écoles, de dispensaires, d'électricité, d'eau courante, alors que d'autres ne bénéficient pas de ces avantages. Ces différences socioéconomiques ont une influence sur le taux d'utilisation de la contraception.

2 Le soutien à la planification familiale diffère d'un village à l'autre. Dans certains villages, les personnalités locales soutiennent fortement le programme national de planification familiale, ce qui n'est pas le cas dans d'autres. Dans certains villages, il existe des associations féminines qui soutiennent la planification familiale, dans d'autres non. Ces différences dans le soutien au programme de planification familiale influencent le niveau d'utilisation de la contraception.

3 L'efficacité du personnel de santé et de planification familiale varie selon les villages. Certains sont très motivés et actifs dans leurs secteurs, alors que d'autres le sont moins. Ces différences concernant l'efficacité du personnel de terrain influencent le niveau d'utilisation de la contraception.

Alors que les données du problème décrit ci-dessus sont assez claires, les raisons en paraissent complexes. Trois causes ont été fournies et on peut certainement en ajouter d'autres. Dans ce cas-là, le chercheur doit consacrer ses efforts à l'identification du problème avec comme objectif d'en dégager les aspects les plus importants.

\section{Exemple d'une situation propice à une recherche}

Exposé du problème: Les superviseurs observent lors de leurs visites régulières de la clientèle dans le cadre d'un programme communautaire de planification familiale que de nombreuses utilisatrices de la pilule sont généralement mal informées de la manière dont cette méthode doit être utilisée. Par exemple, $58 \%$ des femmes interrogées avaient pris la pilule d'une manière incorrecte durant le mois précédent. Certaines avaient attendu trop ou pas assez longtemps après le cycle menstruel; d'autres ne savaient pas ce qu'elles devaient faire après avoir oublié de prendre la pilule.

Discordance: L'une des fiertés du programme de planification familiale est de fournir des services de qualité. La majorité des agents de terrain pour la promotion de la santé chargés d'informer et d'approvisionner la clientèle ont reçu trois jours de formation lors de leur recrutement. De plus, ils sont supervisés lors de leur ré-approvisionnement. Toutes les utilisatrices devraient donc savoir utiliser correctment la méthode de leur choix, mais il est clair qu'il y a un manque de connaissance.

Interrogation: Comment la direction du programme peut-elle assurer un niveau acceptable en ce qui concerne i'utilisation correcte des méthodes dans le programme communautaire en milieu rural?

Réponses possibles: 1 La procédure de sélection des candidats aux postes d'agents volontaires pour le développement du milieu rural est inadéquate. Cette procédure de sélection doit être modifiée et peutêtre changée. 
2 Le programme de formation de trois jours en vigueur est inadéquat tant au niveau du contenu qu'au niveau de la pratique en matière de communication avec la clientèle. Ce programme de formation devrait être revu.

3 Le système de supervision accorde autant de temps à chacun des agents de terrain quelque soient les besoins de la communauté et les compétences de l'agent. Le système de supervision doit être réexaminé.

4 En recrutant des agents de terrain dont le niveau de compétence est très variable, la direction du programme néglige la qualité du programme communautaire. Une composante qualité des soins doit être introduite dans le programme.

5 La gamme des services dont l'agent de terrain est chargé est trop variée et complexe. La gamme des services fournis doit être modifiée.

Dans cet exemple, les données du problème sont claires. Les responsables du programme de planification familiale ont observé que de nombreuses utilisatrices de la pilule sont mal informées sur la façon d'utiliser cette méthode. La discordance réside dans le fait que les utilisatrices de la pilule devraient savoir mais ne le savent pas. La discordance entre ce qui devrait être et la réalité suscite une interrogation et cinq réponses au problème exposé. On ignore quelles sont les réponses correctes ou celles qui sont les meilleures possibles.

L'un des meilleurs moyens facilitant la mise en oeuvre de la première phase d'une recherche opérationnelle, c'est-à-dire l'identification du problème, est d'impliquer tout au long du processus de recherche non seulement les chercheurs mais aussi les administrateurs (et d'autres encore, selon le cas, tels que les chefs de village, les instituteurs et les dirigeants locaux). Une telle implication a l'avantage d'allier l'expérience et les points de vue du personnel administratif et des dirigeants locaux aux compétences méthodologiques et techniques des chercheurs.

Inclure dans une même équipe des chercheurs et administrateurs de programme constitue pour chacun une expérience professionnelle fructueuse car elle dépasse le cadre restreint de la conception et de l'exécution d'une seule recherche opérationnelle. Les chercheurs prennent alors conscience des problèmes quotidiens rencontrés par les administrateurs en ce qui concerne l'organisation des prestations de services. Cette compréhension approfondie peut faciliter la mise au point d'une étude sur certains aspects qu'il est possible de modifier. Les administrateurs de leur côté, se rendent compte de la nécessité d'identifier et de cerner les problèmes rencontrés par le programme sur la base d'informations fiables. Ils considèrent alors la recherche comme un outil important pour la prise des décisions. La recherche devient aussi pour eux un processus permanent à laquelle ils peuvent contribuer. Enfin, leur participation dès le début aux diverses phases de la recherche opérationnelle les rendra sûrement d'autant plus motivés en ce qui concerne l'utilisation des résultats.

\section{B Exposé du problème}

L'identification du problème constitue une première étape importante dans la conception de l'étude et doit être suivie par une description détaillée en termes de fréquences, d'intensité, de répartition et de tous les indices pour lesquels les données sont disponibles. L'objectif consiste à rassembler toutes les informations connues concernant le problème et de ses causes qu'il est toujours possible de deviner mais sur lesquelles il arrive souvent de faire erreur ce qui risque de fournir une base fragile au projet de recherche. Le meilleur moyen d'arriver au but est de passer en revue toute la documentation appropriée, d'examiner les statistiques de services disponibles, de discuter avec des personnes qui connaissent le sujet et de rechercher dans les théories économiques, sociales et sanitaires les causes probables de la situation. L'examen des différentes sources de données relatives au sujet et une investigation de type épidémiologique permettent au chercheur de préciser les points suivants: 
1 Incidencé et prévalence. Une investigation de type épidémiologique devrait toujours être faite, ce qui peut fournir une idée sur l'ampleur du problème, sa répartition et sa fréquence. Un tel diagnostic aide le chercheur à definir les différents paramètres.

2 Zones géographiques concernées. Il est essentiel de connaïtre si certaines zones géographiques sont particulièrement concernées. Ce problème n'affecte-t-il que les zones rurales? Existe-t-il aussi dans les villes? Ou bien le trouve-t-on uniquement dans les zones montagneuses, côtières ou insulaires?

3 Types de population concernés. Le problème touche-t-il certains groupes spécifiques de la population tels que les mères, les enfants, les adolescents, les jeunes couples ou les femmes de 36 ans?

4 Causes probables du problème. L'analyse des données disponibles devrait dégager un certain nombre de causes possibles. Quelles sont les idées les plus courantes concernant la situation? Existe-t-il une opinion largement partagée sur les causes du problème ou au contraire des avis opposés?

5 Solutions possibles. Des tentatives ont pu déjà être faites pour surmonter les difficultés. Quels types de solution ont été déjà essayés dans le passé et avec quel degré de succès? Quelles sont les approches qui semblaient les plus adaptées et celles qui ne l'étaient pas?

6 Questions non résolues. Quels sont, à partir des informations disponibles sur le problème, les aspects qui restent inconnus et les questions qui méritent des investigations supplémentaires?

L'examen des connaissances déjà acquises sur le même type de problème constitue une démarche fondamentale dans le processus de recherche. Une analyse sérieuse des informations disponibles permet de saisir l'importance du problème du point de vue social, économique, politique et sanitaire. Cela permet aussi de dégager les concepts théoriques et les variables essentielles que d'autres chercheurs ont déjà considéré comme importants. Il en est de même pour les hypothèses de recherche à tester. Enfin, cela évitera au chercheur de s'engager dans des types d'étude déjà réalisés maintes fois dans le passé et qui ont fourni des résultats assez comparables.

\section{Exemple d'une identification et exposé d'un problème de recherche: Asie}

1 Bien que d'importants progrès aient été accomplis ces dix dernières années en ce qui concerne les taux de fertilité dans les pays du Sud-Est Asiatique, certains aspects négatifs subsistent dans les programmes, ce qui pourrait freiner la tendance à la baisse de ces taux. En Indonésie, le taux relativement élevé de la prévalence de $42 \%$ à Java et Bali doivent être considérés en tenant compte des taux relativement faibles dans les régions des Isles Périphériques I et II-respectivement de $17 \%$ et $6 \%$ seulement. Aux Philippines, les méthodes modernes de contraception ne comptent que pour $25 \%$ de la pratique contraceptive. En Malaisie, les acceptrices de la stérilisation ne constituent que $6 \%$ du nombre total des nouvelles acceptrices et les nouvelles utilisatrices de DIU ne comptent que pour $3 \%$. En Thaïlande, le programme de vasectomie ne progresse pas, alors que d'excellents taux d'acceptation ont été enregistrés après que le secteur privé fait parvenir ce type de service jusque dans les villages.

2 La plupart des responsables des programmes en Asie savent qu'il est nécessaire d'intensifier les activités et de fournir des efforts supplémentaires si l'on veut maintenir les tendances établies au cours des années 70. En Indonésie, il faut prêter attention non seulement aux îles périphériques mais aussi aux zones urbaines, notamment à Djakarta. Cette ville possède un système de prestation de services de santé et de planification familiale qui doit couvrir en principe tous les quartiers, et pourtant la proportion de contraceptrices parmi les femmes mariées n'est que de 21 pourcent, soit environ deux fois moins que dans l'ensemble de Java et de Bali. Certaines autres zones urbaines du pays sont également en retard par rapport aux zones rurales.

3 Bien qu'on ne sache pas à quels facteurs attribuer les différences entre les proportions de contraceptrices des zones urbaines et des zones rurales en Indonésie, on pense que les 
zones urbaines ont perdu les structures traditionnelles d'organisation et de direction communautaires qui appuient le programme de planification familiale dans les zones rurales. Une autre raison des différences observées pourrait être le fait que les habitants, notamment ceux qui viennent de s'installer dans les zones urbaines, ne savent pas où obtenir les contraceptifs. Parmi les nouveaux migrants, beaucoup ont un faible niveau d'instruction, n'ont pas accès aux sources d'information et ne connaissent pas l'emplacement des centres de santé et de planification familiale. Souvent aussi, le personnel du planification familiale en milieu urbain peut difficilement desservir à domicile de façon continue les nouveaux migrants, car ceux-ci ont tendance à déménager fréquemment d'un quartier à l'autre. Il est possible que ces déménagements conduisent les ménages à abandonner, ou du moins à interrompre leur pratique de la contraception.

4 Des tentatives ont déjà été faites à plusieurs reprises afin d'augmenter la pratique contraceptive dans les villes en rapprochant les services de la population dans les quartiers périphériques. On a distribué des condoms et des pilules dans les petits commerces telles que les boutiques et échoppes. Bien que ces approches aient été utiles, elles ont par ailleurs de grands inconvénients. Les équipes mobiles coûtent cher et ne peuvent atteindre qu'un nombre relativement faible de clients potentiels. Les petits commerces sont commodes pour les personnes déjà utilisatrices, mais ils ne constituent pas un moyen très efficace pour attirer de nouveaux utilisateurs et ils ne peuvent pas non plus offrir les services nécessaires en matière de santé maternelle et infantile telles que les vaccinations et les consultations pré et post-natales.

5 Ce qu'il faut dans les zones urbaines, c'est une approche capable (1) d'atteindre un grand nombre d'usagers actuels et potentiels, et (2) de leur offrir toute une gamme de services de santé et de planification familiale. Comment réaliser cela dans les meilleures conditions, voilà une question importante et non résolue pour le programme national de planification familiale.

6 L'une des approches déjà proposée, mais qui n'a pas été tentée jusqu'à présent, consiste à former une catégorie de personnel de santé et de planification familiale en milieu urbain qui contacterait la clientèle actuelle et potentielle sur le lieu de travail. Avec l'aide des employeurs et des syndicats, ces agents de santé et de planification familiale pourraient créer des centres de services dans les usines, les administrations, les hôtels, les chantiers, les marchés et autres lieux de travail. Il est probable que cette approche serait moins coûteuse que l'utilisation d'équipes mobiles; elle permettrait non seulement d'atteindre de très nombreuses personnes, mais aussi d'offrir une gamme de services beaucoup plus large que celle fournie par les petits commerces.

\section{Commentaires}

Dans le premier paragraphe de l'exemple ci-dessus, on identifie grossièrement le problème. Plusieurs pays d'Asie du Sud-Est ont réussi à réduire fortement leur taux de fécondité, mais les programmes se heurtent à des obstacles qui empêchent les progrès de se poursuivre. On présente alors plusieurs de ces obstacles. Ces exemples servent à indiquer quelles sont les zones concernées.

Dans le deuxième paragraphe, l'étude de ces obstacles est limitée à un seul pays, l'Indonésie. L'attention se limite également à un seul aspect: La différence qui existe entre les taux de pratique de la contraception dans les zones urbaines et dans les zones rurales. On fait état de la discordance suivante: Djakarta a un système de prestation des services de santé et de planification familiale conçu pour couvrir la ville entière. Théoriquement, ce système devrait conduire à ce que la proportion de contraceptrices soit égale à celle des zones rurales. Pourtant cette proportion à Djakarta est deux fois plus faible que celle de l'ensemble de Java et de Bali. A la fin du deuxième paragraphe, nous savons quel est l'aspect particulier à approfondir: la différence de pratique contraceptrice entre villes et campagnes. Nous connaissons la zone géographique concernée: l'Indonésie, les zones urbaines, notamment Djakarta. Nous connaissons l'ampleur du problème: 21 pourcent de contraceptrices à Djakarta au lieu de 42 pourcent dans l'ensemble de Java et Bali. Nous savons aussi quelle est la population concernée: la population en milieu urbain. 
Dans le troisième paragraphe, on avance plusieurs causes possibles à ces difficultés mais on ne sait pas laquelle d'entre elles correspond le plus à la réalité.

Le quatrième paragraphe suggère plusieurs solutions au problème du faible taux d'utilisation de la contraception dans les zones urbaines: les équipes mobiles et les petits commerces. Chacune de ces approches, quoique utiles, se sont révélées inadéquates.

Le cinquième paragraphe suggère ce qu'il faut faire pour résoudre le problème, et le sixième paragraphe propose une nouvelle approche qui n'a pas encore été expérimentée.

\section{Exemple d'une identification et exposé d'un problème: Afrique}

1 Au Burkina Faso, un programme de visites effectuées par des volontaires a été mis en place il y plusieurs années dans le but d'éduquer les couples en matière de planification familiale et de les orienter vers les centres de prestation de services appropriés. Le programme avait été conçu en tant qu'expérimentation d'une approche possible, destinée surtout au milieu urbain, pour la mise en place de la politique gouvernementale en matière de planification familiale. Le recours à des contraceptrices volontaires, satisfaites de la méthode qu'elles utilisent, pour effectuer les visites à domicile dans leur voisinage et parler de la planification familiale ainsi que de l'existence des services avait été jugé plus efficace pour atteindre les couples concernés que l'utilisation des mass-média ou l'emploi à plein temps de sages-femmes rénumérées.

2 Bien que certaines volontaires se soient montrées assez actives en ce qui concerne le recrutement de nouvelles contraceptrices, d'une manière générale, le programme n'a pas obtenu les résultats escomptés. Une étude diagnostic a mis en relief les difficultés suivantes:

a Alors que l'utilisation de volontaires pour effectuer les visites à domicile est manifestement moins onéreuse que l'emploi de sages-femmes rénumérées à plein temps, cela ne signifie pas que l'efficacité soit la même. En effet, la motivation des volontaires pour effectuer des visites à domicile régulières est très variable. D'une manière générale, la motivation initiale, proche de l'enthousiasme, s'évanouit peu à peu et après quelques mois, les volontaires ne visitent plus que très peu de ménages sinon plus du tout.

b L'aire géographique assignée à chacune des volontaires, un Secteur en général, est trop étendue pour être couverte correctement par une seule personne. Les volontaires ont tendance à effectuer les visites à domicile d'une manière non méthodique, sans coordination ni calendrier pré-établi.

c La supervision des volontaires sur le terrain est inadéquate. Ceci peut être la raison du manque d'enthousiasme de certains d'entre eux.

d Bien que les volontaires aient reçu une formation de base en matière de planification familiale, aucun recyclage permettant de renforcer leurs compétences n'est prévu.

e D'après le témoignage de plusieurs couples ayant été contactés par les volontaires, les informations fournies durant ces visites à domicile sont plutôt superficielles. Il semble que les volontaires soient incapables de fournir les informations spécifiques sur les méthodes demandées par les utilisatrices potentielles.

f Le suivi fait défaut. Un ménage ayant été visité une fois ne reçoit presque jamais d'autres visites.

3 Tous ces problèmes ont été largement discutés par les responsables du programme. On ne sait pas quel problème ou quelle combinaison de ces problèmes affecte le programme de visites à domicile. Cependant, en se basant sur une étude diagnostic, plusieurs solutions paraissent possibles:

a Il serait possible de se passer de tout ce personnel volontaire et de le remplacer par des sages-femmes rénumérées à plein temps. Il est certain que ces sages-femmes consacreraient plus de temps aux visites à domicile et qu'elles seraient capables de fournir une information plus détaillée à la clientèle. D'un autre côté, l'emploi de sages-femmes augmenterait les coûts du programme des visites à 
domicile, ce qui, à long terme, rendrait difficile son maintien. De plus, il est peu probable que l'on puisse recruter un si grand nombre de sages-femmes puisqu'un nombre relativement peu important de cette catégorie de personnel a été formé.

b Une seconde alternative consisterait à reprendre la formation des volontaires. Bien que cette solution serait sûrement moins coûteuse que l'emploi des sagesfemmes, il est probable que tous les problèmes ne seraient pas résolus. Le secteur attribué à chacune des volontaires serait encore trop étendu pour être couvert correctement. Il faudrait alors instituer un programme de supervision sur le terrain. Le suivi des clientes resterait un problème, et même si une nouvelle formation des volontaires améliorait leur niveau de connaissance, il est peu probable qu'elles puissent fournir des informations aussi détaillées que les sages-femmes.

c Une troisième alternative serait de supprimer le programme des visites à domicile et compter sur les campagnes médiatiques ainsi que sur les réunions de la population concernée au niveau du Secteur pour l'informer au sujet de la planification familiale. Alors qu'une telle approche contribuerait à informer la population en matière de planification familiale, il est peu probable que cela suffirait à changer les comportements d'un manière significative. De nombreux documents relatifs à la communication démontrent clairement que le changement des comportements est la plupart du temps dû à la communication interpersonnelle, plutôt qu'aux campagnes médiatiques. Ainsi, une telle approche ne favoriserait donc pas le recrutement d'un grand nombre de nouvelles acceptrices.

d Une quatrième approche consisterait à combiner les meilleurs aspects des trois approches décrites ci-dessus. Par exemple, on peut concevoir un programme ayant les objectifs suivants: 1) attribuer à des acceptrices volontaires, satisfaites de la méthode qu'elles utilisent, une aire géographique plus réduite dont il serait plus facile de s'occuper; 2) les faire travailler selon un plan de travail et un calendrier pré-établi à raison d'un certain nombre de fois par semaine et accompagnées de sages-femmes rénumérées; 3 ) assurer aux volontaires un appui, une supervision et un recyclage régulier; et enfin 4) organiser des réunions de groupes au niveau du Secteur sur la planification familiale. Les volontaires pourraient alors contribuer à contacter des amis, des parents et des voisins intéressés par les services de planification familiale. Les sages-femmes pourraient superviser les volontaires et fournir à la clientèle potentielle une information plus détaillée et spécifique aussi bien sur la planification familiale que sur la santé maternelle et infantile.

\section{Commentaires}

Le premier paragraphe ne concerne qu'un seul pays, le Burkina Faso; il s'agit d'un programme prévu pour une seule ville, Ouagadougou, dont l'objectif est de fournir aux couples concernés des services d'éducation et de contraception plus accessibles grâce à l'utililisation de volontaires. On considérait à l'origine qu'un tel programme serait plus efficace que l'emploi des mass-média et des sages-femmes rénumérées.

Le deuxième paragraphe relève les données d'une situation posant un problème, une discordance entre les résultats escomptés (un programme de volontaires fonctionnant correctement) et les résultats obtenus (un programme rencontrant des problèmes). Une étude diagnostic récente a mis en relief pour ce programme de visites à domicile six problèmes différents.

Le troisième paragraphe note que ces problèmes ont été largement discutés sans qu'on ne connaisse lequel ou bien quelle combinaison de plusieurs d'entre eux sont les plus importants. Plusieurs solutions en vue d'améliorer le programme sont alors présentées. Les trois premières alternatives sont rejetées pour différentes raisons. La quatrième solution, qui est une combinaison de certains éléments tirés des trois premières, est adoptée comme étant celle ayant le plus de chance de réussir. C'est donc cette dernière alternative qui sera testée dans une recherche opérationnelle sous la forme d'un programme d'intervention sur le terrain. 


\section{En résumé-identification et exposé du problème}

1 Pour l'identification et l'exposé du problème, les étapes suivantes sont à suivre:

a En premier lieu, décrire succintement la situation.

b Enrichir l'exposé à partir de la documentation disponible et approfondir l'étude du problème.

c Porter l'attention plus particulièrement sur les aspects les plus importants se prêtant à la recherche.

2 Faire un premier essai d'identification du problème en adoptant le plan suivant:

Exposé du problème: (Rédaction en quelques lignes exposant simplement la situation)

Discordance: (Faire état de la discordance entre les résultats attendus et les faits observés)

Interrogation: (Poser la question fondamentale)

Réponses possibles: (Avancer deux ou trois réponses possibles)

3 Enrichir l'exposé par des éléments relatifs au sujet à partir de la documentation existante, des statistiques de services disponibles, de l'opinion de certains spécialistes, ainsi que de toute autre source d'information. Vérifier que certains concepts théoriques ou certaines variables n'ont pas été omis et en établir une liste. Répondre si possible aux questions suivantes:

a Incidence et prévalence du problème?

b Zones géographiques concernées?

c Types de populations concernés?

d Conclusions des recherches déjà menées à ce sujet?

e Les approches déjà tentées pour résoudre le problème?

f Degré de succès de ces tentatives?

g Principales questions non résolues?

4 Reprendre à nouveau l'exposé identifiant la situation en intégrant tous les éléments réunis à partir de la documentation disponible et des autres sources. Suivre le plan: exposé de la situation, discordance, interrogation, réponses possibles. Bien délimiter le problème et ne négliger aucun détail susceptible d'éclairer la situation tout en restant clair et précis.

5 D'autres personnes devraient lire le document ainsi préparé afin de tester la clarté et la précision de l'exposé relatif au problème à étudier. Si l'aspect discordant entre les résultats attendus et les faits observés n'est pas évident pour le lecteur, il est préférable de tout reprendre à zéro!

\section{Justification du projet de recherche}

Une fois le problème identifié et exposé, il reste au chercheur de fournir les arguments nécessaires afin de démontrer l'importance du projet de recherche. Cette justification est d'autant plus nécessaire que la recherche exige souvent du temps et des moyens matériels importants. Le chercheur doit être à même de prouver la nécessité de l'étude et d'en démontrer la pertinence.

\section{Exemple justifiant le choix d'un sujet de recherche: Asie}

En Indonésie, la différence des taux d'utilisation de la contraception entre le milieu rural et le milieu urbain provient essentiellement du taux élevé d'accroissement démographique en milieu urbain ainsi que de l'inadéquation du système de prestation de services et de son inefficacité. Dans toute l'Asie du Sud et du Sud-Est, l'accroissement démographique en milieu urbain est nettement plus sensible qu'en milieu rural, soit en moyenne 4 à 8 pourcent contre 2 à 3 pourcent, en raison essentiellement de l'exode rurale et de l'importance de la natalité. Les migrants qui se déplacent vers les villes à la recherche d'un travail et d'une vie meilleure, se retrouvent en fait dans des conditions sociales, économiques et sanitaires pire que celles qu'ils ont quittées. Dans la plupart des grandes villes, les moyens efficaces pour assurer entre autres des prestations de services de santé font encore défaut. 
Aussi bien le gouvernement que la direction du programme de planification familiale sont conscients de la différence qui existe dans les taux d'utilisation de la contraception entre Djakarta et les zones rurales du fait de l'inadéquation du système de prestation de services en matière de santé et de planification familiale et de son inefficacité. De nouvelles approches plus adaptées aux conditions de vie des habitants en milieu urbain doivent être trouvées et expérimentées. Les migrants nouvellement installés et les couches sociales les plus deshéritées de la population doivent être atteints en priorité, vu que pour la majorité d'entre eux, les services de santé et de planification familiale ne sont ni disponibles ni accessibles. Les différences observées entre les taux d'utilisation de la contraception dans le milieu rural et ceux observés dans le milieu urbain persisteront tant que durera cette situation.

\section{Commentaires}

Les arguments présentés pour la justification du projet de recherche se situent à plusieurs niveaux. On reconnait en premier lieu que les différences d'utilisation de la contraception entre le milieu rural et le milieu urbain caractérisent non seulement l'Indonésie mais aussi une grande partie de l'Asie du Sud et du Sud Est, ce qui veut dire, en d'autres termes, que cet aspect est largement répandu. On montre en deuxième lieu que le problème continuera de s'aggraver au fur et à mesure de l'accroissement démographique des villes. En troisième lieu, on indique que la direction du programme de planification familiale est préocupée par la situation. En quatrième lieu, c'est un problème lié à l'inadéquation du système existant de prestation des services de santé et de planification familiale en milieu urbain et à son inefficacité. Enfin on affirme que si de nouvelles approches en matière de prestation de services de santé de base et de planification familiale ne sont pas mises en place, les différences observées continueront à exister.

\section{Exemple justifiant le choix d'un sujet de recherche: Afrique}

De nombreux programmes de planification familiale attachent moins d'importance aux centres urbains qu'au milieu rural et ce, malgré l'urbanisation rapide dans presque tous les pays d'Afrique. Par ailleurs, les programmes qui ont été mis en place dans les cités n'ont pas toujours fourni de bons résultats. Les résidents des centres urbains ayant généralement un meilleur niveau d'instruction ainsi qu'un niveau de vie plus élevé, on en conclut, souvent à tort, qu'ils ont une meilleure connaissance en matière de planification familiale ainsi qu'un meilleur accès aux services de santé et de planification familiale.

Au Burkina Faso, le nombre de pauvres ne fait qu'augmenter. Il est urgent de développer de nouvelles approches novatrices en vue de toucher cette population. Il est tout à fait irréaliste de compter pour la prestation des services d'éducation et de planification familiale sur les quelques centres hospitaliers ou cliniques de Ouagadougou, d'une part, parce qu'ils sont destinés à la médecine curative et qu'ils sont, d'autre part, submergés de patients. Il est irréaliste aussi de considérer que la population urbaine est disposée, plus que la population rurale, à dépenser du temps, de l'argent et de l'énergie afin de rejoindre les centres de santé où les services ne sont pas toujours disponibles. Les services de planification familiale et les activités d'éducation doivent être disponibles sur place. Des programmes de quartier, similaires aux progammes de distribution communautaire dont l'efficacité et la durabilité à été prouvé, doivent être développés dans les zones urbaines.

\section{Commentaires}

L'exposé justifiant le choix d'un sujet de recherche souligne que les programmes de planification familiale dans de nombreux pays Africains n'attachent pas assez d'importance aux centres urbains. En d'autres termes, le problème de l'insuffisance des services de planification familiale pour les résidents en milieu urbain est un problème très répandu qui ne concerne pas un seul pays. Ce problème est aussi d'actualité au Burkina Faso où le nombre de pauvres ne fait qu'augmenter. Enfin, la justification du sujet de recherche met en relief le manque d'équité dans la répartition des efforts. Une attention similaire à celle qui à été accordée au milieu rural en développant les programmes de distribution communautaire doit étre portée maintenant sur le milieu urbain au niveau des quartiers. 


\section{En résumé-justification du projet de recherche}

1 Pour la justification du projet de recherche, il est important de répondre aux questions suivantes:

a Le problème est-t-il d'actualité? Se pose-t-il d'une manière urgente?

b Est-il très répandu? Concerne-t-il de nombreuses régions?

c Concerne-t-il plus particulièrement certains types de population, tels que les mères et les enfants?

d La situation est-elle liée aux activités du programme en cours?

e La situation est-elle liée aux conditions économiques, sociales et sanitaires générales, telles que le chômage, les inégalités de revenus, la condition féminine, la scolarisation et la santé maternelle et infantile?

f Qui d'autre est préoccupé par le problème? les dirigeants du pays? les médecins? etc.

2 Se servir des réponses pour justifier le projet de recherche. 


\section{Choix d'une stratégie pour résoudre le problème}

\section{A Qualité des soins et solution du problème}

Une fois que le problème faisant l'objet d'une recherche opérationnelle a été identifié, une certaine variété de stratégies pour le résoudre sont possibles. Il arrive souvent que ces stratégies n'impliquent qu'une modification à apporter aux prestations de services existants ou bien le test d'une nouvelle approche concernant l'organisation de ces services, plutôt que la mise en place de nouvelles structures de prestation de services.

Il est assez fréquent de constater que de nombreux problèmes, et par conséquent les stratégies potentielles qui visent à les résoudre, sont liés à l'aspect qualité des soins. Lorsqu'on met au point une stratégie appropriée destinée à résoudre un problème spécifique, il est toujours utile de se référer aux six éléments relatifs à l'aspect qualité des soins qui sont les suivants:

1 Elargir la gamme des méthodes contraceptives fournies à la clientèle dans les cliniques, les hôpitaux, les points de distribution commerciaux et communautaires.

2 Fournir les informations à la clientèle d'une manière claire et précise en ce qui concerne les différentes méthodes disponibles en spécifiant leurs avantages et leurs inconvénients.

3 Améliorer les compétences techniques des prestataires de services.

4 Encourager la quantité et la qualité des relations inter-personnelles clientèle/prestataires de services.

5 Encourager la continuité de l'utilisation de la contraception.

6 Fournir une configuration appropriée des services à la clientèle.

\section{B Exemples de stratégies à tester}

Les stratégies spécifiques traitant des éléments liés à la qualité des soins ou à d'autres problèmes faisant l'objet de recherches opérationnelles sont bien sûr très nombreuses. Par exemple, parmi les centaines de recherches opérationnelles effectuées en Asie, en Amérique Latine et en Afrique, différentes stratégies ont été développées et testées dans le but d'atteindre les objectifs suivants:

- Elever le degré de disponibilité et d'accessibilité des services de planification familiale en utilisant les détaillants privés, les lieux de travail, les cliniques locales ainsi que les responsables des dépôts de contraceptifs au niveau des villages et en assouplissant les heures d'ouverture des services de prestation.

-Améliorer l'information, l'éducation et la communication en associant les mass-média à plus de communication inter-personnelle dans le cadre de cours d'éducation pour adultes, de réunions de groupes au niveau des villages, d'activités de conseil et ce, avec le concours des acceptrices satisfaites de leur méthode.

- Introduire ou promouvoir des méthodes contraceptives particulières en employant d'autres types de personnel telles que des infirmières pour l'insertion du DIU ou du 
NORPLANT ${ }^{\circledR}$; en créant des cliniques pour les hommes afin de promouvoir la vasectomie; en organisant des réunions d'éducation pour les associations de femmes afin d'encourager l'allaitement maternel et la contraception naturelle.

- Améliorer le rendement du personnel par l'amélioration des procédures et des critères de recrutement, une formation basée sur la compétence, une définition réaliste des tâches à effectuer, une supervision modulée et donc plus efficace, et enfin, de meilleures conditions de travail.

- Renforcer le rendement du programme par la formation en matière de gestion administrative, une meilleure circulation de l'information en ce qui concerne les prises de décisions et la programmation des activités; par l'utilisation de la microinformatique, l'amélioration des services statistiques sur le plan fiabilité et disponibilité; par des systèmes de gestion des ressources financières ainsi que des systèmes de logistique plus efficaces et performants; et enfin, par une meilleure maintenance du matériel, des véhicules et autres équipements.

- Satisfaire les besoins d'autres groupes cibles de la population tels que les hommes, les femmes sur les lieux de travail, les couches défavorisées en milieu urbain, les groupes ethniques ou religieux en adaptant les supports IEC ainsi que les services à ces groupes.

- Augmenter le degré de recouvrement des frais et assurer le financement à long terme du programme par la participation du secteur privé, la tarification des produits et des services de marketing social, la contribution des employeurs pour les services fournis, la contribution des administrations locales, la couverture sociale et enfin par les entreprises semi-privées.

- Intégrer les services de planification familiale dans les autres programmes de santé tels que les programmes de vaccination, de promotion de l'allaitement maternel ou d'autres activités liées aux soins de santé primaires.

\section{Principes de base pour le choix de la meilleure stratégie à tester}

Avant de déterminer quelle stratégie liée à la prestation des services ou à la gestion administrative sera testée dans le cadre d'une recherche opérationnelle, il est nécessaire, tout d'abord, de revoir en détail les données du problème en question. C'est la nature du problème qui devrait déterminer le choix de la stratégie à tester. En d'autres termes, le meilleur choix en matière de stratégie est celui qui apporte une solution au problème rencontré par le programme. Cela semble être évident, mais il est surprenant de constater que souvent, une seule stratégie telle que le marketing social ou l'utilisation de plus de supports IEC (affiches, videos, brochures et dépliants) est considérée comme une panacée et par conséquent, fréquemment mise en oeuvre pour résoudre n'importe quel problème, alors que cette stratégie n'est pas forcément la plus adéquate.

En plus du fait qu'il est nécessaire de revoir les données du problème, il y a d'autres principes de base qu'il est utile de suivre afin de choisir et de mettre en oeuvre la stratégie à tester dans le cadre d'une recherche opérationnelle.

1 Examiner les stratégies utilisées par d'autres personnes afin de résoudre des problèmes similaires. Pour cela, il suffit d'étudier les résultats fournis par un grand nombre d'études en matière de planification familiale effectuées dans le passé. Il est essentiel de trouver, en ce qui concerne la gestion administrative ou les prestations de sevices, quelle stratégie semble la meilleure pour la solution de problèmes spécifiques.

2 Tenir une réunion avec les personnes les plus concernées ou les plus préoccupées par le problème. Les personnes directement concernées par les problèmes de gestion et de prestation des services ont parfois une idée très claire des stratégies possibles pouvant être utilisées pour surmonter le problème. Il est toujours très important de recueillir leur avis car la plupart du temps, ce sont eux qui, mieux que personne, sont capable de proposer les meilleures stratégies.

3 Rechercher des stratégies dont la mise en oeuvre n'entraîne pas une surcharge préjudiciable au fonctionnement du programme. Si les resources requises pour 
tester une stratégie sont si importantes qu'elles ont une incidence sur les activités courantes de prestation de services, cette stratégie ne devrait pas être mise en place.

4 Choisir une stratégie qui peut être maintenue à long terme. Même si une certaine stratégie est très efficace à court terme, c'est-à-dire durant la phase d'intervention d'une recherche opérationnelle, si elle ne peut être maintenue à long terme (dans la période qui suit la fin de la recherche opérationnelle) par une structure adéquate, cette stratégie a fort peu de chance de résoudre le problème. Il est essentiel de rechercher toujours des stratégies dont le financement pourra être maintenu.

5 Rechercher des stratégies faciles à mettre en place. Lorsqu'on doit choisir entre deux stratégies touchant les prestations de services et dont l'impact potentiel de chacune est équivalent, il faut choisir celle qui est la plus facile à mettre en place en fonction du matériel et des équipements requis, du temps et des coûts, des moyens logistiques et organisationnels ou d'autres considérations semblables.

6 Developper des stratégies dont les solutions dépendent directement de la direction du programme. Les solutions suggérées par la stratégie choisie, c'est-à-dire les "variables indépendantes" selon la terminologie de la recherche, doivent être sous le contrôle direct de la direction du programme de planification familiale.

7 Eviter les stratégies dont le test sur le terrain entraîne des coûts plus élevés que les bénéfices escomptés. Il est évident qu'une stratégie de recherche opérationnelle ne devrait pas être testée si les bénéfices escomptés sont insignifiants par rapport aux efforts requis pour sa mise en place.

8 Eviter les stratégies qui ne sont pas compatibles avec les objectifs et le programme de l'organisme d'exécution. Une stratégie rencontrera une forte résistance et ne bénéficiera pas des ressources et de l'attention nécessaires pour une mise en oeuvre réussie si elle ne peut s'intégrer dans les objectifs généraux et le programme d'activités de l'organisme d'exécution concerné.

\section{Exemple de stratégie pour résoudre un problème: Amérique Latine}

Le pourcentage relativement important des utilisatrices de la pilule ne sachant pas comment l'utiliser correctement est un problème directement lié à au moins deux éléments relatifs à la qualité des soins, ce sont: 1) l'information fournie à la clientèle; et 2) les relations inter-personnelles clientèle-prestataires de services.

Lorsque 58 pourcent des contraceptrices orales prennent la pilule d'une manière incorrecte, il est clair qu'elles sont mal informées. Soit les prestataires de services ne leur fournissent pas les informations nécessaires, soit la relation clientèle-prestataires de services n'est pas bonne, ce qui explique une communication inefficace de l'information.

Dans une telle situation, on peut envisager plusieurs stratégies pour résoudre le problème. Premièrement, on peut éditer des supports IEC sous forme de brochures, d'affiches et de films vidéo sur l'utilisation de la méthode orale et faire en sorte d'atteindre l'ensemble de la clientèle, ancienne et nouvelle, avec ces nouveaux supports. Une seconde stratégie serait de revoir et de modifier le programme de formation des agents de santé en milieu rural. Les agents déjà en activité pourraient par exemple bénéficier d'un recyclage. Ce recyclage mettrait l'accent sur l'acquisition des techniques de communication permettant de fournir à la clientèle une information complète sur tous les aspects de la prise de la pilule. C'est cette dernière stratégie, avant tout autre, qui sera mise en place, testée et évaluée.

Les principales raisons ayant conduit à choisir une stratégie de recyclage sont les suivantes:

a Des études de recherche opérationnelle récentes effectuées en Amérique Latine montrent clairement que la communication inter-personnelle entre la clientèle et les prestataires de services qui se tient dans une atmosphère de compréhension, de respect et d'honnêteté et qui donne lieu à un échange de questions et de réponses est plus efficace que la transmission impersonnelle de cette information par les mass-média.

b Le programme de planification familiale a déjà une composante formation et recyclage des agents de santé en milieu rural. Il sera donc plus facile de remanier le contenu du 
programme de formation et de recycler l'ensemble des agents concernés sur une période de 18 mois que de mettre au point, tester et éditer de nouveaux supports IEC.

c La stratégie du recyclage constitue une approche facile à financer car les coûts initiaux et ultérieurs sont bien moins élevés que pour la stratégie qui consiste à de nouveaux supports IEC.

\section{Commentaires}

Dans l'exemple ci-dessus, le problème de la prise incorrecte de la pilule est liée aux éléments relatifs à la qualité des soins. Etant donné ces éléments, deux stratégies possibles visant à résoudre le problème sont envisagées. La première consisterait à fournir à la clientèle l'information sur la prise de la pilule par la voie médiatique (brochures, dépliants, films vidéo), et la deuxième reposerait sur la communication inter-personnelle entre les prestataires de services et la clientèle. C'est la seconde stratégie qui est choisie comme celle qui sera mise en place, testée sur le terrain et évaluée. Les trois raisons justifiant ce choix sont les suivantes: 1) des études de recherche opérationnelle dejà faites montrent qu'elle est plus efficace, 2) organiser un recyclage est plus facile que de produire de nouveaux supports IEC, et 3) cette stratègie est moins coûteuse et a par conséquent plus de chance d'être maintenue à long terme.

\section{En résumé-choix d'une stratégie à tester}

Après l'identification, l'exposé et la justification d'un problème assez important pour faire l'objet d'une recherche, il est nécessaire de choisir une stratégie appropriée afin de le résoudre. Il faut tout d'abord ré-examiner la nature du problème en question. Ce problème est-il lié à l'aspect plus général de la qualité des soins, et si cela est le cas, quelle(s) stratégie(s) peut-on envisager par rapport aux six éléments relatifs à la qualité des soins?

Se référer alors aux principes de base pour le choix d'une stratégie puis, pour l'élaboration du projet de recherche, suivre la démarche suivante:

1 Identifier les stratégies possibles qui pourraient être appliquées pour résoudre le problème.

2 Indiquer clairement quelle(s) stratégie(s) éventuelle(s) vous avez choisie(s) comme étant la/les plus appropriée(s) pour résoudre le problème.

3 Justifier la/les stratégie(s) adoptée(s) en indiquant:

a La réussite d'une telle stratégie dans le passé pour résoudre un problème similaire.

b La simplicité d'exécution de cette stratégie par rapport à d'autres qui auraient probablement le même impact.

c Les potentialités humaines et financières pour maintenir cette stratégie une fois que la recherche opérationnelle serait terminée. 


\section{Objectifs et hypothèses}

Le thème central de l'étude est devenu à ce stade bien précis. L'identification, l'exposé et la justification du projet de recherche précédemment décrits débouchent normalement sur la formulation des objectifs spécifiques et des hypothèses de recherche. Il s'agit de montrer l'utilité des résultats quant au déroulement du programme et d'annoncer les hypothèses à tester qui décrivent les relations entre les différentes variables.

\section{A Objectifs à long terme}

Pour la plupart des projets de recherche, les conclusions attendues sont présentées comme ayant des répercussions à long terme. Il arrive souvent qu'on présente ces répercussions d'une manière irréaliste, comme par exemple une contribution à la baisse de la mortalité, de la morbidité ou de la fécondité; ce qui est hors de portée d'une étude isolée. Un but plus modeste consisterait à fournir aux responsables ainsi qu'aux dirigeants du pays les éléments d'information susceptibles d'améliorer le système de prestation de services ou de réorienter le programme.

La formulation des objectifs à long terme sert d'une part à placer le sujet dans le contexte économique, social et sanitaire général et de préciser d'autre part les répercussions probables de l'étude. Ce qui justifie d'autant plus la nécessité du projet de recherche. Il faut dégager à cet egard le caractère lointain de ces objectifs et les formuler en conséquence.

\section{Exemples d'objectifs à long terme}

1 Etude des variations régionales de la pratique contraceptive au Bengladesh. "L'objectif à long terme de cette étude consiste à aider la direction du programme à élaborer une nouvelle approche en matière d'éducation à l'intention des villages ayant de faibles taux de prévalence."

2 Etude pour tester une approche visant à fournir les services de planification familiale à la population rurale. "L'objectif à long terme de cette étude est d'expérimenter en milieu urbain un système d'éducation et de prestation de services en matière de santé et de planification familiale qui soit durable, efficace et de qualité."

3 Une étude expérimentale visant à intégrer l'éducation et les services communautaires de planification familiale dans le système de prestation des soins de santé de base dans deux zones rurales au Cameroun. "L'objectif à long terme de cette étude est de mettre en place en milieu rural un système intégré, efficace et rentable de prestation de services en matière de santé et de planification familiale qui aura un impact significatif à la baisse sur le taux de mortalité et de morbidité maternelle et infantile."

4 Enquête sur les connaissances, les attitudes et les pratiques relatives à la planification familiale chez les notables locaux de Sri-Lanka. "L'objectif à long terme de cette étude est de faire participer davantage les notables locaux dans les activités de santé et de planification familiale." 


\section{B Objectifs immédiats}

A la différence des objectifs à long terme qui indiquent ce que l'on souhaite atteindre, les objectifs immédiats constituent ce qui sera effectivement réalisé en relation étroite avec le problème à étudier. Le chercheur présente dans ce cas les variables qui seront mesurées ainsi que les activités qui seront menées.

Dans la plupart des cas et particulièrement dans les évaluations, les objectifs immédiats doivent être exprimés en termes concrets; c'est-à-dire qu'ils annoncent ce qui sera effectivement réalisé en spécifiant par qui, en quel lieu, à quelle date, de quelle manière et pour quelles raisons, etc.

\section{Exemples d'objectifs immédiats}

1 Une étude de recherche opérationnelle en Gambie pour la prestation de services de planification familiale sur les lieux de travail. "Durant la durée de l'intervention sur le terrain, L'Association Gambienne pour la Planification Familliale, qui travaillera avec 15 à 20 usines sélectionnées, conduira une recherche opérationnelle visant à tester deux types de système de prestation de services sur les lieux de travail. On procèdera à la comparaison de ces deux approches en les confrontant l'une à l'autre par rapport à une troisième (témoin), et ce en termes de coûts, de connaissances et de pratique en matière de contraception, de satisfaction des services et autres paramètres."

2 Une étude en Indonésie sur les différences entre les taux de prévalence contraceptive en milieu urbain et rural. "La première phase de cette étude consistera pour le BKKBN Indonésien à conduire une étude exploratoire à Jakarta et dans deux Kécamatans ruraux afin de déterminer les facteurs inhérents à l'organisation administrative et au programme et qui sont à associer avec la variation de la pratique contraceptive parmi les femmes actuellement agées de 15-44 ans."

"Durant la deuxième phase de cette étude, prévue pour 1993, le BKKBN procèdera à l'évaluation d'un nouveau système de prestation de services en termes d'augmentation (a) de la practique contraceptive chez les femmes de 15 à 44, et (b) de l'utilisation des services de soins de santé primaires parmi les couches de la population les moins favorisées."

3 Evaluation de différentes méthodes de formation. "La Division Formation du Projet de Planification Familiale et de Santé Maternelle et Infantile conduira en 1991 une étude d'évaluation pour mesurer en terme d'efficacité la différence entre le personnel de terrain ayant été formé dans un centre de formation et le personnel ayant été formé dans le cadre d'un programme de formation sur le terrain."

4 Enquête sur la connaissance, les attitudes et les pratiques des personnes influentes de la communauté en ce qui concerne la planification familiale. "En 1992, le Ministère du Plan conduira une enquête sur 100 villages sélectionnés d'une manière aléatoire afin de déterminer la connaissance, les attitudes et les pratiques des personnes influentes de la communauté en ce qui concerne la planification familiale."

\section{Commentaires}

Il est à remarquer que dans les exemples cités ci-dessus, les objectifs immédiats sont exprimés en terme d'intentions concrètes de mener une enquête, d'évaluer un programme, etc. Le projet de recherche est aussi placé dans un contexte général. Les responsables de l'étude, ainsi que la période et le lieu sont précisés. Enfin, et c'est l'essentiel, les variables les plus importantes à étudier y sont specifiées à savoir-les facteurs économiques, sociaux, culturels et inhérents au programme, la pratique contraceptive, l'utilisation des soins de santé de base, les performances du personnel de terrain, la formation, etc. Toutes ces variables devront être soigneusement définies plus tard, mais en attendant, elles contribuent à cerner l'objet de la proposition de recherche. 


\section{Hypothèses}

Une hypothèse de recherche suppose l'existence d'une relation entre deux ou plusieurs variables qu'il s'agit de vérifier empiriquement. Les objectifs à long terme annoncent les répercussi'sns possibles des conclusions de l'étude, alors que les objectifs immédiats (formulés en termes concrets) définissent les tâches à réaliser ainsi que les facteurs à mesurer; quant aux hypothèses de recherche, elles suggèrent la relation entre deux ou plusieurs variables.

Ces hypothèses servent fondamentalement à orienter le processus de recherche et à décrire les variables dépendantes et les variables indépendantes qui seront utilisées ainsi que le type d'analyse adopté pour mesurer les relations entre elles.

Une hypothèse donnée peut suggérer que l'effet de la variable A est associé à celui de la variable B ou que l'effet de la variable A est une cause de celui de la variable B. Parfois cette relation est assortie de certaines conditions $\mathbf{X}, \mathbf{Y}$, et $\mathbf{Z}$ par exemple. Il reste que l'attention est attirée sur certaines variables précises. Il est important, à ce propos, de distinguer les variables dépendantes (ou variables à expliquer) des variables indépendantes (ou variables explicatives). Une variable dépendante est celle qui est déterminée ou influencée par une variable indépendante. La relation fondamentale entre les deux types de variables peut être présentée comme l'indique la figure 4.1. Ceci constitue une relation directe entre les deux types de variables. En d'autres termes, la variable dépendante varie en fonction de la variation de la variable indépendante.

Il est toutefois assez rare dans les recherches en matière de planification familiale de trouver un type de relation aussi direct. En effet, dans la plupart des cas, la relation entre les deux types de variables s'effectue d'une manière indirecte par le biais d'autres variables appelées les variables intermédiaires. L'effet de la variable indépendante sur la variable dépendante est, dans ce cas, soit amplifié soit amorti par l'effet des variables intermédiaires parfois designées comme des variables de contrôle. Les chercheurs en matière de planification familiale font presque toujours appel à un schéma comportant des variables intermédiaires (voir figure 4.2)

A titre d'exemple, un programme de planification familiale qui cherche à augmenter le nombre d'acceptrices de méthodes de planification familiale (variable dépendante) peut lancer une vaste campagne d'information, d'éducation et de communication (variable indépendante). Mais un programme d'IEC ne peut à lui seul et en tant que tel assurer l'augmentation du nombre d'acceptrices. Il doit passer par le biais de certaines variables intermédiaires et agir sur elles pour

FIGURE 4.1

Relation directe entre les variables indépendantes et dépendantes
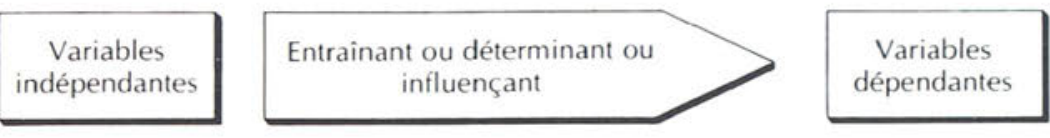

FIGURE 4.2

Variables intermédiaires

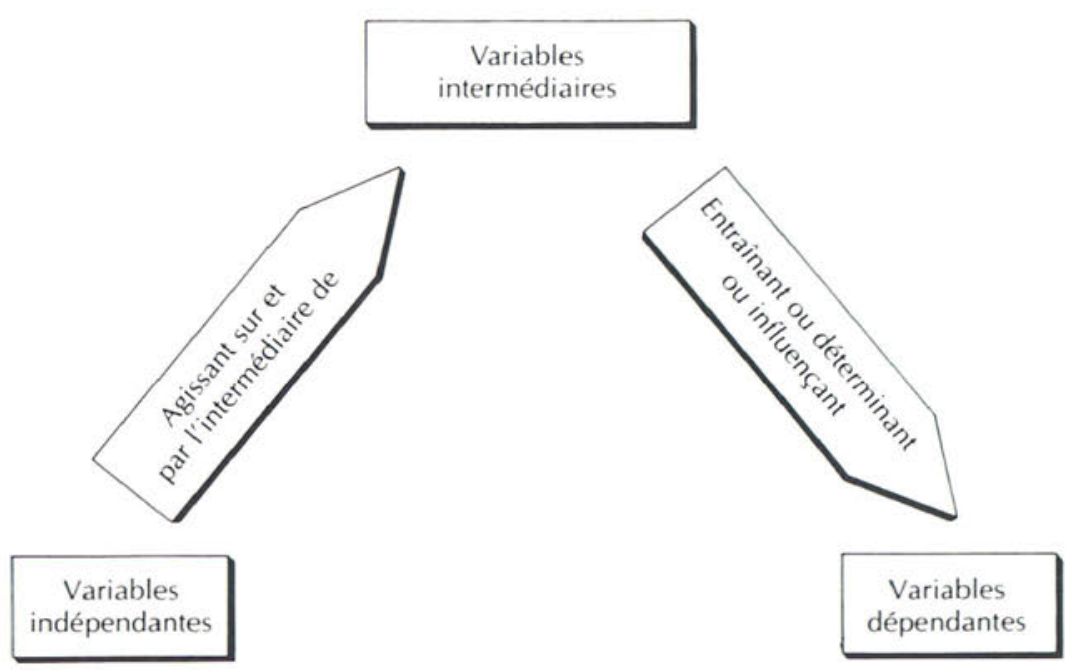


FIGURE 4.3

Influence des variables

intermédiaires sur un

programme IEC

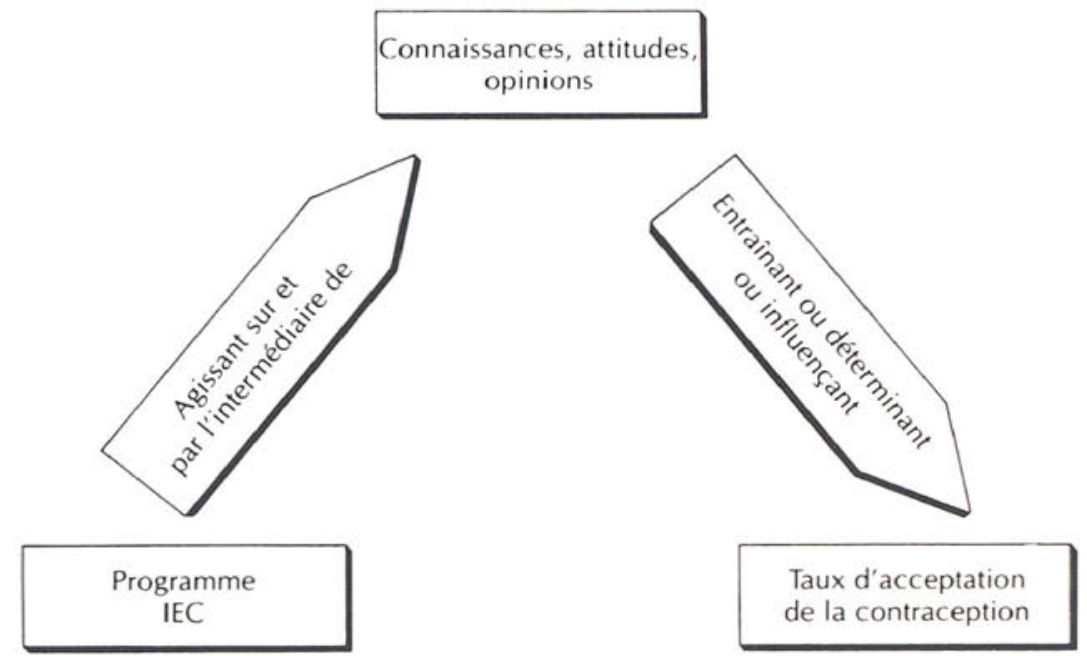

que celles-ci puissent influencer à leur tour le recours aux pratiques contraceptrices. Ces variables intermédiaires sont souvent assez nombreuses, mais le degré de connaissance des méthodes, le type d'attitude envers la contraception et le nombre d'enfants souhaité par les ménages constituent les aspects qui peuvent être les plus sensibles à un programme d'IEC. Le schéma de recherche pour l'évaluation des effets d'un programme d'IEC comme l'indique la figure 4.3.

Dans les recherches opérationnelles en matière de planification familiale, les hypothèses les plus couramment utilisées sont celles qui considèrent les activités du programme comme variable indépendante. C'est ainsi que le contenu du programme tel que les activités d'IEC constitue une variable indépendante. Le but de l'étude est de mesurer le degré d'influence de la variable indépendante sur la variable dépendante, celle-ci étant l'objet même de la proposition de recherche. Les variables intermédiaires contribuent à amplifier ou à amortir le type de relation entre le programme (variable indépendante) et les résultats (variable dépendante).

La plupart des études font intervenir plusieurs variables indépendantes et variables dépendantes. L'énoncé des hypothèses de recherche doit toujours tenir compte de l'existence des relations entre les variables. Il s'agit, en premier lieu, de mettre en relief le problème central de l'étude (variable dépendante); puis de considérer le ou (les) facteur(s) (variables indépendentes) pouvant déterminer ou influencer ce problème. Il s'agit enfin de réfléchir sur les relations entre les variables dépendantes et indépendantes et de voir si les effets se font d'une manière directe ou bien par le biais des variables intermédiaires.

\section{Exemples d'hypothèses:}

1 Les taux de pratique contraceptive devraient être plus élevés dans les villages présentant de nombreux aspects de modernisation que dans les villages qui en ont moins.

2 Les villages où le personnel de planification familiale est crédible devraient avoir des taux de pratique contraceptive plus élevés que dans les villages où le personnel ne l'est pas.

3 Le personnel de santé et de planification familiale qui a suivi une formation de cinq semaines sur le terrain devrait être plus efficace que le personnel qui a suivi une formation théorique de même durée.

4 Une meilleure activité du personnel de terrain devrait favoriser une acceptation plus importante des méthodes de contraception par les ménages.

5 Un programme d'information et d'éducation ayant recours à la radio, à la télévision et à la presse écrite devrait permettre d'élever le niveau de connaissance de la population en matière de planification familiale.

6 La prévalence contraceptive devrait être plus élevée parmi la population qui possède un 
bon niveau de connaissance des méthodes contraceptives que parmi la population ayant un niveau de connaissance plus faible.

\section{Commentaires}

Chacune des hypothèses citées ci-dessus suggère une relation entre deux ou trois variables. Dans la première hypothèse, les aspects de modernisation d'un village (variable dépendante) sont en relation avec la pratique contraceptive (variable indépendante). Dans la seconde hypothèse, la relation est faite entre la crédibilité du personnel de terrain (variable indépendante) et la pratique contraceptive (variable dépendante). La troisième et la quatrième hypothése vont ensemble. Dans la troisième hypothèse, il y a une relation entre un programme de formation sur le terrain de 5 semaines (la variable indépendante) et l'efficacité du personnel (variable d'intervention et aussi variable dépendante). Dans la quatrième hypothèse, l'efficacité du personnel de terrain se trouve être la variable indépendante et les taux d'acceptation deviennent la variable dépendante. Ces deux hypothèses peuvent être regroupées comme l'indique la figure 4.4. De même, les hypothèses 5 et 6 vont ensemble. Le niveau de connaissance des méthodes qui est la variable dépendante dans l'hypothèse cinq, devient une variable dans l'hypothèse six.

En résumé, on doit formuler pour chaque projet d'étude, (1) les objectifs à long terme, (2) les objectifs immédiats et (3) les hypothèses de recherche (exception faite des études purement exploratoires). Chacune de ces rubriques possède sa fonction propre.

1 Les objectifs à long terme indiquent les répercussions possibles et souhaitables de l'étude.

2 Les objectifs immédiats indiquent ce qui sera effectivement réalisé dans le cadre de l'étude.

3 Les hypothèses fournissent des suggestions sur la relation entre deux ou plusieurs variables.

FIGURE 4.4

Deux hypothèses sur l'effet d'un programme de formation du personnel de terrain sur les taux d'acceptation des méthodes contraceptives

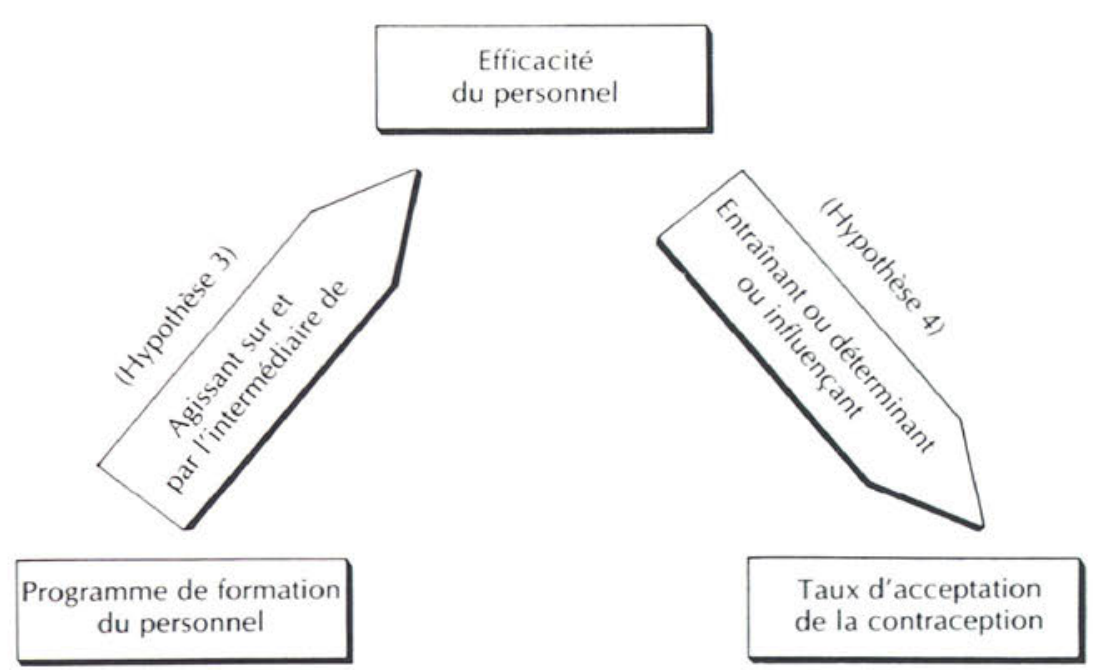




\section{En résumé-objectifs à long terme, objectifs immédiats et hypothèses de recherche}

1 Liste des principales variables à étudier en distinguant les variables dépendantes (ou variables à expliquer) des variables indépendantes (ou variables explicatives). Les variables intermédiaires peuvent être classées selon le cas dans l'une ou l'autre des rubriques. La (les) variable(s) dépendante(s) doivent être liées directement au problème à étudier.

2 Examen des variables dépendantes et rédaction d'un exposé des objectifs à long terme qui s'y rattachent. Se poser la question suivante: "Comment la mise à jour des facteurs qui influencent ou déterminent les (la) variables dépendantes aiderait-elle les directeurs du programme et les responsables?" Dans quelle mesure les résultats de l'étude pourront-ils aider à améliorer le système de prestation de services, à perfectionner les programmes de formation, à élaborer les supports éducatifs? En d'autres termes, quelles seront les répercussions souhaitables de l'étude?

3 Rédaction des objectifs immédiats de l'étude. Se concentrer sur les points concrets et précis, et sur les aspects que l'on doit mesurer en répondant aux questions suivantes:

a Qui sera chargé des activités?

b Quel élément de quelle activité?

c Quelles sont les personnes visées?

d Quand?

e Où?

f Dans quel but?

4 Enfin, rédaction des hypothèses de recherche que l'étude se propose de vérifier. Examiner de nouveau la liste des variables indépendantes et des variables dépendantes, des relations qui existent entre elles et des conditions sous-jacentes à ces relations si elles existent. La rédaction des hypothèses doit être faite sous la forme affirmative en évitant la forme négative. Une hypothèse de recherche doit accompagner chacune des relations importantes à étudier. 


\section{Description détaillée du programme d'intervention}

Si l'étude comprend un programme d'intervention, comme par exemple le test d'une nouvelle méthode de formation ou une comparaison entre deux nouvelles approches concernant la prestation des services, il sera nécessaire de décrire en détail dans le projet d'étude la nature de l'intervention. Il est nécessaire surtout de donner des informations concernant les questions posées au sujet des objectifs immédiats-Qui sera chargé des activités? Quels éléments de quelle activité? quelles sont les personnes visées? quand? où et dans quel but?

Plus précisément, il est essentiel de répondre aux questions suivantes:

\section{A Qui sera chargé de l'exécution du programme d'intervention?}

Indiquer non seulement le ou les organismes qui seront chargé(s) de l'exécution, mais aussi les différentes catégories de personnel concernées (infirmières, médecins, sages-femmes, animatrices, etc.) ainsi que le rôle de chacun.

\section{B Où les activités liées à l'intervention auront-elles lieu?}

Donner le plus de détails possibles sur les zones concernées par le programme d'intervention. Ce programme concerne-t-il toute une région, un district, 10 villages, 30 usines, ou quatre cliniques? Bien mentionner les sites d'intervention.

\section{Quelles activités seront menées?}

Indiquer l'ordre chronologique des différents types d'activité. Par exemple, une intervention peut démarrer par la formation du personnel de terrain. La nature et la durée de la formation doivent être spécifiées. Ensuite, une série de réunions au niveau du village doit être organisée. Spécifier la fréquence et l'objectif de ces réunions. Enfin, il est prévu que le personnel visitera au moins cinq domiciles par jour pendant un an. Le but et la nature de ces visites doivent être mentionnés. Lors de la description des activités prévues, il est important d'en indiquer le niveau et l'intensité; par exemple, s'il s'agit d' une intervention dans le cadre d'une campagne IEC, cette dernière doit être décrite d'une manière complète. Indiquer le type de média qui sera utilisé (la télévision, la radio, la presse, les prospectus, les affiches, les réunions de groupes, les pièces de théâtre ou les chansons); mentionner le degré d'intensité de cette campagne 1100 affiches, 1000 prospectus, trois flash publicitaires radiophoniques par jour pendant six mois, un programme télévisé par semaine pendant trois mois); préciser qui sera chargé de la production des affiches, des prospectus, des scripts radiophoniques et télévisés, des pièces et des chansons et, si nécessaire, quel type de formation et d'équipement seront requis. En d'autres termes, fournir des informations précises et détaillées. 


\section{Exemple de description détaillée d'une étude: Afrique}

Une étude de recherche opérationnelle expérimentale en Gambie pour la prestation des services de planification familiale sur les lieux de travail.

\section{Sites d'intervention}

Cette étude visant la mise en place de services de planification familiale sur les lieux de travail sera conduite par l'Association Gambienne pour la Planification Familiale (GFPA). Tous les sites concernés seront situés à Banjoul ou ses environs. Les sites potentiels devront comprendre au moins 100 employés dont $20 \%$ seront des femmes. On estime à 25 le nombre de sites répondant à ces critères et à 15-20 le nombre d'entreprises qui accepteront de participer au projet. Ainsi, au minimum, 15 sites de travail devraient compter environ 1500 employés $(15$ x 100 employés $)$ parmi lesquels $300(1500 \times 0,20)$ devraient être des femmes. En fait, il est probable que le nombre total d'employés et de femmes soit plus élevé.

Bien qu'il n'y ait pas en Gambie de grandes entreprises industrielles, certaines d'entre elles telles que les brasseries, les hôtels, le port et les conserveries emploient plus de 100 ouvriers. Il y a dans quelques unes d'entre elles une majorité d'employées. Par exemple, une usine de conditionnement de crustacés emploie plus de 100 femmes. Le GFPA a déjà contacté un certain nombre d'entreprises afin de déterminer leur intérêt éventuel pour une participation à l'étude, et toutes ont donné des réponses positives.

Avant le début de l'intervention, des visites sur les sites d'intervention ainsi que des séminaires en direction des responsables et des représentants des travailleurs seront organisés par le GFPA afin d'assurer leur participation active et d'éclairer l'étude. Le but de ces séminaires sera de donner un aperçu des grandes lignes et objectifs du projet ainsi que de convaincre les responsables des avantages à long et à court terme dont ils bénéficieront grâce à leur participation. Ces séminaires seront organisés en collaboration avec le Ministère du Travail et de la Santé.

\section{Plan d'action}

Les entreprises ayant donné leur accord pour participer au projet seront classées selon le nombre de femmes qui y sont employées. Il y aura deux catégories: 1) les entreprises qui emploient de 20 à 49 femmes, et 2) les entreprises qui en emploient 50 et plus. Ces entreprises ainsi classées seront réparties d'une manière aléatoire sur trois groupes-les services de prestation fixes, les services de prestation mobiles et le groupe témoin. Les activités à mener pour les deux groupes expérimentaux sont les suivantes:

\section{a Prestations de services fixes}

Un distributeur de services de planification familiale pour 40 travailleurs sera choisi par les travailleurs eux-mêmes dans le site de prestation de services fixe, avec l'accord de la direction. Ce distributeur recevra une formation de 10 jours au total sur la planification familiale et les maladies sexuellement transmises (MST). Cinq jours de formation auront été effectués avant le commencement de la phase d'intervention de l'étude. Les cinq jours restants seront répartis durant la durée de l'étude à raison d'un jour à chaque fois.

Le distributeur de services sera chargé essentiellement de fournir à ses collègues les informations nécessaires sur la planification familiale et les MST, de vendre les condoms et les spermicides moyennant un bénéfice, et d'orienter les femmes vers les cliniques du GFPA pour les méthodes de contraception telles que le DIU et la stérilisation. Chaque distributeur recevra trois T-shirts aux couleurs vives frappés du logo du GFPA, ce qui aidera les travailleurs à les reconnaître. Ils recevront aussi une mallette de rangement pour les contraceptifs ainsi que des fiches de statistiques de services.

Les sites de prestation de services fixes seront visités une fois par mois par une éducatrice du GFPA qui est aussi une sage-femme. Cette éducatrice/sage-femme sera chargée essentiellement de vendre au distributeur les condoms et spermicides destinés à être revendus à la clientèle. Le distributeur pourra garder la moitié du prix de vente final. Actuellement, un sachet de quatre condoms se vend 12 cents. Ainsi, pour chaque sachet de quatre condoms, le distributeur gagnera 6 cents et le GFPA sera remboursé de 6 cents. 
L'éducatrice/sage-femme du GFPA examinera les femmes qui désirent prendre la pilule; elle fournira aux femmes aptes à utiliser cette méthode un premier cycle et les ré-approvisionnera lors de ses visites suivantes. Alors que les pilules seront fournies gratuitement à la clientèle, l'éducatrice/sage-femme fera payer à chaque utilisatrice une petite somme forfaitaire pour frais de services comme cela se fait dans les cliniques GFPA.

A part le fait que l'éducatrice/sage-femme sera chargée de réapprovisionner le distributeur et de fournir les pilules aux anciennes et nouvelles utilisatrices, elle sera aussi chargée de tenir des réunions de groupes avec les employélels pour discuter des MST, des méthodes de contraception, de leurs effets secondaires et des points d'approvisionnement. On demandera aux responsables concernés d'autoriser leurs employé(e)s à prendre 15 minutes sur leur temps de travail toutes les deux semaines pour assister à des séances d'éducation. On demandera aussi aux employé(e)s de prendre sur leur pause un temps égal pour ces mêmes sessions d'éducation.

\section{b Sites de prestations de services mobiles}

Les entreprises faisant partie du groupe prestation de services mobile seront visitées toutes les deux semaines par une éducatrice/sage-femme qui tiendra des réunions de groupe pour discuter avec les employé(e)s des méthodes de contraception, de leurs effets secondaires et des points d'approvisionnement en contraceptifs. On demandera aux responsables d'accorder à leurs employé(e)s 15 minutes sur leur temps de travail pour des séances d'éducation toutes les deux semaines. On demandera aussi aux employé(e)s de donner 15 minutes sur leur temps de pause pour ces séances d'éducation qui aborderont aussi le sujet des MST, y compris le SIDA.

Par ailleurs, l'éducatrice/sage-femme vendra des condoms et des spermicides à la clientèle. Le prix de vente reviendra entièrement à la GFPA. Comme pour les sites de prestation de services fixe, les pilules seront fournies gratuitement mais une petite somme forfaitaire pour frais de service sera prélevée. En ce qui concerne les autres méthodes comme le DIU et la stérilisation, l'éducatrice/sage-femme prendra des rendez-vous pour les employées dans les cliniques GFPA les plus proches.

\section{Recrutement et formation du personnel de terrain}

Le GFPA comprend déjà un certain nombre d'éducatrices/sage-femmes chargées d'exécuter les programmes d'information, d'éducation et de communication de l'Association. Quatre d'entre elles seront choisies pour travailler sur cette étude de recherche opérationnelle. Leur tâche consistera à conduire les activités sur le terrain, à programmer les visites d'éducation sur place, à superviser la distribution des contraceptifs; elles seront chargées aussi de recueillir les statistiques de services sur les ventes et les utilisatrices nouvelles et anciennes; enfin, elles seront responsables de la formation et de l'éducation sur place des distributeurs d'entreprise. Deux éducatrices/sages-femmes seront responsables des activités du groupe de prestations de services fixes et deux autres seront responsables du groupe prestation de services mobile.

Bien que ces éducatrices/sage-femmes ont déjà été formées en matière de planification familiale, un programme d'orientation de courte durée sera mis en place pour elles. Ce programme d'orientation sera l'occasion de discuter du contenu du projet et de revoir les besoins et la répartition des responsabilités du personnel de terrain GFPA et des distributeurs d'entreprise.

Un cadre GFPA sera nommé assistant chercheur. Il sera chargé des interviews des employé(e)s et de la collecte des statistiques de service ainsi que des autres données recueillies dans les entreprises. La formation de l'assistant de recherche sera fourni par la GFPA.

Le recrutement des distributeurs d'entreprise se fera sous le contrôle du Chercheur Principal avec l'assistance des deux éducatrices/sage-femmes. Les employé(e)s de l'entreprise seront encouragé(e)s à participer au choix de leur distributeur. On leur fera connaitre les critères de selection pour les aider à identifier un distributeur répondant à ces critères. Les distributeurs choisis doivent se montrer interessés par le projet, avoir une attitude positive en ce qui concerne la contraception, et être respectés par les employé(e)s et par la direction.

Les distributeurs devront participer à une première session de formation d'une durée de cinq jours et à une série de sessions de formation d'un jour qui seront organisées par le personnel du GFPA. Ces journées de formation seront progammées à des dates qui conviennent le mieux aux participants et aux formateurs. 


\section{En résumé-description détaillée de I'intervention}

1 Décrire avec le plus de détails possibles le programme d'intervention.

2 Décrire les activités dans l'ordre chronologique.

3 S'assurer que la description de l'intervention réponde aux trois questions fondamentales:

a Qui sera chargé de l'exécution?

b Où l'intervention aura-t-elle lieu?

c Quelles activités seront conduites, à quel niveau et à quel degré d'intensité? 


\section{Définitions opérationnelles}

Il s'agit dans l'étape suivante, après avoir présenté les objectifs et les hypothèses de recherche, de définir d'une manière opérationnelle aussi bien les variables clés de l'étude que la terminologie qui sera utilisée. La définition opérationnelle a deux objectifs: 1) la présentation des méthodes et des règles que suivra le chercheur afin de mesurer les variables, et 2) la définition précise des termes qui seront employés, afin d'éviter toute équivoque. Tout projet de recherche doit comprendre la définition opérationnelle des principales variables et de la terminologie.

\section{A Définition opérationnelle des variables}

Prenons à titre d'exemple comme variable indépendante la connaissance en matière de planification familiale. Avant que cette variable ne puisse être mesurée, il est nécessaire d'indiquer la méthodologie précise adoptée pour mesurer cette variable ainsi que le sens donné à l'expression "connaissance" en matière de planification familiale. Cette variable sera définie par le biais de faits observables qui serviront comme indicateurs du phénomène "connaissance" en matière de planification familiale. En effet, le terme "connaissance" ne constitue pas en lui-même un fait observable puisqu'il ne peut être ni senti, ni touché, ni goûté, ni entendu; c'est ainsi que le chercheur aura recours en général à une série de questions dont les réponses serviront de faits observables, c'est-à-dire d'indicateurs de la "connaissance." L'enquête devra répondre, par exemple, aux questions suivantes: "Avez-vous déjà entendu parler de la pilule?"; "Pouvez-vous me dire combien de fois une femme doit prendre la pilule pour éviter d'être enceinte?"; "Avez-vous déjà entendu parler du DIU?". Chacune de ces questions constitue un indicateur d'un aspect relatif à la connaissance en matière de contraception. Toutes les questions et toutes les réponses représentent aussi des faits observables susceptibles d'être mesurés.

L'étude peut faire appel à vingt questions relatives à la connaissance. Pour chaque réponse positive, l'enquêteur enregistre un score de 1 (un). A l'inverse, pour chaque réponse négative, l'enquêteur enregistre un score de 0 (zéro). Le score final pour chaque enquête en matière de connaissance peut être obtenu en faisant la somme des réponses positives aux 20 questions. L'éventail des scores irait ainsi de zéro à 20. Les personnes ayant obtenu 20 seraient considérées comme celles ayant la meilleure connaissance en matière de planification familiale. Et c'est ainsi que la variable “connaissance en matière de planification familiale" peut être définie d'une manière opérationnelle. Ce qui peut être présenté dans la proposition de recherche comme suit:

Connaissance en matière de planification familiale $=$ Nombre de réponses positives aux 20 questions relatives à la contraception.

Il est évident que ce qui précède ne constitue pas l'unique définition possible de la variable. On pourrait, si l'on veut, classer les niveaux de connaissance selon des catégories différentes en distinguant entre les niveaux élevé, moyen, faible ou nul des enquêtes en matière de planification familiale. Ces niveaux correspondent à des catégories (ou classes) de la variable et le classement des réponses dans l'une ou l'autre des catégories doit obéir à une règle adoptée à cette fin. Soit à titre d'exemple: 
Niveau élevé de connaissance $=5$ réponses positives ou plus sur les 20 questions.

Niveau moyen de connaissance $=$ Minimum de 8 réponses positives et maximum de 14 sur les 20 questions.

Niveau nul de connaissance $=$ Aucune réponse positive sur les 20 questions.

Il est à remarquer que les quatre classes ne se chevauchent pas et s'excluent mutuellement. De ce fait, la règle de classement adoptée ne permet pas à une personne classée dans la catégorie "niveau élevé de connaissance" d'être classée en même temps dans l'une ou l'autre des autres catégories. Par ailleurs, il n'existe pas de cinquième ou sixième classes, vu que ces quatre catégories incluent tous les niveaux de connaissance possibles.

Il est parfois préférable de ne pas être aussi précis dans la définition préalable des catégories; et il vaut mieux dans certains cas fixer les bornes limitant chaque catégorie seulement après la collecte des données et l'examen de la distribution de la variable. En règle générale, on devrait classer les réponses de manière à obtenir des catégories ayant approximativement les mêmes effectifs. Ainsi, dans l'exemple précédent, chacune des quatre classes devrait inclure 25 pourcent environ des réponses. Dans le cas où il serait nécessaire d'examiner la distribution de la variable avant de fixer les différentes catégories de réponse, il faudrait alors indiquer dans le chapitre des définitions opérationnelles les intitulés des différentes catégories, tout en spécifiant qu'elles comprendront des effectifs approximativement égaux.

Chaque variable doit avoir au moins deux catégories de réponses, sinon ce serait une constante. Il est toujours préférable dans la définition opérationnelle d'une variable de diviser celle-ci en un nombre assez important de catégories. Dans les exemples ci-dessus, la variable "connaissance en matière de planification familiale" est mesurée selon un éventail allant de zéro à 20 totalisant 21 catégories différentes qui sont regroupées dans le second exemple, en un nombre plus réduit de catégories, soit quatre niveaux différents de connaissance à savoir-élevé, moyen, faible et nul. On peut réduire encore plus le nombre de classes et passer de quatre à deux catégories seulement:

$$
\begin{aligned}
\text { Connaissance de la planification familiale }= & \begin{array}{l}
\text { Au moins une réponse positive à l'une des } 20 \\
\text { questions. }
\end{array} \\
\text { Ignorance de la planification familiale }= & \begin{array}{l}
\text { Aucune réponse positive à l'une des } 20 \\
\text { questions. }
\end{array}
\end{aligned}
$$

Il est toujours possible, en partant de plusieurs catégories, de les regrouper ensuite en un nombre plus réduit mais l'inverse est impossible; en effet, si on commet l'erreur de partir d'un nombre relativement réduit de catégories, il est exclu d'en élargir ensuite l'éventail. En général, le regroupement des réponses, et le passage d'un nombre élevé de catégories à un nombre plus limité se fait une fois que l'opération de collecte des données est terminée et que l'analyse des fréquences de la variable est rendue possible.

\section{Exemples de définitions opérationnelles des variables}

$$
\begin{aligned}
\text { Acceptation du planification familiale }= & \begin{array}{l}
\text { Première utilisation de l'une des méthodes } \\
\text { modernes de contraception. }
\end{array} \\
\text { Performance du personnel de terrain }= & \begin{array}{l}
\text { Nombre de tâches exécutées par le } \\
\text { personnel sur un nombre total de } 20 \text { tâches } \\
\text { préalablement établies. }
\end{array}
\end{aligned}
$$

Attitude vis-à-vis de la planification familiale $=$ Le nombre de réponses positives à 10 questions indiquant une attitude favorable à l'utilisation de la contraception.

\section{B Définitions opérationnelles de la terminologie}

Rappelons qu'une hypothèse de recherche exprime la relation probable existant entre deux ou plusieurs variables. Comme il a fallu fournir des définitions operationnelles des variables, il faudra 
aussi fournir des définitions opérationnelles de la terminologie décrivant les relations entre les variables. A titre d'exemple, on peut trouver dans la formulation des hypothèses des termes comme ce qui suit:

\begin{tabular}{ll}
$\begin{array}{ll}\text { supérieur à } \\
\text { inférieur à } \\
\text { plus élevé que } \\
\text { plus bas que }\end{array}$ & $\begin{array}{l}\text { plus grand que } \\
\text { plus fort que } \\
\text { plus important que } \\
\text { plus petit que }\end{array}$ \\
\multicolumn{2}{l}{ ou encore comme : } \\
$\begin{array}{ll}\text { plus sur } & \text { significatif } \\
\text { satisfaisant } & \text { répandu } \\
\text { meilleur } & \text { augmenté }\end{array}$
\end{tabular}

Chacun de ces termes peut avoir des significations différentes et il est donc nécessaire de fournir une définition opérationnelle pour chacune d'entre eux. La difficulté avec des termes comme plus que, augmenté, c'est qu'ils expriment une comparaison sans indiquer pour autant l'importance de la variation. On a besoin de savoir de combien est-ce supérieur à quoi et augmenté de combien.

Soit à titre d'exemple, l'hypothèse de recherche suivante:

Un programme de cinq semaines de formation pratique de terrain fera augmenter le degré de connaissance en matière de santé et de planification familiale chez le personnel qui a participé à ce programme.

Dans cette hypothèse, le programme de formation constitue la variable indépendante qui est déjà définie, au moins en partie, par une durée de cinq semaines et par le fait qu'il s'agit d'une formation pratique de terrain. La connaissance en matière de planification familiale constitue la variable dépendante qui a déjà été définie comme le nombre de réponses positives à 20 questions. Il reste à définir le terme augmenter, sinon il ne sera pas possible de connaître à partir de quel niveau l'hypothèse de recherche serait vérifiée ou non. En d'autres termes, on a besoin d'une échelle de référence qui puisse montrer l'importance de l'augmentation. Ainsi, on peut définir le terme augmenter comme suit:

$$
\begin{aligned}
\text { Augmentation }= & \text { Le score moyen de connaissance de la planification familiale obtenu par } \\
& \text { les participants à la formation est supérieur et significativement différent } \\
& \text { de celui obtenu par un groupe témoin n'ayant pas reçu cette formation. }
\end{aligned}
$$

Il est à noter que dans cette définition opérationnelle, on n'explique pas seulement le sens donné à augmentation, mais on fournit en même temps la procédure adoptée pour mesurer l'augmentation. Le score moyen de connaissance de planification familiale obtenu par les participants à la formation (groupe expérimental) sera comparé au score moyen obtenu par un groupe témoin. L'hypothèse de recherche ne sera vérifiée que si le score moyen des participants à la formation est supérieur et significativement différent du score moyen obtenu par le groupe témoin. Enfin, pour être tout à fait clair, il convient de définir ce qu'on entend par le terme significativement.

$$
\begin{aligned}
& \text { Significativement }=\text { Le score moyen obtenu par les participants est supérieur à celui du } \\
& \text { groupe témoin avec une probabilité de 0,95. }
\end{aligned}
$$

En resumé, à la rubrique définitions opérationnelles, le chercheur doit présenter les méthodes et les règles qu'il utilisera pour mesurer les variables ainsi que la signification des termes employés. Ceci devra être fait au moyen d'indicateurs d'évènements observables. On doit pouvoir poser une question, écouter une réponse, observer un comportement, enregistrer un fait, mesurer une caractéristique. Les variables pourront être divisées en catégories qui s'excluent mutuellement et qui incluent toutes les éventualités possibles. Dans les définitions opérationnelles, le chercheur présentera enfin une échelle de référence qui lui permettra de vérifier ou de rejeter par la suite l'hypothèse de recherche. 


\section{Exemples de définitions opérationnelles de la terminologie}

Utilisateur de planification familiale $=$ Toute femme mariée âgée de 15-44 ans et tout homme marié âgé de plus de 15 ans déclarant avoir utilisé au moins une fois une méthode moderne de contraception au cours du mois dernier.

Méthode moderne de contraception $=$ L'une des méthodes suivantes de prévention de grossesse: pilules, DIU, stérilisation féminine et masculine, Depo-Provera, condoms, diaphragmes.

Meilleure performance du personnel $=$ Tout membre du personnel qui recrute de nouveaux utilisateurs, tient plus de réunions ou fournit plus de sachets de réhydration qu'au cours des six mois précédents.

Village moderne $=$ Tout village pourvu de trois ou plus des équipements suivants: électricité, dispensaire public, route goudronnée d'environ $1 \mathrm{~km}$, école primaire, banque, bureau de poste, un système d'irrigation couvrant 50 pourcent ou plus des terres cultivées.

\section{En résumé-présentation des définitions opérationnelles}

1 Rédiger une définition opérationnelle pour chacune des variables dépendantes ou indépendantes.

2 Rédiger une définition opérationnelle pour chacun des termes utilisés pour décrire la relation entre ces variables.

3 Pour chaque définition, se demander:

a les règles et méthodes pour mesurer la variable sont-elles clairement formulées?

b les catégories, mutuellement exclusives et incluant toutes les éventualités possibles, ont-elles été bien spécifiées? 


\section{Procédés de recherche}

\section{A Des données fiables et justes}

Il s'agit ici de décrire le procédé de recherche choisi par le chercheur afin d'obtenir les résultats les plus fiables et les plus justes possibles, avec une marge d'erreur réduite à son minimum.

La fiabilité a trait à la cohérence, à la stabilité ou à la sécurité des données. Les données sont fiables si le chercheur, en recommençant une deuxième fois l'opération de collecte, obtient les mêmes données. $\mathrm{Si}$, à la deuxième collecte les réponses ne sont pas les mêmes, la mesure n'est pas fiable. Mais des données fiables n'impliquent pas forcément qu'elles soient justes. Des données justes sont non seulement fiables mais également exactes et précises. En d'autres termes, une mesure est juste si elle fait ce que l'on attend d'elle. Si une mesure est juste, elle est forcément fiable. Par contre, une mesure fiable n'est pas forcément juste. Par exemple, supposons qu'on demande à quelqu'un "quel âge avez-vous" et qu'il réponde "j'ai 49 ans". Supposons qu'on lui pose une deuxième question pour vérifier la cohérence de la réponse comme "En quel mois et en quelle année êtes-vous né?" et qu'il réponde "Septembre 1941". Si l'enquête se déroule en Février 1991, on voit que la personne interrogée a vraiment 49 ans.

Dans cet exemple, on a posé deux questions différentes pour mesurer l'âge et on a obtenu le même résultat. Les réponses sont ainsi cohérentes et fiables. Supposons maintenant que l'enquêteur regarde par hasard l'acte de naissance de la personne interrogée et qu'il trouve que la vraie date de naissance est Septembre 1938. Ainsi les données déjà obtenues, bien que fiables et cohérentes, sont inexactes et ne sont pas pour autant justes.

\section{B Sources d'erreurs}

L'une des critères qui détermine le procédé de recherche est le degré de maîtrise des facteurs qui constituent souvent des sources d'erreurs. En adoptant un type donné d'investigation, le chercheur désire obtenir des informations justes et précises en évitant les sources potentielles d'erreur qui sont assez nombreuses et dont les plus importantes sont les suivantes:

\section{Effet de conjoncture}

Parfois, pendant le déroulement d'un projet, des événements tendent à amplifier ou à diminuer l'effet attendu du projet. Ce sont des effets de conjoncture. Les événements survenus ne font pas partie du projet, ils n'étaient pas prévus et on ne s'y attendait pas. Ces événements se produisent et ont des effets sur les résultats de l'étude.

Supposons, par exemple, qu'un groupe d'agents de terrain ait suivi un stage de formation visant à améliorer leurs performances, et que, six mois plus tard les résultats d'une évaluation n'indiquent pas une amélioration de leurs performances. On pourrait conclure que le programme de formation a été un échec. Mais en procédant à un examen plus approfondi de la situation, on trouve que ces agents avaient décidé de faire grève pendant trois semaines, juste après le stage, pour des revendications salariales. La grève qui a eu lieu n'était pas prévue dans le cadre du projet et 
affecte les résultats sur les performances des agents. Elle constitue un phénomène de conjoncture. La question de la réussite ou de l'échec du stage de formation demeure toujours posée.

L'impact du stage de formation sur les performances des agents pourrait être évalué si on arrivait à contrôler l'effet propre de la grève. Toute la question est de savoir si avec le type d'investigation choisi, le chercheur peut arriver à effectuer cette évaluation.

Soit un autre exemple: Supposons qu'on veuille évaluer l'impact d'une campagne de motivation en matière de planification familiale sur les connaissances et les attitudes de la population. Un moyen de le faire consisterait à effectuer une enquête avant la campagne et une autre après, afin de comparer les résultats. Cette comparaison pourrait montrer que les connaissances de la population en matière de planification familiale se sont accrues et que les attitudes sont devenues plus favorables. Le chercheur peut conclure dans ce cas que la campagne de motivation a reussi et atteint ses objectifs. Mais supposons que le Président du pays ait fait, au cours de la campagne, une déclaration à la radio et à la télévision au sujet de l'accroissement rapide de la population et de l'utilité de la planification familiale. La question qui se pose alors est de savoir si l'amélioration des connaissances et des attitudes concernant la planification familiale est due à la campagne de motivation, ou à la déclaration présidentielle ou encore aux deux phénomènes à la fois. La déclaration présidentielle constitue, dans ce contexte, une donnée conjonctuelle. Dans cet exemple, l'effet propre de la campagne de motivation sur les connaissances et attitudes de la population en matière de planification familiale ne pourra pas être mesuré sauf si, dans le cadre du procédé de recherche choisi, certaines dispositions permettent de dégager les effets propres de la campagne de motivation d'une part et de la déclaration présidentielle d'autre part.

\section{Effet de sélection}

Il arrive souvent que les caractéristiques des personnes qui forment le groupe témoin soient différentes de celles des personnes qui constituent l'objet de l'étude, ce qui nuit à l'exactitude des résultats. Supposons que les habitants d'un groupe donné (groupe expérimental) bénéficient d'un programme de motivation pour la planification familiale et qu'un autre district (groupe témoin) n'en bénéficie pas. Une évaluation du programme de motivation est effectuée un an plus tard par comparaison des proportions de contraceptrices des deux districts. Cette évaluation montre que la proportion a significativement augmentée dans le district expérimental par rapport au district témoin, ce qui tendrait à démontrer le succès du programme de formation. Mais un examen plus approfondi de la situation montre que les caractéristiques des deux populations sont, en fait, très différentes. Les habitants du district expérimental sont en général plus jeunes, plus instruits, plus urbanisés et plutôt plus aisés que les habitants du district témoin; ils sont ainsi susceptibles d'adopter plus facilement la planification familiale avec ou sans programme spécial de motivation. La question de l'effet propre de ce programme reste donc toujours posée. La réponse aurait été possible si les caractéristiques des habitants des deux districts n'étaient pas aussi différentes. En raison de cette sélection, il n'est pas ainsi possible de distinguer l'effet exact du programme de formation de celui des différences de structure entre les deux districts.

\section{Effet de répétition}

Les résultats d'un test final qu'on effectue à la fin d'une étude peuvent être influencés par le pré-test effectué au démarrage d'un projet. Les réponses obtenues à un test final ont des chances d'être meilleures si les personnes interrogées (par exemple des stagiaires) ont déjà répondu aux questions posées au pré-test, dans la mesure où ces personnes peuvent se souvenir des questions et des erreurs commises. Ainsi, la meilleure performance observée au test final peut être due plutôt à l'effet du pré-test qu'au programme lui-même (par exemple, un stage de formation). Cette situation se produirait par exemple pour des enquêtes sur les connaissances, les attitudes et les pratiques en matière de contraception (C.A.P.) effectuées annuellement pendant une certaine période sur les mêmes personnes. Au bout d'un certain temps, les personnes interrogées arrivent à donner les réponses adéquates à des questions comme "Pouvez-vous nommer trois méthodes contraceptives modernes?", non pas sous l'effet d'un programme éducatif et donc d'une meilleure connaissance de la planification familiale, mais sous l'effet de leur expérience avec l'enquête C.A.P. Ainsi, l'effet de répétition entre en jeu lorsqu'on fait passer plusieurs fois le même test aux mêmes personnes. 


\section{Effet du support de collecte}

Un autre type d'effet qui peut se produire indépendamment du projet ou du programme résulte des modifications que subit l'outil de mesure (par exemple un questionnaire) entre le pré-test et le test final. Si, pour mesurer le degré de connaissance en matière de planification familiale par exemple, on pose la question "Avez-vous déjà entendu parler de la planification familiale?" pendant le prétest, et la question "Pouvez-vous citer trois méthodes modernes de contraception?" pendant le test final, il est probable qu'on aboutisse à des résultats différents; mais cette différence peut être due à la modification de la question. Le même type d'effet peut se produire lorsque les enquêteurs acquièrent plus d'expérience. Un enquêteur expérimenté peut obtenir des informations plus complètes qu'un enquêteur ayant moins d'expérience. La qualité meilleure des données ainsi obtenues peut être due non pas au programme mis en place, mais à une observation des faits et à une manière de poser les questions qualitativement meilleure. Ainsi, la modification du support de collecte des données peut avoir une influence sur la qualité de celles-ci.

\section{Effet de durée}

Certaines modifications des résultats entre le test final et le pré-test peuvent être dues au facteur temps. Les programmes de formation de longue durée peuvent connaître ce type d'effet. Ainsi, les stagiaires peuvent très bien arriver à la fin du stage relativement perturbés, que ce soit par la fatigue, l'ennui ou la faim. Dans d'autres cas, notamment dans les études longitudinales qui durent plusieurs années, les personnes peuvent devenir plus résistantes, acquérir plus de connaissance et plus d'expérience et, par conséquent, plus de maturité; ce qui peut provoquer des effets indépendants du programme mis en place. Ainsi, si les stagiaires pertubés par la fatigue, l'ennui ou la faim, passent un test, on peut s'attendre à obtenir en moyenne des résultats plus bas que ceux que l'on aurait obtenus si les stagiaires avaient été en meilleure forme. Un évaluateur qui compare ensuite ces résultats bas aux résultats de pré-test pourrait constater que la différence est très faible et conclure alors que le stage de formation est un échec. Mais est-ce vraiment le cas, ou bien les résultats sont-ils dus seulement à l'effet de durée? La durée peut introduire ses propres effets sur la qualité des données observées.

\section{Sortie du champ d'observation}

Dans les études prospectives cohortes ou de générations (appelées aussi études de panels) qui impliquent le suivi d'un même groupe d'individus dans le temps, certaines personnes peuvent disparaître du champ d'observation. Les personnes interrogées lors du premier passage ne seront jamais toutes retrouvées pour l'enquête du deuxième passage, ce qui constitue une perte d'individus pour le suivi. Les effets sont observés lorsque les individus ainsi perdus de vue possèdent des caractéristiques nettement différentes de celles des individus retrouvés. Les différences observées entre les résultats des deux passages successifs peuvent être dues en partie à l'effet de sortie plutôt qu'à l'effet du programme mis en place.

Il est important dans la conception de l'étude, d'examiner soigneusement les sources possibles d'erreur, effets de sélection, de répétition, de support, de durée et de sortie, autant de facteurs perturbateurs qui peuvent expliquer certaines différences observées. Il s'agit, en d'autres termes, de pouvoir distinguer les effets propres des programmes mis en place des effets introduits par les phénomènes perturbateurs.

\section{Procédés de recherche}

On présente, dans ce qui suit, les procédés les plus couramment adoptés dans les recherches opérationnelles en matière de planification familiale. Ce ne sont pas les seuls, mais ce sont les plus utilisés, que ce soit dans les études expérimentales, quasi-expérimentales ou non-expérimentales. Pour décrire ces procédés, on aura recours aux diagrammes et aux symboles préconisés par Campbell et Stanley (voir bibliographie) et qui sont couramment utilisés (figure 7.1).

Dans ce diagramme, les individus d'une même population sont répartis d'une manière 
FIGURE 7.1

Signification des diagrammes

et des symboles

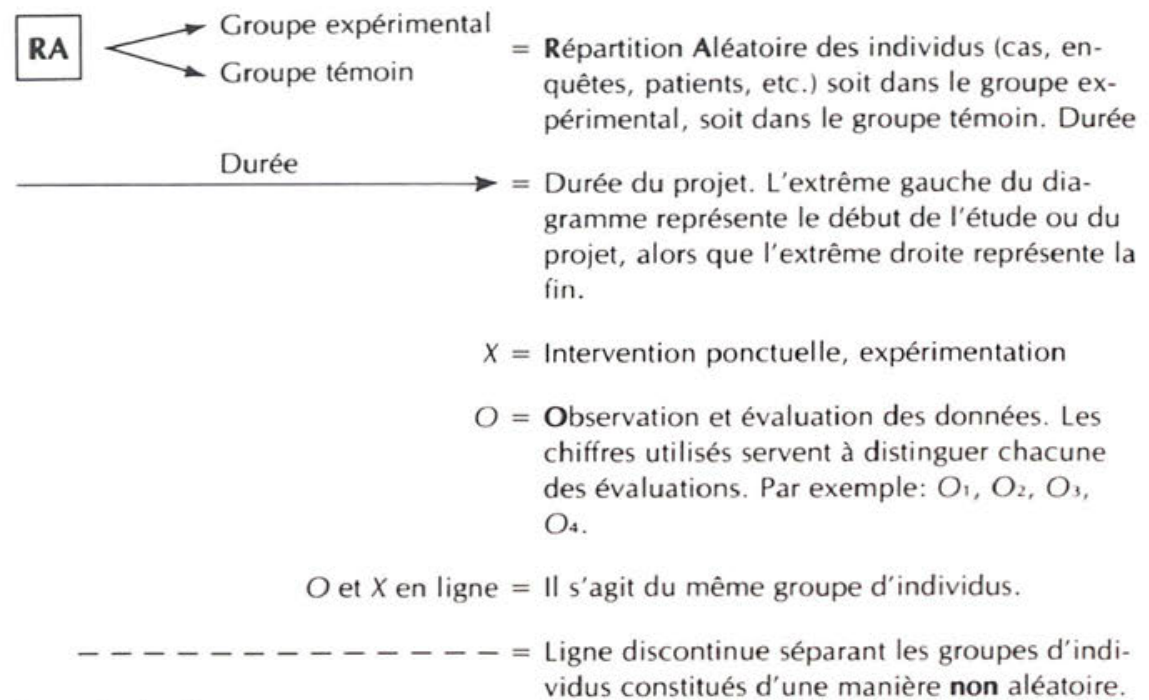

Exemple de diagramme vidus constitués d'une manière non aléatoire.
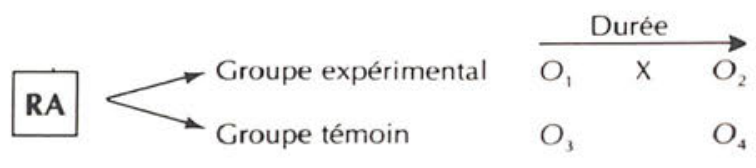

aléatoire (RA) dans chacun des groupes témoin ou expérimental. Chaque groupe fait l'objet d'une observation et d'une évaluation de départ (pré-tests $O_{1}$ et $O_{3}$ ). Le groupe expérimental fait ensuite l'objet d'un programme spécifique, à l'inverse du groupe témoin qui demeure tel quel. Chaque groupe fait l'objet, enfin, d'une observation et d'une évaluation $\mathrm{CO}_{2}$ et $\left.\mathrm{O}_{4}\right)$. Etant donné le programme spécifique subi par le groupe expérimental, on doit s'attendre a ce que $\mathrm{O}_{2}$ soit plus grand que $\mathrm{O}_{4}$. De même, puisque les individus ont été répartis au hasard dans chacun des deux groupes, on doit s'attendre à ce que $O_{1}$ soit égal à $O_{3}$ en ce qui concerne les variables principales comme l'âge, le sexe, la parité et l'instruction.

Le procédé de recherche décrit ci-dessus constitue une approche expérimentale typique puisqu'il permet d'éviter au maximum les risques d'erreur. Les procédés suivants seront décrits par référence à cette approche.

\section{Procédés non-expérimentaux}

Il éxiste différents procédés non-expérimentaux couramment utilisés par les chercheurs en matière de planification familiale. Ces procédés sont particulièrement adaptés aux études descriptives et aux études de cas. Par contre, il est préférable de ne pas y recourir pour évaluer l'impact d'un programme spécifique. Ces procédés sont utiles pour effectuer des diagnostics sur les causes possibles d'un problème.

\section{a Test final seulement}

$$
\text { Groupe expérimental } \stackrel{\text { Durée }}{\stackrel{X O_{1}}{\longrightarrow}}
$$

Dans cette approche on effectue une observation et une évaluation des données $\left(O_{1}\right)$ quelque temps après l'introduction d'un programme spécifique; ce qui ne peut fournir qu'une description de la situation puisqu'en raison de l'absence d'un groupe témoin, il n'est pas possible de comparer l'évaluation $\left(O_{1}\right)$ avec une autre. Les différents aspects tels que la conjoncture, la sélection, la durée et la sortie du champ d'observation constituent des sources d'erreurs possibles et non contrôlables dont il s'agit de tenir compte. Cette approche est utile dans une recherche qui a pour objectif, par exemple, de connaître les caractéristiques démographiques des contraceptrices ayant opté pour le DIU ou la stérilisation. L'adoption de l'une de ces méthodes de planification familiale est représentée 
par $(X)$ alors que les caractéristiques démographiques observées sont représentées par $O_{1}$. Cette approche peut fournir sur le programme des renseignements fort utiles moyennant le recours aux techniques d'analyse multivariée (voir chapitre 10 section B.2.f). Mais la comparabilité des résultats demeure néanmoins assez limitée.

\section{b Pré-test et test final}

Groupe expérimental $\stackrel{\text { Durée }}{\stackrel{O_{1}}{\longrightarrow} \times \mathrm{O}_{2}}$

On n'a pas recours ici non plus à un groupe témoin. L'évaluation dans le temps est fournie par une observation initiale $O_{1}$. On doit tenir compte dans cette approche, des sources d'erreurs possibles telles que la conjoncture, la répétition, la durée du projet et les modifications du projet de collecte des données.

\section{c Groupe témoin}

\begin{tabular}{cc}
\multirow{2}{*}{ Groupe expérimental } & Durée \\
\cline { 2 - 3 } Groupe témoin & $-\mathrm{O}_{1}$ \\
\hline & $\mathrm{O}_{2}$
\end{tabular}

Contrairement aux conceptions précédentes, cette approche utilise un groupe témoin ou de comparaison. On effectue une observation $\left(O_{1}\right)$ sur le groupe expérimental quelque temps après l'introduction d'un nouvel élément $(X)$. Les données sont ensuite comparées à celles obtenues à partir du groupe témoin $\left.\mathrm{C}_{2}\right)$ qui est resté tel quel. Comme on le voit, les deux groupes sont séparés par une ligne discontinue, ce qui signifie que la répartition des individus entre les deux groupes n'a pas été effectuée d'une manière aléatoire. On peut utiliser cette approche pour mesurer par exemple l'évolution intervenue chez les patients d'une clinique après l'introduction d'un élément spécial, par comparaison avec la situation d'une autre clinique qui n'a pas subi le même phénomène. Les sources d'erreur principales sont constituées, dans ce cas, par les facteurs dus à la sélection et à la sortie du champ d'observation. Ainsi, les caractéristiques relatives à l'âge, à la parité et à l'instruction des deux groupes peuvent être fort différentes au départ. En plus, la sortie du champ d'observation d'un certain nombre d'individus du groupe expérimental ayant subi le programme $(X)$ est toujours possible, ce qui les empêche de figurer au niveau de l'observation $O_{1}$. Certaines différences entre $O_{1}$ et $\mathrm{O}_{2}$ peuvent donc très bien provenir des effets de sélection et de sortie du champ d'observation, plutôt que des effets du programme $(X)$.

\section{Procédés expérimentaux}

\section{a Pré-test et test final avec un groupe témoin}

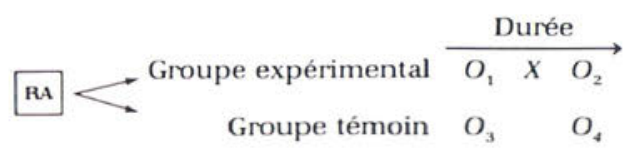

Ce procédé typiquement expérimental a déjà été discuté plus haut. Il se caractérise principalement par une répartition aléatoire des individus entre le groupe témoin et le groupe expérimental, ce qui donne des situations de départ identiques. Ainsi, les modifications de situation pourront être mesurées en termes probabilistes. Toute différence qui sera constatée entre les observations $\mathrm{O}_{2}$ et $\mathrm{O}_{4}$ sera attribuée aux effets du programme $(X)$. C'est cette approche qui permet de contrôler le mieux les effets des phénomènes perturbateurs et les sources d'erreur possible. Cette conception théorique est toutefois difficilement réalisable sur le terrain. Il est, en effet, souvent impossible de répartir les individus d'une manière aléatoire entre les deux groupes. Pour des raisons morales ou pratiques, il est difficile de refuser à une partie de la population plutôt qu'à une autre l'accès au programme. Cependant, il peut y avoir des cas où la répartition aléatoire est possible; lorsque, par exemple, la demande d'un type de service est plus élevée que la capacité d'approvisionnement. Une 
telle situation se produit lorsqu'un nouveau type de contraceptif comme le NORPLANT ${ }^{\circledR}$ est introduit. La capacité d'approvionnement peut être très limitée alors que la demande potentielle est très élevée. Une situation similaire se produit parfois lorsque le nombre de personnes ayant besoin d'une formation est supérieur au nombre de places disponibles. Dans ce cas, les "sujets expérimentaux" peuvent être selectionnés d'une manière aléatoire à partir du groupe des clientes potentielles du NORPLANT ${ }^{\circledR}$ ou bien à partir du groupe des personnes à former. Les personnes qui n'ont pas été selectionnées deviennent le groupe témoin.

\section{b Test final avec un groupe témoin}

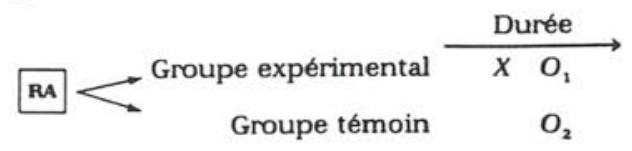

Cette approche constitue une autre variante des procédés expérimentaux. Elle diffère de la précédente par l'absence d'un pré-test. On suppose ainsi, grâce à la répartition aléatoire des individus entre les deux groupes, que les situations de départ sont identiques. La comparaison de la situation du groupe expérimental après l'introduction du programme avec celle du groupe témoin permet de mesurer les effets possibles du programme. Par contre, l'absence d'un pré-test ne permet pas de saisir l'ampleur du changement.

\section{Procédés quasi-expérimentaux}

En pratique, il est souvent très coûteux et assez difficile, voir impossible, de réaliser le critère principal de l'approche expérimentale qu'est la répartition aléatoire des individus. Il est pourtant souhaitable d'éviter les sources d'erreur possible avec les méthodes non-expérimentales. L'une des alternatives consiste à utiliser des procédés quasi-expérimentaux qui permettent au chercheur de se passer du critère de répartition aléatoire et de contrôler quand même certaines sources d'erreur possible.

\section{a Série chronologique}

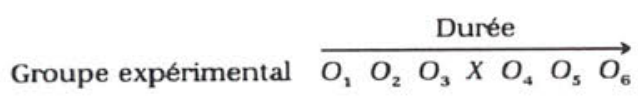

Cette approche ressemble au procédé non-expérimental utilisant un test final et un pré-test, avec l'avantage d'observations répétées en amont et en aval du programme $(X)$. Par exemple, si on ne décèle aucune variation entre $O_{1}, \mathrm{O}_{2}$ et $\mathrm{O}_{3}$, et qu'une brusque variation se produit entre $\mathrm{O}_{3}$ et $\mathrm{O}_{4}$ que l'on retrouve entre $\mathrm{O}_{4}$ et $\mathrm{O}_{5}$, on peut conclure avec beaucoup de certitude que c'est grâce aux effets du programme $(X)$. Ces variations peuvent être représentées par un graphique comme l'indique la figure 7.2.

Supposons que le programme introduit est constitué par une campagne d'éducation à grande échelle destinée a accroître, chez la population, le niveau de connaissance en matière de

FIGURE 7.2

Variation soudaine de la variable après l'introduction d'un programme

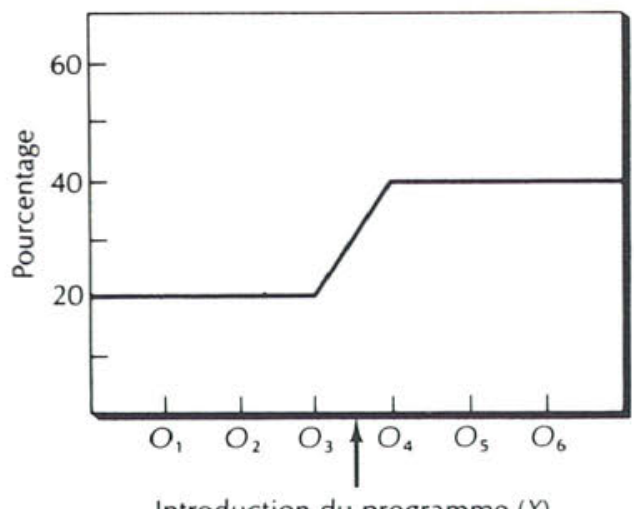


FIGURE 7.3

Même rythme de variation

d'une variable avant et après

l'introduction d'un

programme

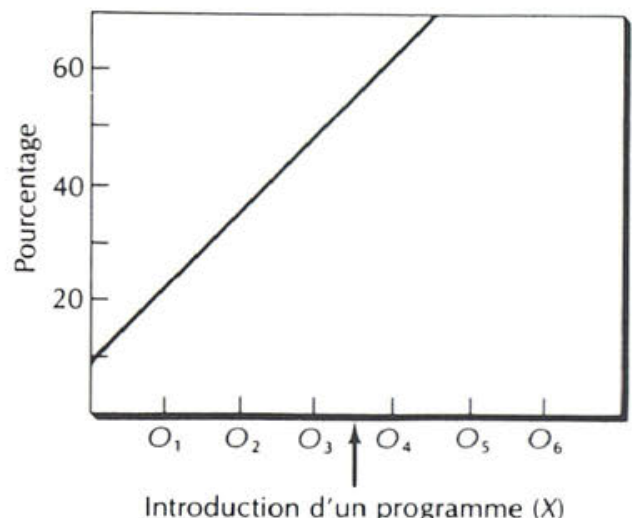

Introduction d'un programme $(X)$

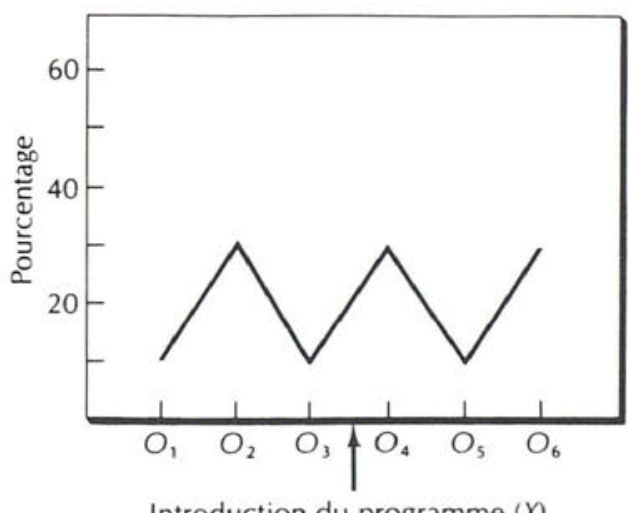

Introduction du programme $(X)$
FIGURE 7.4

Variation régulière avant et après l'introduction du programme

planification familiale. Les observations $\mathrm{O}_{1}, \mathrm{O}_{2}, \mathrm{O}_{3}$ montrent qu'avant l'introduction du programme, le niveau de connaissance de la planification familiale parmi la population adulte dans une région donnée se situe à 30 pourcent uniquement. Les observations $O_{4}, O_{5}$, et $O_{6}$ effectuées après l'introduction du programme montrent, par contre, que ce niveau grimpe à 50 pourcent. L'explication la plus vraisemblable de cette évolution est fournie par l'introduction de la campagne d'éducation. Mais l'existence d'un évènement conjoncturel entre $\mathrm{O}_{3}$ et $\mathrm{O}_{4}$ pourrait aussi expliquer cette évolution, ce qui constitue une source d'erreur.

Malgré les effets possibles des facteurs conjoncturels ou des facteurs liés à l'outil de mesure, la série chronologique permet au chercheur d'effectuer un diagnostic sérieux de la situation en évitant les grands risques d'erreur, comme on peut le constater dans la figure 7.3.

Ainsi, les pourcentages représentés dans ce graphique sont en augmentation régulière et continuent dans le temps. Le rythme d'augmentation n'a pas varié entre $\mathrm{O}_{3}$ et $\mathrm{O}_{4}$ après l'introduction du programme $(X)$. Ce programme n'a pas modifié, en fait, la tendance générale degagée par la série d'observations, d'où l'intérêt de la série chronologique qui peut fournir une description en termes de tendance. Si le chercheur avait eu recours au procédé utilisant un pré-test et un test final plutôt qu'à la série chronologique, il aurait conclu que l'introduction du programme a eu un effet positif sur l'évolution des pourcentages dans le temps, ce qui n'est en fait pas le cas.

Prenons un autre exemple, comme l'indique la figure 7.4. La figure 7.4 montre une alternance régulière et systématique de la hausse et de la baisse des pourcentages. Il ne semble pas que l'introduction du programme ait changé quoi que ce soit à cette tendance. On aurait pu conclure le contraire si on avait comparé $\mathrm{O}_{3}$ et $\mathrm{O}_{4}$ en ayant eu recours à un procédé utilisant un pré-test et un test final.

Le procédé utilisant les séries chronologiques convient lorsqu'on a accès à un système régulier de collecte des données telles que les statistiques mensuelles de services. Les données ainsi que le moment d'introduction du programme, peuvent être représentées sur un graphique. Si le tracé de la courbe montre un changement brusque qui s'écarte de la tendance générale, avant ou 

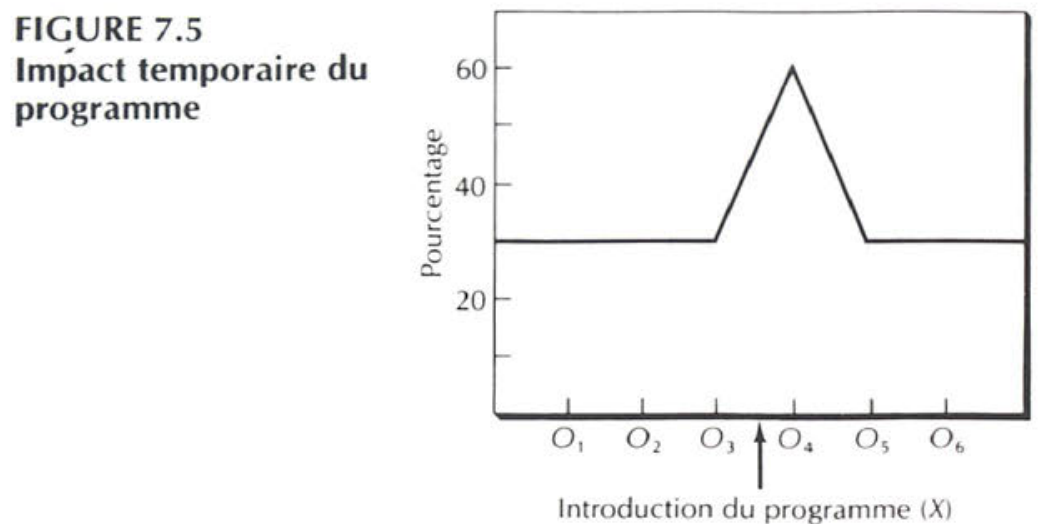

après l'introduction du programme, on peut conclure avec assez de certitude à l'existence d'un effet du programme.

Prenons l'exemple indiqué dans la figure 7.5. On suppose, dans cet exemple que la stérilisation est adoptée par 30 pourcent des nouvelles contraceptrices. Le chiffre est confirmé par toutes les observations $O_{1}, O_{2}$, et $O_{3}$. Une vaste campagne d'information et d'éducation est ensuite menée. L'observation $\mathrm{O}_{4}$ montre que le pourcentage de femmes ayant eu recours à la stérilisation passe à 60 pourcent. Enfin, quelque temps après la campagne, le pourcentage redescend à 30 pourcent. Ainsi, la campagne menée a eu une influence positive mais cette influence n'a pas duré. Dans ce cas aussi, le recours à un procédé utilisant un pré-test et un test final aurait permis de penser à un succès énorme de la campagne d'information et d'éducation par la comparaison des observations $\mathrm{O}_{3}$ et $\mathrm{O}_{4}$ seulement. Le caractère provisoire de cette influence n'aurait pas été détecté.

En résumé, malgré l'absence d'un groupe témoin et malgré les effets possibles des phénomènes perturbateurs tels que les évènements conjoncturels et les facteurs liés à l'outil de mesure, la série chronologique permet mieux l'analyse des données et le diagnostic de l'effet du programme que l'approche utilisant un pré-test et un test final. Les tendances observées en amont et en aval de l'introduction du programme sont dans ce cas d'une grande utilité. Cette approche est particulièrement indiquée lorsque plusieurs observations sont possibles aussi bien avant l'introduction du programme qu'après.

\section{b Groupe témoin non identique}

$$
\begin{array}{rc}
\multirow{4}{*}{\text { Groupe expérimental }} & \text { Durée } \\
\cline { 2 - 2 } \text { Groupe témoin non identique } & \mathrm{O}_{1} \times \mathrm{O}_{2} \\
\hline \mathrm{O}_{3} & \mathrm{O}_{4}
\end{array}
$$

En général, il est possible de confronter les données observées sur le groupe expérimental à celles observées sur un groupe témoin présentant certaines caractéristiques différentes. Supposons, par exemple, qu'on veuille évaluer les effets d'un programme de formation. Des groupes de 20 stagiaires peuvent suivre ce programme $(X)$ organisé par le centre de formation avec un pré-test préalable $\left(O_{1}\right)$ et un test final $\left(\mathrm{O}_{2}\right)$. Les données obtenues peuvent être confrontées à celles observées sur un groupe d'agents qui n'a pas encore suivi ce stage de formation mais ayant subi simultanément le même pré-test $\left(\mathrm{O}_{3}\right)$ et le même test final $\left(\mathrm{O}_{4}\right)$. Les renseignements obtenus lors des deux pré-tests $\left(\mathrm{O}_{1}\right.$ et $\left.\mathrm{O}_{3}\right)$ peuvent servir, dans ce cas, à mesurer le degré de similitude des caractéristiques des deux groupes, ce qui permet d'en tenir compte dans la comparaison des données observées en $\mathrm{O}_{2}$ et $\mathrm{O}_{4}$. On s'attend, dans ce cas, à un effet positif du programme $(X)$ entraînant une supériorité de $\mathrm{O}_{2}$ sur $\mathrm{O}_{4}$.

Cette approche s'adapte bien à l'évaluation des stages de formation, ou des effets d'un programme spécifique introduit dans une zône géographique donnée (un district par exemple). Dans ce dernier cas, les données observées peuvent être comparées à celles d'une zône limitrophe n'ayant pas bénéficiée de ce programme et pouvant présenter certaines caractéristiques différentes. Il est primordial, dans cette approche, de bien analyser les données fournies par les pré-tests $\left(O_{1}\right.$ et 
$\mathrm{O}_{3}$ ) et de détecter les effets possibles de la sélection et des caractéristiques différentielles du groupe expérimental et du groupe témoin, afin d'en tenir compte dans l'analyse du résultat final (comparaison $\mathrm{O}_{2}$ et $\mathrm{O}_{4}$ ).

\section{c Echantillons indépendants pour le pré-test et pour le test}

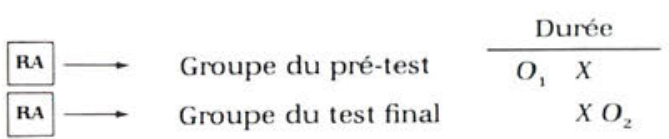

Cette conception est couramment utilisée dans les recherches en matière de planification familiale. On effectue un pré-test $\left(O_{1}\right)$ sur un échantillon aléatoire représentatif de la même population qui va bénéficier d'un programme $(X)$. Quelque temps après l'introduction du programme, on effectue un test final $\left.\mathrm{O}_{2}\right)$ sur un deuxième échantillon aléatoire pris au sein de la même population. Ce système permet d'éviter les effets de repétition mais non pas les effets liés à la conjoncture, à la durée du projet, à la sortie du champs d'observation ou encore aux modifications de l'outil de mesure.

\section{Choix d'un procédé de recherche}

Le choix d'un procédé de recherche est parfois une opération complexe qui implique toujours de prendre en considération les aspects éthiques, les nécessités techniques et les contraintes pratiques et administratives.

1 Aspects éthiques. L'aspect éthique est le premier à prendre en considération lorsqu'on choisit un procédé de recherche. Si un procédé de recherche conduit à des mesures contraires à l'éthique, à une violation des droits et de la dignité des personnes ou bien à une dénégation des services qui autrement seraient disponibles, il faut alors modifier, sinon abandonner ce procédé quelqu'en soient les conséquences sur la fiabilité, la validité, la durée de l'étude, les fonds financiers et le personnel engagé. En fait, s'il n'est pas possible de faire une étude répondant aux critères éthiques, cette étude ne doit pas être faite. Aucun compromis ne peut être fait sur ce point.

2 Contraintes pratiques et administratives. Tout chercheur rêve d'avoir pour son étude une grande disponibilité de fonds financiers, de temps, de personnel et de materiel (le dernier ordinateur, une belle imprimante laser, et peut-être un véhicule tout terrain bien confortable). Malheureusement, les rêves sont loin de la réalité. La plupart du temps, les fonds suffisent à peine, le temps est compté, le personnel peu nombreux et les équipements dépassés ou inexistants. Il est évident que ces conditions bien réelles affectent directement la recherche et en particulier, le procédé de recherche. Il se peut que les fonds, le temps et le personnel disponibles ne permettent pas d'envisager trois groupes expérimentaux et un groupe témoin ni une enquête de grande envergure très complète suivie d'une étude de suivi 24 mois après. Il se peut aussi que les ressources existantes ne permettent pas de conduire une intervention durant 36 mois. Toutes ces contraintes pratiques et administratives ne peuvent qu'avoir une influence sur le choix du procédé de recherche.

3 Aspects techniques. Alors que l'objectif sur le plan technique est de choisir un procédé de recherche à même de minimiser les erreurs possibles, la variabilité du terrain où se déroule la recherche opérationnelle fait que presque toujours, la fiabilité et la validité de l'étude peuvent être affectés. Toute étude rencontre des difficultés dues à des évènements imprévus tels que la fermeture d'un centre de prestation de services, la mutation d'une partie du personnel clé, des troubles sociaux, une inondation ou d'autres évènements qui perturbent le procédé de recherche ou modifient l'intervention. L'effet de ces évènements sur la fiabilité et la validité d'une étude est difficile ou impossible à mesurer. Au moment du choix d'un procédé de recherche, il est préférable de prendre certaines précautions sur le plan technique: 
a Recourir le plus possible à une répartition aléatoire des individus entre le groupe expérimental et le groupe temoin, à partir de la même population à étudier.

b S'il n'est pas possible d'effectuer une répartition aléatoire, le groupe témoin choisi devra posséder les mêmes caratéristiques sinon les plus proches de celles du groupe expérimental.

c Si les deux conditions précédentes ne sont pas réunies, il est recommandé d'utiliser des séries chronologiques, ce qui permet d'analyser la tendance avant et après l'introduction du programme.

d A défaut de série chronologique, essayer d'obtenir le maximum d'informations avant l'introduction du programme (pré-test) qui seront confrontées à celles du test final (approche utilisant un pré-test et un test final).

e S'il n'est pas possible d'effectuer un pré-test, le type d'analyse sera limité et il est recommandé de recourir à l'analyse multivariée.

f La question de l'exactitude des données doit être toujours présente à l'esprit. Les effets possibles des phénomènes perturbateurs doivent toujours être envisagés (tels que les effets dus à la conjoncture, à la selection, à la répétition, à la durée du projet ou liés aux modifications de l'outil de mesure). Ces effets peuvent expliquer certains résultats observés.

\section{$E$ Principes de base pour un "bon" procédé de recherche.}

1 Un bon procédé de recherche est celui qui répond aux critéres éthiques. Il ne doit pas aller à l'encontre des droits et de la dignité des personnes et il ne doit pas les priver d'un certain nombre de services ni de la qualité de ces services dont elles auraient bénéficié normalement.

2 Un bon procédé de recherche est celui qui permet d'obtenir les données les plus fiables possibles vu les contraintes financières, le temps imparti, le personnel et l'équipement disponibles.

3 Un bon procédé de recherche est celui qui permet de mesurer tout phénomène survenant sur le terrain, qu'il soit l'effet de l'intervention ou d' évènements imprévus et peutêtre perturbateurs.

4 Un bon procédé de recherche est celui qui permet au chercheur d'éviter de faire des déductions erronées en optant par exemple pour une hypothèse inexacte, ou en rejetant une hypothèse juste.

\section{F La règle des trois multiples}

Lorsqu'on choisit un procédé de recherche, il est toujours recommandé de penser à la règle des trois multiples lorsque les conditions le permettent.

1 Chercher à obtenir des sources multiples de données pour obtenir des informations sur la même variable.

2 Chercher à obtenir des mesures multiples dans la durée pour une même variable.

3 Chercher à obtenir la duplication multiple de l'intervention dans plusieurs sites différents.

L'utilisation de sources multiples de données répond à trois objectifs. Premièrement, l'exactitude d'une source peut être confrontée à l'autre. Deuxièmement, chaque source peut fournir des informations supplémentaires sur un phénomène ou mettre en valeur la relation existant entre plusieurs phénomènes. Troisièmement, le recours à des sources multiples fournit l'occasion d'obtenir des informations qualitatives sur le processus de recherche, ce qui peut servir à déterminer pourquoi et comment l'intervention a obtenu ou pas des résultats.

Des mesures multiples dans la durée de la même variable peut fournir des informations sur les tendances avant, pendant et après l'introduction de l'intervention. Ce type d'information peut 
être extrêmement utile pour les études sur le terrain. une variation soudaine des tendances peut être une indication que des facteurs extérieurs à l'étude affectent une zone ou un groupe expérimental.

La duplication multiple d'une intervention dans des sites différents peut aider à déterminer jusqu'à quel point les effets d'une intervention sont particuliers à une région et à un groupe de population, ou s'ils peuvent être généralisés à d'autres régions et groupes. Lorsqu'on a recours à la duplication multiple, l'idéal est d'effectuer dans plusieurs régions une ou plusieurs études de suivi ayant les mêmes objectifs et procédés de recherche. Dans la pratique, étant donné les contraintes financières et le temps limité, une telle duplication multiple est difficile à mettre en place. Une alternative consiste à introduire l'intervention dans différents sites en même temps. Une telle procédure non seulement aide à déterminer jusqu'à quel point l'impact d'une intervention peut être généralisé mais elle fournit aussi la garantie que si une zone expérimentale est perturbée par une inondation, des émeutes, des retards adminitratifs, des grèves ou autres, l'étude peut au moins se poursuivre dans d'autres zones.

\section{En résumé-procédés de recherche}

1 Le choix du procédé de recherche dépend dans une large mesure des objectifs de l'étude. L'examen de ces derniers doit aboutir aux interrogations suivantes:

a Veut-on évaluer un programme? Si oui, on aura recours à un procédé expérimental ou quasiexpérimental.

b Veut-on simplement décrire un phénomène particulier? Si c'est un évènement qui a déjà eu lieu, on peut utiliser un procédé avec uniquement un test final $\left(\mathrm{O}_{1}\right)$ et recourir à une analyse multivariée. Si c'est un évènement qui n'a pas encore eu lieu, on peut utiliser un procédé avec un test final et pré-test $\left(O_{1} \times O_{2}\right)$, ou recourir à une série chronologique $\left(O_{1} O_{2} O_{3} \mathrm{X}\right.$ $\mathrm{O}_{4} \mathrm{O}_{5} \mathrm{O}_{6}$ ) ou enfin à un groupe témoin pouvant avoir des caractéristiques différentes:

$$
-\frac{O_{1} \times O_{2}}{O_{3} O_{4}}--
$$

c Veut-on évaluer les effets d'un système particulier de prestation de services de planification familiale? Si oui, les données observées sur le groupe expérimental devront être confrontées à celles d'un groupe témoin choisi au hasard, sinon, à un groupe présentant un degré important de similitude au groupe expérimental.

2 Etablir ensuite, à l'aide des 0 et des $X$ le diagramme approprié de l'approche choisie. Ne pas oublier que les approches présentées ci-dessus peuvent se prêter à de multiples variations.

3 Prendre en considération, s'il y a lieu, les répercussions éthiques du procédé.

4 S'assurer que les ressources disponibles sont suffisantes pour la mise en place du procédé de recherche.

5 Examiner la liste des phénomènes perturbateurs possibles en distinguant ceux qui sont sous contrôle de ceux qui ne le sont pas. 


\section{Echantillonnage}

\section{A Domaines de recherche}

Les individus constituent les unités statistiques de base le plus souvent utilisées dans la recherche opérationnelle en matière de planification familiale. Pour étudier l'impact de l'introduction d'un nouveau contraceptif par exemple, on peut l'introduire dans un groupe donné, et ne pas le fournir à un autre. Plus tard, par interview, on peut obtenir des informations sur la pratique contraceptive des femmes de chacun des deux groupes. Si les deux groupes ont été formés au hasard, on peut vérifier l'hypothèse selon laquelle l'introduction de la nouvelle méthode tend à augmenter le niveau de la pratique contraceptrice. Dans ce type d'étude, la disponibilité et l'utilisation du nouveau contraceptif sont considérées comme des caractéristiques individuelles liées aux femmes interrogées. Chaque femme est considérée comme un sujet expérimental. Du moment qu'on interroge les femmes, on peut aussi les appeler "enquêtées." D'une manière plus générale on peut aussi les considérer comme des "cas" étant donné que les études ne sont pas toujours des expériences ou des enquêtes.

Les unités statistiques peuvent aussi être constituées par des ensembles ou des groupes d'individus. Ainsi la relation entre l'introduction du nouveau contraceptif et le niveau de la pratique contraceptive peut être étudiée à l'échelle communautaire. Si les cas à étudier sont constitués par des villages entiers (et non des individus), le nouveau contraceptif peut être considéré comme "disponible" s'il est offert dans une clinique se situant à une distance donnée du village; la pratique contraceptive pourrait être mesurée par les taux de prévalence à l'échelle du village (pourcentage des femmes mariées en âge de reproduction utilisant une méthode). Les unités peuvent aussi être constituées par des structures telles que les cliniques. Par exemple, une étude des cliniques pourrait être menée pour déterminer comment l'offre d'une nouvelle méthode contraceptive influence le choix de méthode par les nouvelles contraceptrices.

En général, l'unité statistique de base est déterminée par la nature du projet et par ses objectifs: ce sont les ménages cibles pour les projets ayant comme objectif l'amélioration de leurs connaissances et de leurs attitudes, ce sont les agents de terrain pour les projets ayant comme objectif la formation et le perfectionnement du personnel, et ce sont les cliniques pour les projets ayant pour objectif la comparaison de ses performances cliniques. Certaines études peuvent comporter différents types d'unités statistiques de base. Ainsi, une étude visant à vérifier les effets d'un nouveau programme de formation du personnel de terrain peut s'intérresser aussi bien aux agents de terrain qu'aux ménages désservis. Dans ce cas, la collecte, le traitement et l'analyse des données devront être effectués à deux niveaux, à savoir, celui du personnel et celui des ménages.

\section{B Méthodes de sondage}

Après le choix de l'unité statistique de base, l'étape suivante consiste à déterminer comment les unités vont être tirées. Il est utile de distinguer à cet effet, la population totale et l'échantillon qui sera effectivement observé. La population totale est constituée par des éléments qui sont tous des cas potentiels. Pour certaines études, les effectifs de la population sont si réduits qu'il est souhaitable 
et parfois obligatoire d'étudier l'ensemble de la population. Par exemple, si l'on veut étudier le système central de gestion d'un programme national dont il n'y a que 20 à 30 hauts responsables, 100 pourcent de l'échantillon devrait être tiré (l'ensemble des hauts responsables). De même, si le programme ne comporte que cinq équipes mobiles, l'étude concernant ces équipes doit couvrir l'ensemble des équipes mobiles. L'échantillon à 100 pourcent est parfois préférable même si la population à étudier est quelque peu nombreuse. Il en est ainsi, par exemple, si l'on veut étudier l'impact d'un programme donné sur la répartition par âge des acceptrices. On peut dans ce cas, étudier l'ensemble des fiches des acceptrices de la clinique surtout si le système de traitement est informatisé. De même, on peut s'intéresser à l'ensemble de la clientèle si l'on veut étudier les performances des cliniques.

Cependant, les études concernent souvent des effectifs tellement élevés de population, de régions ou de centres de prestation de services qu'il est nécéssaire de recourir à des échantillons inférieurs à 100 pourcent pour les représenter. Ainsi, pour effectuer une enquête sur la clientèle des cliniques du programme en 1982, la population qui nous intéresse est représentée par l'ensemble des personnes inscrites comme contraceptrices dans différentes cliniques pendant l'année 1982. Si les effectifs sont nombreux, 800,000 personnes par exemple, les interroger toutes serait hors de prix, interminable et inutile. La pratique courante consiste à interroger un échantillon relativement réduit des contraceptrices et de considérer les données observées comme représentatives de l'ensemble de la population. Si l'échantillon est vraiment représentatif et comprend plusieurs centaines de cas ou davantage, les résultats seront très semblables aux résultats qu'aurait donné la population entière.

Il existe deux types fondamentaux de sondages: (1) le sondage probabiliste, et (2) le sondage non probabiliste.

\section{Sondage probabiliste}

On préfère souvent le sondage probabiliste qui a plus de chances que le sondage non probabiliste de fournir un échantillon réellement représentatif de la population dont il est tiré. Le critère essentiel du sondage probabiliste est le fait d'attribuer à chaque élément de la population totale (c'est-àdire à chaque couple, à chaque agent, à chaque clinique, etc.) une probabilité donnée de figurer dans l'échantillon (si chaque élément possède une probabilité égale d'être tirée, l'échantillon est appelé auto-pondéré, et les données peuvent être analysées telles quelles et considérées comme représentatives). Il existe plusieurs types de sondages probabilistes dont les plus courants sont les suivants:

\section{a Sondage aléatoire simple}

Selon ce procédé, chaque élément de la population est affecté d'un numéro d'ordre unique. On tire ensuite les éléments, un à un, à l'aide d'une table de nombres au hasard ou bien de techniques de loterie, et ceci jusqu'à l'obtention de l'effectif desiré. Ce procédé devient fastidieux si l'échantillon est grand.

\section{b Sondage systématique}

Cette variante du sondage aléatoire simple est moins longue et plus facile à réaliser. En divisant l'effectif estimé de la population totale par la taille desirée de l'échantillon, on obtient le pas de sondage (appelons-le $n$ ). Pour tirer l'échantillon, on établit une liste de tous les éléments de la population et on tire tous les nièmes cas en commençant par un nombre au hasard compris entre 1 et $n$.

Alors que le sondage aléatoire simple exige que l'on ait à l'avance la liste de tous les éléments constitutifs de la population (appelée la base de sondage), le sondage systématique permet la réalisation simultanée de la base de sondage et de l'échantillon. Cela revient donc beaucoup moins cher. Il est préférable, naturellement, de disposer à l'avance de la base de sondage et d'effectuer un sondage systématique afin d'obtenir précisément la taille voulue de l'échantillon. Si l'effectif total de la population n'est pas connu à l'avance, la taille de l'échantillon ne peut pas l'être non plus. Supposons, par exemple, qu'on veuille tirer un échantillon de 20 ménages dans un village. On doit connaître le nombre total des ménages du village, sinon on doit en faire l'estimation. Si on l'estime à 200 ménages, le pas de sondage est 10; ce qui veut dire qu'au fur et à mesure qu'on établit la liste 
des ménages, on interroge un ménage sur 10. Si l'estimation est trop forte, l'échantillon sera d'une taille plus grande que prévue et si elle est trop faible, la taille de l'échantillon sera plus petite que prévue. Il est toujours préférable de prévoir une taille d'échantillon plus élevée que celle dont on a besoin. Il vaut mieux également prévoir le budget pour un échantillon plus grand que celui prévu.

\section{c Stratification}

On peut recourir à la stratification aussi bien avec le sondage aléatoire simple qu'avec le sondage systématique si on veut s'assurer de la représentativité de l'échantillon au niveau de certains sousensembles de la population étudiée. Par exemple, si on désire aboutir à une structure par âge de l'échantillon identique à celle de la population étudiée, on peut effectuer une répartition par groupes d'âge de cette population-stratification selon l'âge-et tirer un échantillon dans chaque strate ou groupe d'âge. Si la fraction de montage utilisée est la même pour toutes les strates, la structure par âge de l'échantillon sera identique à celle de la population étudiée. Il est souvent utile, toutefois, de faire appel à des fractions différentes de sondage pour chaque strate. Par exemple, pour une étude sur les personnes qui adoptent la planification familiale, il peut être souhaitable d'aboutir à un échantillon comprenant des effectifs identiques de couples utilisant la pilule, le condom, et la stérilisation, même si les effectifs réels sont différents. Pour celà, on effectue une stratification selon la méthode utilisée, et on tire un échantillon dans chaque strate à l'aide de fractions différentes de sondage.

\section{d Sondage par grappe}

On peut encore simplifier l'opération d'échantillonnage en tirant des grappes (d'éléments selon un sondage aléatoire simple, ou systématique ou avec stratification) et enquêter l'ensemble de la population de chaque grappe échantillon. La plupart du temps, les grappes sont constituées par des aires géographiques (provinces, districts, villages) ou des unités telles que des cliniques, des clubs ou des groupes d'agents de terrain. Ainsi, pour obtenir un échantillon de femmes mariées en âge de reproduction (FMAR) en milieu rural, on peut tirer d'abord un échantillon des groupes de villages (grappes) et enquêter ensuite l'ensemble des FMAR de ces grappes. Les inconvénients principaux de cette technique sont (1) les complications supplémentaires dans les calculs mathématiques de la variance de l'échantillon et par conséquent des tests de signification statistique des résultats; et (2) l'obligation de recourir à des effectifs echantillons plus élevés pour atteindre des degrés de signification statistique suffisants à tous les niveaux.

\section{e Sondage à plusieurs degrés}

La technique d'échantillonnage la plus couramment utilisée pour les populations nombreuses et diversifiées est le sondage à plusieurs degrés. Le premier degré de sondage consiste à former des grappes naturelles et d'en tirer un échantillon. Le second degré consiste à tirer un échantillon d'éléments (enquêtes, cas) au niveau de chaque grappe échantillon. Plus que deux degrés sont parfois nécessaires. Ce sont par exemple les provinces au permier degré, les districts dans les provinces au second degré, les villages dans les districts au troisième degré et enfin les personnes dans les villages au quatrième degré. Les différentes techniques de sondage peuvent être utilisées à chaque degré, à savoir, le sondage aléatoire simple, systématique, ou avec stratification.

\section{f Sondage avec probabilité proportionnelle à la taille (PPT)}

Ce procédé est une variante du sondage à plusieurs degrés. La méthode consiste à tirer chacune des grappes avec une probabilité proportionnelle à sa taille et de tirer un nombre égal d'éléments dans chaque grappe d'échantillon. La technique du sondage PPT est utile lorsque les grappes sont de tailles différentes; cela permet de réduire aussi bien la variance de l'échantillon que les coûts de la collecte des données.

\section{Sondage non probabiliste}

Le sondage non probabiliste (ou raisonné) consiste à obtenir un échantillon sans probabilité fixé d'avance. Un sondage non probabiliste peut être accidentel (on prend les cas que l'on trouve) ou délibéré (on choisit certains types de cas). Les procédés non probabilistes ne sont pas valables pour obtenir des échantillons réellement représentatifs. De tels procédés aboutissent presque toujours à 
une sur-représentation ou une sous-représentation de certains éléments de la population étudiée. Ainsi, faute de probabilité de tirage, il n'est pas possible de corriger les distorsions possibles de l'échantillon.

En dépit de ces inconvénients, le sondage non probabiliste constitue, dans certains cas, l'unique possibilité de collecte des données et notamment quand il s'avère difficile, voire très coûteux, de recourir à un sondage probabiliste. Soit le cas, par exemple, d'un ministère qui a intégré la planification familiale dans les activités courantes de ses agents de terrain et qui désire en faire une évaluation. Si le budget de recherche ne permet pas d'effectuer les déplacements nécessaires pour couvrir l'ensemble du pays, on peut choisir d'étudier uniquement les performances des agents dans les régions les plus proches, même si leur expérience est relativement différente de celles des agents dans les régions plus éloignées.

Il est parfois impossible ou trop coûteux d'observer à l'aide d'un sondage probabiliste l'effectif nécessaire de cas à analyser. Par exemple, si l'objectif de l'étude est d'analyser l'influence d'un programme de radio sur les perceptions et les attitudes des auditeurs en matière de planification familiale, il serait très coûteux de tirer un échantillon probabiliste des auditeurs. Il faudrait, en effet, établir une liste complète des auditeurs dans la région de diffusion (ou un échantillon représentant les sous-ensembles de la région), afin de tirer ensuite un échantillon représentatif des auditeurs. Si les fonds disponibles ne permettent pas de réaliser un sondage aussi coûteux, on peut faire du porte-à-porte et interroger au fur et à mesure les auditeurs ainsi rencontrés jusqu'à l'obtention de l'effectif désiré. Les résultats d'un tel échantillon devront être analysés avec prudence vu la relation probable entre la composition de l'échantillon et la facilité d'accès aux logements. Ainsi, les auditeurs des zones rurales ou d'habitat précaire peuvent être sous-répresentés car ils sont relativement plus difficiles d'accès que les habitants en milieu urbain ou appartemant à des milieux sociaux plus élevés.

Si le sondage non probabiliste s'impose, il faut minimiser les distortions possible de l'échantillon. Si on connait certaines caractéristiques de la population à étudier, il faudra s'efforcer d'inclure dans l'échantillon toutes les catégories de personnes selon leur proportion dans la population. Si on sait par exemple, que le tiers de la population vit en milieu urbain et deux tiers en milieu rural, on formera l'échantillon dans les mêmes proportions. Si on connait la structure par âge de la population et qu'on pense qu'elle est en relation avec le phénomène à étudier, on peut fixer d'avance les effectifs de l'échantillon dans chaque tranche d'âge. Ce procédé est appelé le sondage par quotas car on fixe d'avance le quota de chaque sous-ensemble dans l'échantillon final.

Si l'étude vise surtout à obtenir un éventail le plus large possible de données qualitatives chez un groupe de personnes (par exemple, l'analyse approfondie de l'application d'une stratégie donnée, la connaissance de tout l'éventail des problèmes à résoudre, ou la recherche de toutes les solutions possibles aux problèmes existants) le sondage non probabiliste peut s'avérer la meilleure méthode à utiliser. Cela permet au chercheur de choisir délibérément la gamme voulue des personnes qui répondront clairement et facilement à ses questions aussi complexes soient-elles. Ainsi, les ethnologues obtiennent les données relatives à la collectivité par le canal des personnes-clés choisies parmi les éléments informés de la population et appartenant aux divers sous-ensembles de la collectivité.

\section{Taille de I'échantillon}

La taille de l'échantillon doit être fixee en tenant compte de deux criteres: (1) les ressources disponibles dont dépend la taille maximum; et, (2) les besoins du plan d'analyse prévu dont dépend la taille minimum de l'échantillon. Si par exemple, le nombre d'enquêteurs formés pour mener une enquête pendant une période donnée est juste suffisant pour effectuer un certain nombre d'interviews, la taille de l'échantillon ne peut dépasser ce nombre. Par ailleurs, la taille de l'échantillon doit ètre suffisante (1) pour permettre une analyse correcte des tableaux croisés; (2) pour fournir le degré de précision voulu dans le calcul des proportions; et (3) pour rendre possible le calcul des tests de signification statistique des différences observées des proportions. Si les ressources disponibles pour l'étude ne permettent pas la réalisation d'un échantillon de taille suffisante, le chercheur devrait soit trouver des ressources supplémentaires, soit modifier son plan d'analyses des données. 
FIGURE 8.1

La pratique contraceptive selon le niveau d'instruction

\begin{tabular}{|c|c|c|c|c|}
\hline \multirow[b]{2}{*}{$\begin{array}{l}\text { Pratique } \\
\text { contraceptive }\end{array}$} & \multicolumn{4}{|c|}{ Niveau d'instruction } \\
\hline & $\begin{array}{c}\text { Enseignement } \\
\text { supérieur } \\
(5 \%) \\
\end{array}$ & $\begin{array}{c}\text { Enseignement } \\
\text { secondaire } \\
(15 \%)\end{array}$ & $\begin{array}{c}\text { Enseignement } \\
\text { primaire } \\
(40 \%)\end{array}$ & $\begin{array}{c}\text { Sans } \\
\text { instruction } \\
(40 \%) \\
\end{array}$ \\
\hline \multicolumn{5}{|l|}{$\begin{array}{l}\text { Pratiquant la contra- } \\
\text { ception }(50 \%)\end{array}$} \\
\hline \multicolumn{5}{|l|}{$\begin{array}{l}\text { Ayant pratiqué la } \\
\text { contraception }(20 \%)\end{array}$} \\
\hline $\begin{array}{l}\text { N'ayant jamais pra- } \\
\text { tiqué la contracep- } \\
\text { tion }(30 \%)\end{array}$ & & & & \\
\hline
\end{tabular}

Si on compte produire pour l'analyse des tableaux de variables croisées, la taille de l'échantillon devra être fixée compte tenu de deux critères. Tout d'abord, les fréquences de chaque variable indépendante dans un tableau croisé devront être supérieurs ou égales à 50, car les pourcentages calculés sur des effectifs inférieurs à 50 ne sont pas fiables. La taille minimum requise de l'échantillon pour observer au moins 50 cas dans chaque catégorie de la variable est calculée en divisant 50 par la proportion $\left(P_{\mathrm{S}}\right)$ mesurant la fréquence relative la plus faible de la variable.

$$
m=\frac{50}{P_{s}}
$$

Ensuite, le nombre d'observations prévues dans chacune des cases du tableau devra atteindre au moins un effectif égal à cinq. En conséquence, la taille de l'échantillon est calculée en divisant cinq par le produit des fréquences relatives les plus faibles des deux variable du tableau. On peut illustrer ces deux points par la figure 8.1 qui mesure la pratique contraceptive selon le niveau d'instruction.

Le niveau d'instruction constitue dans ce tableau la variable explicative (indépendante). Si on veut aussi au moins 50 observations pour la fréquence relative la plus faible de la variable niveau d'instruction (c'est-à-dire la catégorie: "enseignement supérieur" qui ne représente que 5 pourcent de l'ensemble) on divise 50 par 5 pourcent $(n=50 / 0,05=1000$ observations nécessaires). Pour le calcul de la taille minimum de l'échantillon, afin d'avoir au moins 5 observations dans chaque case, on divise 5 par le produit des fréquences relatives les plus faibles de chaque variable (soit ici 0,05 pour la catégorie "enseignement supérieur" et 0,20 pour la catégorie "ayant pratiqué la contraception").

$$
n=\frac{5}{(0,05)(20)}=500 \text { observations nécessaires }
$$

Comme la taille nécessaire de l'échantillon doit être fixée en tenant compte des deux critères ( 50 pour chaque catégorie de la variable et 5 pour chaque case), c'est la taille supérieure qui sera choisie, soit $n=1.000$ plutôt que $n=500$.

Pour calculer la taille minimum de l'échantillon dont vous avez besoin pour estimer les proportions avec précision, vous devez d'abord répondre aux questions suivantes:

1 Quelles sont les estimations les plus raisonnables des proportions-clés à mesurer par l'étude. Si vous voulez étudier la proportion d'utilisation de la contraception, vous devez estimer la proportion que vous allez obtenir. S'il n'est pas possible de deviner, le moyen le plus sur est de l'estimer à 0,5 (50 pourcent), ce qui maximise la variance et fournit un échantillon de taille suffisante.

2 Quel est le degré de précision voulu? Quels sont les écarts acceptables entre les proportions observées et les proportions réelles? Si le taux de prévalence dans l'échantillon est estimé 0,50 , avec quelle marge de confiance ce résultat sera-t-il accepté, à 1 pourcent ou à 5 pourcent (en général, une marge de 0,05 ou 0,01 )? Si on désire un degré de précision élevé (par exemple 0,01) l'échantillon devra être d'une taille beaucoup plus grande qu'avec un degré de précision relativement plus faible $(0,05$ par exemple). 
3 Quel est le degré de confiance désiré? Dans quelle mesure peut-on être certain des résultats obtenus? On désire, en général, un degré de confiance de 95 pourcent.

4 Quel est l'effectif de la population que l'échantillon est censé représenter? S'il est supérieur à 10.000, la limite exacte de l'échantillon a peu d'importance. Par contre s'il est inférieur à 10.000 , la taille de l'échantillon devrait être plus petite.

5 Si on cherche à mesurer des différences entre deux sous-ensembles par comparaison de leur proportion, quelle est la différence minimum qui sera considérée comme statistiquement significative? Par exemple, si l'on compare la proportion d'utilisation de la contraception dans un groupe expérimental avec celle d'un groupe témoin et qu'on trouve une différence de 5 pourcent, un écart aussi faible sera-t-il considéré comme statistiquement significatif? Plus l'écart considéré comme significatif est petit, plus la taille des sousensembles échantillons doit être grande.

La taille de l'échantillon, obtenue en fonction des réponses à ces cinq interrogations et permettant de fournir les proportions voulues avec la précision voulue et le degré de confiance désiré, sera calculée pour des populations supérieures à 10.000 par la formule:

$$
n=\frac{z^{2} p q}{d^{2}}
$$

Avec:

$n$ = la taille désirée de l'échantillon (quand l'effectif de la population est supérieur à 10.000).

$\mathrm{z}=$ L'écart, fixé en général à 1,96 (ou plus simplement à 2,0 ) qui correspond à un degré de confiance de 95 pourcent.

$p=$ Proportion de la population cible ayant une caractéristique donnée. S'il n'existe pas d'estimation disponible, on peut utiliser 50 pourcent $(0,50)$.

$q=1,0-p$

$d=$ degré de précision voulu, en général 0,05 et parfois 0,02

A titre d'exemple, si l'on estime la proportion de la population ayant une caractéristique donnée à 0,50 , la valeur de $z$ égale à 1,96 , et le degré de précision 0,05 , la taille de l'échantillon sera:

$$
\begin{aligned}
n & =\frac{(1,96)^{2}(0,50)(0,50)}{(0,05)^{2}} \\
& =384
\end{aligned}
$$

Si on prend pour $\mathrm{z}$ la valeur plus commode de 2, la taille sera:

$$
\begin{aligned}
n & =\frac{(2)^{2}(0,50)(0,50)}{(0,05)^{2}} \\
& =400
\end{aligned}
$$

On remarque ici que le numérateur est égal à 1,0; ce qui veut dire que si la proportion est estimée à 0,50 et que le degré de confiance est de 95 pourcent, on peut obtenir la taille de l'échantillon par la formule:

$$
n=\frac{1,0}{d^{2}}
$$

Si l'effectif $N$ de la population étudiée est inférieur à 10.000, la taille de l'échantillon sera plus réduite, et sera calculée comme suit:

$$
n_{f}=\frac{n}{1+\left(\frac{n}{N}\right)}
$$

Avec:

$$
\begin{aligned}
n_{f}= & \text { la taille désirée de l'échantillon (lorsque l'effectif de la population est inférieur à } \\
& 10.000 \text { ) }
\end{aligned}
$$


$n=$ la taille désirée de l'échantillon (lorsque l'effectif de la population est supérieur à $10.000)$

$N=$ l'effectif estimé de la population.

Par exemple, si la taille $n^{\prime}$ a été estimée à 400 et si l'effectif de la population étudiée est égal à 1.000, on peut calculer $n_{f}$ comme suit:

$$
\begin{aligned}
n_{f} & =\frac{400}{1+\left(\frac{400}{1.000}\right)} \\
& =\frac{400}{1,4} \\
& =286
\end{aligned}
$$

Si on veut mesurer la signification statistique de la différence (d') d'une porportion dans deux sous-échantillons en supposant un nombre égal d'observations $\left(n_{2}=n_{2}=n\right.$ ) dans chaque souséchantillon, la formule qui donne n'est identique à la précédente:

$$
\frac{n^{\prime}=2 z^{2} p q}{\left(d^{\prime}\right)^{2}}
$$

Si on veut par exemple, comparer la proposition $p$ d'utilisation de la contraception estimée à 0,40 dans un groupe expérimental à celle d'un groupe témoin, et qu'on décide qu'une différence observée de 0,10 ou plus est significative avec un niveau de 0,05, la taille de l'échantillon sera calculée comme suit:

$$
n^{\prime}=\frac{2(1,96)^{2}(0,40)(0,60)}{(0,10)^{2}}=184
$$

On a donc besoin d'un échantillon expérimental de taille égale à 184 et d'un échantillon témoin de taille égale à 184. On remarque, ici aussi, que la formule sera encore simplifiée si on fixe $p$ à 0,50 et $\mathrm{z}$ à 2,0 soit:

$$
n^{\prime}=\frac{2}{\left(d^{\prime}\right)^{2}}
$$

Ce qui donne, pour le même exemple:

$$
\begin{aligned}
n^{\prime} & =\frac{2}{(0,10)^{2}} \\
& =200
\end{aligned}
$$

Si on ne peut pas assurer l'hypothèse d'une même taille des deux groupes échantillons ou si l'effectif des populations dont sont tirés les deux échantillons est largement inférieur à 10.000 , l'estimation de la taille de l'échantillon est plus compliquée. Dans de tels cas, et chaque fois qu'il existe des difficultés particulières, il vaut mieux consulter un statisticien. 


\section{En résumé-échantillonnage}

1 Décider d'abord de l'opportunité de tirage d'un échantillon et dans l'affirmative, opter pour un sondage probabiliste ou un sondage non probabiliste. Cette decision doit être prise compte tenu des objectifs de l'étude, du degré de représentativité des données et des moyens disponibles relatifs aux coûts, du temps et du personnel.

2 Calculer la taille de l'échantillon. On peut utiliser les formules présentées ci-dessus, en recourant aux services d'un statisticien si possible. Ce dernier aura besoin d'une estimation des proportions, du degré de précision desiré, du niveau de confiance utilisé, et de l'effectif approximatif de la population dont sera tiré l'échantillon.

3 Les principes suivants doivent être respectés:

a Une taille d'échantillon relativement plus grande fournira des résultais plus précis mais coûtera plus cher qu'un échantillon de taille plus réduite.

b Les données quantitatives fournies par un sondage probabiliste seront plus représentatives que celles fournies par un sondage non-probabiliste; on peut recourir à ce dernier pour la collecte de données qualitatives à partir d'échantillons de tailles réduites.

c Dans le cas ou certains sous-groupes de l'échantillon devront être particulièrement analysés, la taille de l'échantillon devra être augmenté en conséquence. Par exemple, si on veut étudier les caractéristiques des utilisateurs de contraception, la taille de l'échantillon peut être de 400; mais si la même analyse doit être menée selon la méthode utilisée, on doit augmenter la taille de l'échantillon afin de garantir une représentativité suffisante au niveau des sous-groupes échantillons. Même si on estime que la signification statistique n'a pas beaucoup d'importance, le plus petit des sous-groupes à étudier doit comporter au moins 50 observations si on veut obtenir des pourcentages quelque peu crédibles. 


\section{Collecte des données}

La collecte des données peut se faire de plusieurs manières. La méthode choisie dépend des objectifs, de la méthodologie de l'étude et des disponibilités en matière de personnel, de temps et d'argent. Le choix de la meilleure méthode dépend, entre autres, du type de données à recueillir, à savoir, les données quantitatives avec un certain degré de précision ou les données qualitatives fournissant des informations de type descriptif. La plupart des recherches opérationnelles cherchent à mesurer quantitativement le fonctionnement du programme, mais certaines cherchent aussi (ou devraient le faire) à obtenir des informations de type qualitatif soit sur les processus (comment, par exemple un projet est concrètement mis en application sur le terrain, ou la manière dont les couples décident d'adopter une méthode contraceptive de préférence aux autres méthodes disponibles), soit sur les perceptions (par exemple, le mode d'emploi des méthodes chez la clientèle potentielle ainsi que de leurs effets possibles, ou les problèmes que la clientèle associe avec le programme en cours). Souvent, les objectifs de l'étude exigent la collecte aussi bien des données quantitatives que qualitatives, et il faudrait recourir alors à plus d'une méthode de collecte des données.

\section{A Données quantitatives}

La méthode la plus couramment utilisée pour la collecte des données quantitatives est l'interview structurée. D'autres moyens peuvent toutefois être employés, telles que l'interview indirecte, les statistiques ou certaines sources secondaires telles que les données des recensements d'état civil, de certains documents et de rapports divers. Si les unités d'observation sont constituées par des ensembles géographiques ou par certaines structures (comme les villages, les districts, les cliniques ou les hôpitaux) plutôt que par des individus, les données quantitatives peuvent être constituées par les statistiques ou d'autres sources secondaires. A défaut de tels moyens, ces données pourraient être obtenues par l'interview de certains membres bien renseignés appartenant à ces unités.

\section{Interviews structurées}

On appelle enquêtes les opérations de collecte de données à partir d'interviews auprès de la population. Ces opérations sont appelées enquêtes par sondage si la collecte se fait sur un échantillon représentatif d'une population plus large. Si la taille de l'échantillon est suffisamment élevée pour des analyses statistiques, on a recours en général à des interviews structurées plutôt qu'à des interviews non structurées. En effet, le premier type est particulièrement adapté aux analyses quantitatives, alors que le second peut poser de sérieux problèmes de traitement et en particulier si l'échantillon est assez grand. Pour effectuer les interviews structurées, on utilise un questionnaire type (ou fiche d'interview) afin de poser les mêmes questions dans le même ordre. La formulation exacte des questions est indiquée à l'avance et l'enquêteur n'aura qu'à lire les questions à chaque personne interrogée. La préparation d'un questionnaire-type destiné à être utilisé par des enquêteurs doit obéir à certains principes:

a Utilisation d'un language simple, facilement compréhensible par les enquêtés.

b Utilisation des questions pré-codées chaque fois que possible afin de faciliter le transfert 
des données sur ordinateur et leur mise en tableau. Celà demande un plus grand effort pour la préparation de la fiche d'interview mais permet par contre une économie de temps et de travail pour le traitement et l'analyse des données.

c Eviter les questions délicates et embarrassantes. S'il s'avère nécessaire d'en poser une de ce genre, il faut la formuler avec autant de tact que possible et éviter de la placer au début du questionnaire avant que la personne ne soit complètement à l'aise.

d Ne pas demander plusieurs informations dans une même question. Ne pas demander, par exemple, "Est-ce que vous désirez avoir, vous et votre mari, un autre enfant?" La réponse "oui" ou "non" à cette question ne sera pas facilement interprétable si la femme interrogée et son mari ne sont pas d'accord sur le fait d'avoir un autre enfant. La réponse "oui" peut vouloir dire, "Je désire avoir un autre enfant" ou "mon mari désire avoir un autre enfant," ou encore, "mon mari et moi désirons avoir un autre enfant." Il vaudrait mieux dans ce cas, poser deux questions séparées: "Désirez-vous avoir un autre enfant?" et "Votre mari désire-t-il avoir un autre enfant?"

e Faire attention aux questions ambiguës. Si l'expression planification familiale, par exemple, à été déjà définie, on peut facilement comprendre le sens de la question "Quelle est la (ou les) méthode(s) de planification familiale que vous utilisez?" Mais si ce sont des femmes que l'on interroge, elles peuvent ne pas citer les méthodes masculines comme la vasectomie ou les condoms. Dans ce cas, la question devrait être "Quelle est la (les) méthode(s) de planification familiale que vous utilisez, vous ou votre mari?"

f Ne pas surcharger la fiche de l'interview par des questions sans beaucoup d'importance pour l'étude. La fiche doit être la plus courte possible afin d'éviter que la personne enquêtée ne s'ennuie et afin de simplifier le traitement et l'anályse des données.

g Vérifier qu'il y a bien toutes les questions relatives aux variables à étudier. S'assurer aussi que les données nécessaires pour le test de l'hypothèse peuvent être obtenues par les supports de collecte. Il est toujours utile de préparer une liste des variables clés de l'étude en indiquant où les données relatives à chacune d'entre elles pourront être obtenues.

h Commencer par les questions les plus faciles et ne passer aux plus difficiles ou plus délicates qu'une fois l'atmosphère de tension, ou même de suspicion, qui caractérise en général le début de toute interview, soit dépassée. Les premières minutes de l'interview doivent être consacrées à la prise de contact et la mise en confiance de la personne à interroger, ce qui peut être facilité par des premières questions simples et anodines.

i Chaque question doit être posée de la même façon à chaque personne interrogée. Si les mêmes interviews doivent être effectuées dans plusieurs langues, une version écrite dans chacune des langues principales devra être préparée et les enquêteurs devront s'y conformer mot-à-mot. Les traductions libres ne doivent pas être permises sauf quand les effectifs à interroger sont peu élevés et ne justifient pas les coûts de la traduction. On peut vérifier la qualité de chacune des versions en procédant à la traduction en sens inverse par des personnes qui ignorent la version originale. On peut ainsi vérifier si les questions ont gardé tout leur sens.

j Effectuer un pré-test du questionnaire dans des conditions réelles de terrain. L'opération du pré-test obéit à certaines règles:

1 Pour le pré-test, il n'est pas nécessaire d'interroger un grand nombre de personnes; 30 à 50 personnes sont en général suffisantes si elles sont choisies de manière telle qu'on y retrouve la diversité attendue de l'échantillon à étudier. C'est ainsi qu'il faudra interroger des jeunes et des plus agés, des urbains et des ruraux, des moins instruits aux plus instruits, des utilisateurs de la contraception et des non utilisateurs, etc.

2 Envisager la possibilité de plus d'un pré-test. Si le pré-test donne lieu à un nombre important de modifications dans le questionnaire, il vaut mieux effectuer un autre pré-test pour juger des résultats.

3 Le pré-test doit être terminé avant la formation des enquêteurs. On utilise pour cette opération les superviseurs de terrain qui seront ainsi plus au courant des objectifs de l'étude et plus à même d'aider à la formation des enquêteurs.

4 L'objectif principal du pré-test est de vérifier le degré de compréhension des questions par les personnes interrogées ainsi que de leur capacité de bien y répondre. 
Ainsi, le pré-test ne doit pas constituer un simple interrogatoire mais doit être suivi par une discussion sur certains aspects mal compris et certaines difficultés décelées au cours de l'interview.

k Assurer une formation complète à tous les enquêteurs. L'objectif de la formation est de familiariser les enquêteurs avec le contenu et l'intérêt des différentes questions grâce à des expériences pratiques et encadrées sur le terrain.

I Si une personne à interroger n'est pas disponible lors de la première visite effectuée par l'enquêteur, un rendez-vous pour une autre visite doit être pris. Deux rendez-vous au moins sont nécessaires avant d'abandonner définitivement l'interrogatoire de la personne échantillon.

m Si l'échantillon est limité, il peut s'avérer nécessaire de remplacer les cas non retrouvés. Dans ce cas, l'enquêteur doit procéder au remplacement selon des instructions précises que ce soit un tirage aléatoire ou par le choix de remplaçants ayant les mêmes caractéristiques que les personnes non retrouvées de l'échantillon. Toutefois, si l'échantillon est assez grand et qu'on peut tolérer une certaine déperdition, il vaut mieux s'abstenir d'effectuer les remplacements.

n Tout doit être tenté pour que l'interview s'effectue seul à seul avec la personne interrogée. La présence d'autres personnes peut avoir une influence sur la qualité des réponses. Une femme dont le mari ne sait pas qu'elle utilise des contraceptifs oraux, par exemple, risque de fournir une réponse toute autre selon que le mari soit présent ou non à l'interview.

o Vérifier toutes les fiches d'interview remplies aussitôt que possible pour détecter les erreurs, les omissions et les contradictions possibles. Une autre visite pourrait s'avérer nécessaire si certaines erreurs ne sont pas corrigées à temps. L'enquêteur devrait vérifier sur place le questionnaire, dès la fin de l'interview. La vérification du questionnaire et sa correction doit être refaite ensuite par le superviseur. Cette opération est appelée vérification de terrain.

\section{Statistiques de services}

Les organismes nationaux chargés de la planification familiale produisent de nombreuses statistiques de services. Certains ont mis en place un Système d'Observation et de Surveillance (SOS). La qualité de ces statistiques de services varie aussi bien d'un pays à l'autre qu'au niveau du pays même. Il faut donc les utiliser avec prudence. Ces statistiques peuvent souvent aider le chercheur à fixer les paramètres de son étude. Elles constituent aussi dans certains cas, une source de comparaison à l'échelle nationale de certains résultats obtenus par une recherche ponctuelle. Il est possible aussi dans le cadre de certains projets de recherche opérationelle, de mettre en circuit des fiches spéciales pour la collecte de certaines données qui ne sont pas disponibles par ailleurs.

\section{Interviews indirectes}

L'enquête par interview directe est toujours préférable au procédé de l'interview indirecte, si les moyens en termes de budget, de personnel et de temps sont disponibles. Vu le niveau d'instruction généralement bas, il est difficile, voire impossible, dans les pays en développement d'éffectuer des enquêtes au moyen de questionnaires remplis par les enquêteurs eux-mêmes. De plus, même lorsqu'il s'agit de personnes instruites, d'autres difficultés telles que l'incompréhension de certaines questions ou de certaines instructions, subsistent en l'absence des explications d'un enquêteur. Certaines parties du questionnaire risquent de ne pas être remplies et certains types d'instructions sont difficiles à incorporer ou peuvent créer des confusions (exemple: "Si la réponse à la question 12 est "oui" passer à la question 13, sinon passer à la question 18 ").

On peut recourir aux questionnaires remplis par les enquêtés eux-mêmes si ceux-ci, sachant lire et écrire, sont réunis ensemble dans une salle de classe ou un bureau par exemple, où ils peuvent écrire facilement. Ce type d'interviews peut être particulièrement indiqué dans l'évaluation des programmes d'éducation en matière de population ou des stages de formation.

Parfois les questionnaires sont envoyés par la poste aux personnes interrogées qui doivent les renvoyer une fois remplis. Ce moyen a l'avantage de ne pas coûter cher mais il a aussi tous les inconvénients cités ci-cessus avec en plus des risques élevés de non réponses. Généralement, les 
réponses reçues par poste n'excèdent pas la moitié des questionnaires envoyés, même auprès des lettres de rappel, ce qui nuit à la représentativité de l'échantillon et à la fiabilité des données quantitatives et risquent d'être d'utilisation limitée.

\section{Sources de collecte secondaires}

Outre les données recueillies spécialement pour l'étude, l'analyse gagne à inclure aussi les données fournies par les recensements récents, l'état civil, les statistiques officielles ainsi que les enquêtes précédentes. Une grande quantité de données relatives à la santé et à la planification familiale a été recueillie par des enquêtes internationales telles que l'Enquête Mondiale sur la Fécondité, L'Enquête sur la Prévalence Contraceptive, et la plus récente, l'Enquête Santé et Population.

\section{B Données qualitatives}

Les études descriptives utilisant des données de type qualificatif font appel à des techniques de collecte différent sensiblement de celles utilisées dans les études quantitatives. La majorité des études de recherche opérationnelle devrait avoir recours à une combinaison des méthodes qualitatives et quantitatives de collecte des données afin d'obtenir l'image la plus précise et réaliste de la situation d'un programme. Les méthodes quantitatives discutées plus haut sont importantes pour effectuer des prévisions, des projections et pour aboutir à des généralisations. Les méthodes qualitatives telles que les interviews non structurées, les interviews de groupes, l'observation directe, et l'analyse de contenu de documents sont importantes pour obtenir des données sur les processus du programme, à savoir comment et pourquoi un programme fonctionne d'une certaine mạnière, ainsi que sur les résultats inattendus.

\section{Interviews non structurées}

L'inconvénient majeur des données obtenues par la méthode de l'interview non structurée réside dans la qualité parfois superficielle des réponses. Pour pallier à ce défaut et obtenir des informations plus approfondies, on peut utiliser des questions ouvertes ou semi-fermées. On aboutit ainsi à ce qu'on appelle des interviews approfondies. Au lieu de poser des questions prises directement d'un questionnaire, l'enquêteur procède à un interrogatoire en partant d'un canevas de thèmes et de problèmes généraux à étudier. Les aspects qui n'apparaissent pas au debut de ces interviews sont simplement provoqués par un enchaînement de questions exploratrices.

La méthode de l'interview non structurée présente néanmoins les inconvénients suivants: (1) quantification délicate des réponses, celles-ci n'étant pas standardisées à l'avance; (2) niveau élevé de compétence et de pratique requis de la part des enquêteurs; (3) longs délais nécessités par l'analyse. De telles interviews portent en général sur des échantillons de faibles effectifs ( 20 à 30 personnes), vu le manque d'enquêteurs et d'analystes qualifiés ainsi que les coûts élevés de collecte et de traitement de telles enquêtes. Ce type d'interviews est souvent utilisé durant la phase exploratoire des études quantitatives afin de mettre au point les concepts et les hypothèses de recherche qui servent à établir les questionnaires. On se sert aussi de tels types d'interviews afin d'obtenir des données explicatives supplémentaires complétant les données relativement superficielles déjà fournies par une enquête à plus grande échelle.

Par exemple, on ne connaît que peu de chose au sujet des hommes qui demandent une vasectomie. Une interview non structurée pourrait contribuer à une meilleure connaissance des facteurs qui déterminent le choix de la vasectomie.

\section{Interviews de groupe (Focus Group Discussion)}

Une variante de la méthode de l'interview approfondie est de rassembler les personnes à interroger au sein de groupes de discussion (focus groups). Ceci permet d'obtenir des données aussi détaillées à moindre frais de personnel et de temps pour la collecte et l'analyse. L'enquêteur (ou l'animateur du groupe) fait appel aux mêmes techniques que celles de l'interview non structurée en partant d'un canevas de thèmes et de problèmes généraux à étudier et en suscitant des réponses plus détaillées grâce à une suite de questions. Les participants sont en général choisis de manière à 
refléter la diversité exigée par l'étude à mener. Par exemple, les participants à un groupe de discussion (focus group) pour une étude sur les méthodes de contraception doit inclure des échantillons bien choisis d'époux, d'épouses, d'utilisateurs actuels de chacune des méthodes contraceptrices, d'anciens utilisateurs ayant rompu l'utilisation de telles méthodes et des personnes qui n'en ont jamais utilisé.

\section{Observation directe}

L'observation directe est une technique qui permet d'obtenir aussi bien des données quantitatives que qualitatives et qui exige des périodes d'observation assez longues et du personnel et des analystes relativement compétents, ce qui entraine des coûts assez élevés par unité d'observation. Ainsi, cette méthode n'est utilisée que pour des études exploratoires à petite échelle. Elle se prête particulièrement bien à l'étude des réactions d'une communauté, par exemple, lors de l'introduction d'un nouveau programme. C'est la méthode typique des ethnologues dont la specialité est l'étude des communautés. Cette technique est particulièrement adaptée, par ailleurs, à l'étude du fonctionnement de certaines cliniques, de certaines activités d'agents de terrains ou bien de procédés administratifs en vigueur.

\section{Analyses de contenu de documents}

Dans les recherches opérationnelles en matière de planification familiale, l'analyse de contenu de certains documents n'est pas utilisée en tant que méthode isolée mais en tant que complément utile à d'autres types de collecte et d'analyse des données. On peut analyser, par exemple, le contenu de certains manuels d'enseignement utilisés au cours d'un stage de formation afin de voir le type de connaissance et d'aptitude que la formation est censée développer. Le chercheur peut aussi mettre au point les tests d'évaluation nécessaires pour le personnel ainsi formé. On peut aussi analyser le contenu des matériaux d'éducation, information et communication utilisés pour évaluer le degré d'adaptation des messages aux besoins du programme. Le contenu des divers rapports de recherche peut être aussi analysé afin de situer l'état actuel des connaissances sur un aspect donné et orienter ainsi les recherches futures. Les articles de presse ou des déclarations publiques faites par des personnalités politiques peuvent être examinés afin d'évaluer les attitudes en ce qui concerne la planification familiale.

\section{En résumé-la collecte des données}

1 Examen des objectifs, des hypothèses de recherches, ainsi que de la liste des variables dépendantes et indépendantes de l'étude. De quel type d'information a-t-on besoin? Quelles sont les techniques appropriées et possibles pour la collecte des données nécessaires? Certaines de ces données sont-elles déjà disponibles par ailleurs?

2 Si on pense réaliser la collecte des données par une enquête, les différents points figurant sous le titre "Interview structurée" doivent être examinés. Tenir compte des différentes phases nécessaires telles que la traduction, le pré-test, la formation des enquêteurs et donner des précisions quant aux visites de rappel et aux méthodes de remplacement.

3 Décrire les instruments de collecte potentiels (exemple: Fiche d'interview, manuel d'explication, manuel d'enquête) avec des exemples de questions à poser et particulièrement celles qui concernent les variables-clés dont dépendent les hypothèses de recherche. Indiquer les méthodes adoptées pour mesurer toutes les variables à étudier (en fournir au moins une description). Il serait utile aussi d'énumérer toutes les variables et de fournir pour chacune d'elles les questions qui leur sont relatives. Par exemple:

Variable 1: Pratique de la planification familiale

Q1: Avez-vous déjà utilisé une méthode de planification familiale?

Q2: Utilisez-vous actuellement l'une des méthodes de planification familiale?

Variable 2: Instruction

Q1: Jusqu'à quelle classe avez-vous étudié à l'école?

4 Décrire les techniques de collecte des données qui seront adoptées et joindre l'ensemble à la proposition de recherche. 


\section{Contrôle de la qualité des données}

Il existe différentes manières de contrôler la qualité des données d'une enquête.

1 Le chercheur a recours parfois à deux ou plusieurs questions relatives à une même donnée, la première au début de l'interview, par exemple, et la seconde à la fin. L'analyse des deux questions donnera une idée sur le degré de crédibilité des réponses. C'est là une méthode de vérifier la fiabilité des données.

2 Pour certaines questions difficiles ou délicates, l'enquêteur reçoit certaines instructions pour s'assurer que la réponse obtenue est correcte. Ainsi, il pose la même question sous une forme légèrement différente ou bien, il relit la réponse à la personne interrogée et lui demande si c'est bien exact. Si une femme, par exemple, déclare qu'elle a deux fils et trois filles, l'enquêteur peut lui dire "vous avez au total cinq enfants dont deux de sexe masculin et trois du sexe féminin, est-ce exact? Avez-vous d'autres enfants que vous avez oublié de mentionner?"

3 Des superviseurs de terrain doivent être employés pour aider les enquêteurs dans les situations délicates et vérifier qu'ils font bien leur travail. Parfois même les enquêteurs remplissent eux-mêmes les questionnaires dans les cafés! Le quota utilisé dans certaines études est d'un superviseur pour 5 enquêteurs.

4 Dans la plupart des enquêtes par interview, on recommence la même enquête auprès d'un certain pourcentage des mêmes personnes qui varie en général de 5 pourcent à 10 pourcent selon la taille de l'échantillon. Les données du premier passage sont ensuite confrontées à celles du deuxième passage pour vérifier le degré de crédibilité des réponses, ce qui constitue une autre manière de tester la fiabilité des données. Si on trouve des contradictions avec des questions aussi importantes que l'âge, l'état matrimonial ou la parité, c'est qu'il y a un problème avec le questionnaire, les enquêteurs, les méthodes de tabulation ou avec tout autre aspect de l'enquête.

5 Une fois terminé les opérations de collecte et de mise en tableaux des données, certains contrôles statistiques sont possibles pour détecter les erreurs et tester la crédibilité des réponses. L'analyse des fréquences de la variable paritée peut montrer que certaines femmes auraient déclaré avoir 18 ou 19 enfants vivants. Comme c'est très improbable, le chercheur peut, soit éliminer ces questionnaires, soit ne pas tenir compte des réponses à la question sur la parité, soit enfin refaire l'interview auprès de ces femmes ayant déclaré avoir 18 ou 19 enfants vivants.

\section{En résumé-contrôle de la qualité des données}

1 Décrire les méthodes à utiliser pour le contrôle de la qualité des données. Tenir compte des aspects suivants:

a Tester la crédibilité des réponses en posant plusieurs fois la même question dans le questionnaire.

b Recourir à des superviseurs pour encadrer les enquêteurs sur le terrain.

c Tester la crédibilité des réponses en effectuant une seconde interview auprès d'un pourcentage des personnes interrogées.

d Effectuer un recodage de certains questionnaires pour vérifier qu'il n'existe pas d'erreurs de codage.

e Analyser les fréquences de toutes les variables pour vérifier qu'il n'existe pas des codes inattendus ou des données aberrantes. 


\section{Caractère confidentiel des informations}

Il faut toujours respecter le caractère confidentiel des données fournies par les personnes interrógées. Certaines informations délicates ne doivent être demandées que lorsque c'est vraiment nécessaire. D'autre part, il vaut mieux recourir plutôt à des codes qu'à des noms. Assurer toute personne interrogée du caractère confidentiel des données. Ne laisser personne utiliser des données si cela peut nuire aux personnes interrogées. Le chercheur doit respecter le caractère confidentiel des informations obtenues. De même, si une personne refuse de répondre à l'interview, il faut respecter sa décision. Il est recommandé d'utiliser un formulaire spécial d'information et de libre consentement décrivant les objectifs de l'étude et d'obtenir l'accord des personnes enquêtées avant de les interroger.

\section{En résumé-caractère confidentiel des informations}

1 Décrire en détail la méthode adoptée pour garder le caractère confidentiel des informations.

2 Joindre au projet de recherche un exemplaire du formulaire d'information et de libre consentement. 


\section{Mise en tableaux et analyse des données}

Si l'étude n'avait pour objectif que la présentation de certains aspects de type qualitatif ou descriptif, l'analyse consisterait simplement à rédiger un rapport incorporant les données brutes arrangées selon un certain plan. Mais en général, la phase d'analyse est plus complexe, notamment dans les recherches opérationnelles qui exigent une certaine manipulation des données. Il s'agit, en premier lieu, de décider si les tableaux seront obtenus manuellement ou d'une manière automatique; de voir, en second lieu, comment les données doivent être transformées en vue de leur traitement; de choisir, en troisième lieu, les procédés statistiques à utiliser pour l'analyse des données; et enfin de présenter les principaux résultats dans un ou plusieurs rapports.

\section{A Tabulation: codage et vérification des données}

De nos jours et dans tous les pays, on trouve des micro-ordinateurs dans les universités, les instituts de recherche, les ministères de la santé et les organismes de planification familiale. Ces micro-ordinateurs sont devenus des outils essentiels aussi bien pour la tabulation que pour l'analyse des données obtenues à partir des recherches opérationnelles. Le traitement informatique est préférable même pour des études limitées qui utilisent des procédés statistiques relativement simples. Les micro-ordinateurs, portables ou pas, peuvent tirer des tableaux et effectuer des opérations statistiques beaucoup plus rapidement (et la plupart du temps avec beaucoup moins d'erreurs) qu'il n'est possible par voie manuelle.

Une fois qu'on a défini les types de tableaux à produire, on doit choisir les méthodes à utiliser pour la transformation de l'information brute, la mise en tableaux et l'analyse des tableaux. Que le traitement soit manuel ou automatique, les aspects non chiffrés qui devront être quantifiés pour l'analyse doivent être au préalable convertis en codes chiffrés. En outre, les données devront passer de l'état brut (symboles utilisés dans les questionnaires, transcriptions d'interviews, publications relatives aux recensements, etc.) à la forme adéquate pour leur mise en tableaux. Si on a recours à la méthode manuelle, il est souvent utile de transférer les données codés sur des cartes conçues à cet effet. Pour le traitement informatique, les données devront être transférées sur des fiches de codification puis sur bandes magnétiques, disques durs ou disquettes.

Les instructions pour le codage des données doivent être préparées; celà permettra de convertir chaque catégorie de réponse en un code chiffré unique. Si le nombre total des réponses possibles, pour une variable donnée, est inférieur à 10 (y compris les réponses de type "ne sais pas", "ne convient pas", "réponse inconnue", etc.) le code sera à un seul chiffre. Si le nombre total de réponses possibles est compris entre 10 et 99, le code sera composé de deux chiffres. Le code peut être composé de trois ou quatre chiffres ou même plus selon les besoins.

Une fois codées, les données doivent être ponctuellement vérifiées. Une partie du codage doit être refaite par un autre codeur afin de déceler les erreurs éventuelles en comparant les deux séries. En cas d'erreurs, le superviseur du codage se chargera d'effectuer les corrections nécessaires. Ces erreurs proviennent en général de mauvaises instructions pour le codage ou d'une formation insuffisante des codeurs.

Lorsque toutes les données ont été codées, elles doivent être intégralement vérifiées du point 
de vue du codage (même si les informations brutes ont déjà été verifiées sur le terrain). Le premier type d'erreurs à déceler concerne les aspects suivants:

1 Codes erronés-les codes qui ne correspondent pas aux codes indiqués dans les instructions de codage.

2 Omissions-par exemple, un enquêteur qui n'a pas respecté les instructions relatives aux SAUTS dans le questionnaire.

3 Incohérences-par exemple, l'âge au moment de l'enquête inférieur à l'âge au moment du mariage de la femme.

4 Invraisemblances-par exemple, une femme âgée de 25 ans et ayant 10 enfants vivants.

Une fois les erreurs trouvées, elles doivent être confrontées aux données d'origine et corrigées en conséquence. La liste des erreurs est plus facilement obtenue si la vérification est effectuée au moyen d'un ordinateur; une telle opération est appelée vérification par ordinateur par opposition à l'opération de vérification sur le terrain. En général, les données destinées à être traitées par voie informatique sont conservées sur bandes magnétiques, disques dures ou diskettes.

\section{En résumé-codage et vérification}

1 Décider si les données seront traitées manuellement ou par voie informatique. Prendre en considération:

a Les possibilités existantes

b Le type de données (quantitatives ou qualitatives)

c Type d'analyse à effectuer

d Personnel disponible

e Coûts

S'il est décidé de recourir à un ordinateur, vérifier si certains programmes informatiques (logiciels) et une assistance en matière de programmation existent.

2 Décider du type de données à coder. Certaines informations peuvent être laissées parfois telles quelles. Certaines questions ouvertes, même dans le cas d'enquêtes structurées, peuvent servir à illustrer l'analyse. Dans ce cas, préciser les aspects qui seront traités dans cette optique.

3. Indiquer dans la proposition de recherche quelles seront les mesures prises pour la vérification et la correction des données codées.

4 Indiquer les méthodes choisies pour la vérification ponctuelle ou intégrale du codage.

\section{B Plan d'analyse des données}

\section{Caractéristiques des données}

Le plan d'analyse constitue l'un des aspects les plus importants du protocole de recherche. L'analyse des données doit fournir des réponses à toutes les questions soulevées par l'étude. L'analyse fractionne les données. Celles-ci sont ordonnées, regroupées et classées. En général, le chercheur essaiera de dégager, à partir des données, les caractéristiques suivantes:

\section{a Caractéristiques de tendance centrale}

Le chercheur a comme objectif, par exemple, de connaître la parité moyenne d'un ensemble de femmes, la méthode contraceptive la plus fréquemment utilisée (le mode), ou le revenu médiane. La moyenne, le mode et la médiane constituent des caractéristiques de tendance centrale. La moyenne est simplement la moyenne arithmétique obtenue en divisant la somme des scores ou des caractéristiques individuelles par l'effectif total de la population étudiée. Le mode est constitué par la caractéristique la plus fréquemment observée. Quant à la médiane, elle est constituée par la 
valeur qui divise la distribution en deux parties égales. Ces indicateurs constituent différents moyens d'approche, légèrement différents, pour mesurer les caractéristiques de tendance centrale d'une population étudiée.

\section{b La variance des données}

Souvent, le chercheur pousse son analyse au-delà de la tendance moyenne et cherche à connaître la variance des données observées, c'est-à-dire la tendance des individus observés à s'écarter de la moyenne ou de la tendance centrale. $\mathrm{Si}$, par exemple, les acceptrices de pilules sont âgées en moyenne de 28 ans, on cherchera à connaître l'éventail selon l'âge, l'âge de la plus jeune acceptrice et celui de l'acceptrice la plus âgée. L'indicateur le plus utilisé à cet effet est constitué par l'écarttype. C'est l'écart moyen par rapport à la moyenne.

\section{c Différence entre les résultats}

La plupart du temps, on cherchera à connaître si les résultats observés ne sont pas simplement dus au hasard. Si on trouve, par exemple, un taux d'utilisation de la contraception de 43 pour cent chez un groupe expérimental de 1.000 femmes mariées, à comparer avec un taux de 21 pour cent chez un groupe témoin, dans quelle mesure ces taux sont-ils significativement différents et quelle est la part du hasard dans les différences observées? L'un des tests statistiques le plus fréquemment utilisé est celui du test $\mathbf{X}^{2}$ et du test $t$ qui mesure la différence entre les moyennes. De tels tests sont présentés, avec plus de détails, plus loin.

\section{d Relation entre les données}

Dans la plupart des études, on cherche à établir les relations, si elles existent, entre les différentes variables. Est-ce que l'acceptation de la contraception est en relation avec l'âge? L'amélioration des performances dans le travail est-elle liée à la formation? L'élévation du niveau de la connaissance de la contraception est-elle liée aux messages diffusés par la radio? Le niveau d'utilisation de la contraception est-il fonction du degré d'instruction? Il existe différentes méthodes pour mesurer ces relations comme les coefficients de Pearson ou gamma qui seront présentés plus loin ainsi que d'autres méthodes.

\section{Méthodes d'analyse}

On doit présenter dans le protocole de recherche les différentes méthodes analytiques qui seront utilisées pour dégager les caractéristiques de tendance centrale, la variance, les différences et les relations entre les variables. Les méthodes d'analyse quantitative les plus utilisées dans les études en matière de planification familiale sont les suivantes:
a Transformation des variables
b Analyse univariée
c Analyse des séries chronologiques
d Comparaisons
e Analyse bi-variée
d Analyse multi-variée
g Analyse coût-efficacité
h Analyse de l'efficacité de la contraception
i Analyse de la fécondité

Le protocole de recherche doit comporter des indications sur les méthodes d'analyse à utiliser pour atteindre les objectifs de l'étude. Le choix de la méthode dépend de plusieurs critères dont les plus importants sont premièrement, la pertinence de la méthode choisie pour répondre aux questions soulevées par l'étude, et deuxièmement, le degré de compétence requis pour la compréhension de la méthode et l'interprétation des résultats.

On fournit dans ce qui suit les principales caractéristiques de ces différentes méthodes d'analyse. Cette présentation ne peut constituer en aucun cas un cours d'analyse des données si les méthodes statistiques ne sont pas d'ores et déjà connues. Tous les indicateurs et les tests statistiques mentionnés ci-dessous sont largement utilisés par les chercheurs en sciences sociales. Les manuels de statistique élémentaire en fournissent des présentations plus détaillées. 


\section{a Transformation des variables}

L'analyse des données requiert le plus souvent une transformation de celles-ci. Supposons que l'information contenue dans le questionnaire fournit non pas l'âge de la personne interrogée mais le mois et l'année de naissance. Si l'âge constitue une variable à etudier, il faut, pour l'obtenir, faire la différence entre la date de naissance et la date de l'interview. Une telle opération est plus facile à effectuer pendant la phase d'analyse que pendant le codage grâce à l'ordinateur qui permet une plus grande précision dans les calculs que la méthode manuelle. Ainsi transformée, cette variable peut se prêter à d'autres types de manipulations. Si on veut, par exemple, croiser l'âge avec d'autres variables, il est préférable de limiter la distribution par âge à quelques catégories (groupes de cinq à dix ans), ou même à deux catégories uniquement (par exemple, 15-29 ans et 30 ans et plus). Il existe plusieurs méthodes de transformation des données dont les plus utilisées sont les suivantes:

Le recodage. Au cours de cette opération, les catégories de la variable changent d'intitulés et deviennent moins nombreuses. A titre d'exemple, la transformation d'une distribution par année d'âge en distribution par groupe d'âge de 15-19, 20-24 ans, 25-29 ans.

Le comptage. Si l'information recueillie intéresse l'utilisation passée de chacune des 10 méthodes de planification familiale citées dans le questionnaire, on peut chercher à savoir le nombre de méthodes que chaque femme a déjà utilisées. Dans ce cas, on peut créer la variable "nombre de méthodes déjà utilisées." Le procédé du comptage est utile dans les études qui comportent plusieurs variables différentes.

L'échelle. Plusieurs techniques d'échelles sont utilisées dans les recherches opérationnelles en matière de planification familiale dont la plus connue est la technique de Likert utilisée parfois pour étudier les attitudes. La méthode consiste à présenter plusieurs propositions à l'enquête reflétant diverses attitudes possibles (exemple: la pilule est cancérigène, la pilule est très efficace, la pilule est d'utilisation facile, la pilule provoque des malaises). La personne enquêtée doit répondre pour chacune des propositions si elle est tout à fait d'accord, modérément d'accord ou pas du tout d'accord. Chaque réponse est notée entre 1 et 5 ; la note 1 correspondant à une attitude très favorable et la note 5 à une attitude très defavorable (ou vice-versa). Le score indiquant l'attitude globale est obtenue en divisant la somme des notes obtenues aux différentes propositions par le nombre de propositions. On obtient ainsi une note comprise dans l'échelle allant de 1,0 à 5,0.

Transformations conditionnelles. Les transformations conditionnelles sont utilisées lorsque la transformation d'une variable dépend d'une autre. Supposons qu'on pose les trois questions suivantes: (1) Combien avez-vous d'enfants? (2) Voulez-vous en avoir d'autres? (3) Si oui, combien voulez-vous d'enfants supplémentaires? Dans ce cas, on peut créer une nouvelle variable appelée "nombre d'enfants souhaités" qui est obtenue en additionnant le nombre actuel d'enfants (à partir de la question 1 et le nombre d'enfants supplémentaires souhaités (à partir de la question 3). Cette addition n'est possible que si la réponse à la question 2 est "oui."

Autres transformations mathématiques. Le calcul de l'âge à partir de la date de naissance et la date d'interview est un exemple de transformation mathématique. Un autre exemple, dans le cadre des analyses au niveau de la communauté est le taux d'utilisation de la contraception obtenu en divisant le nombre d'utilisatrices par l'effectif des couples concernés.

\section{b Analyse univariée}

Comme son nom l'indique, ce type d'analyse s'intéresse à des variables isolées. Il est souvent important d'étudier les fréquences au niveau d'une seule variable. Ainsi la structure par âge d'une population échantillon peut être comparée à celle de la population mère afin de s'assurer du degré de représentativité de l'échantillon étudié. On peut aussi dégager à partir de l'échantillon certaines données importantes à étudier, inédites jusqu'alors, et qu'il est possible de généraliser pour la population mère. Il arrive parfois que la majorité des objectifs de l'étude soient atteints par l'analyse univariée. Par exemple, un diagnostic concernant les points forts et les points faibles d'un projet donné peut se faire par une enquête auprès de la clientèle. L'essentiel du diagnostic peut être obtenu en étudiant isolément chacune des réponses aux questions relatives au fonctionnement du projet. 
Le moyen le plus facile pour l'analyse au niveau d'une seule variable est de compter le nombre de cas dans chaque catégorie. Le résultat appelé fréquences de la variable n'est toutefois intéressant que si on effectue certaines opérations statistiques supplémentaires. Ces opérations dépendent quant à elles, du type de variable et plus précisément du type de mesure à effectuer. Pour les recherches opérationnelles en matière de planification familiale, il est utile de distinguer les trois types de mesure correspondant aux variables nominales, ordinales et quantitatives.

Variables nominales. Pour les variables nominales (ou qualitatives), les différentes catégories ne different les unes des autres que du point de vue nomenclature. En d'autres termes, l'une des catégories de la variable n'est pas nécessairement plus ou moins élevée, ni plus ou moins grande qu'une autre catégorie. La seule différence réside au niveau de l'appellation, celle-ci pouvant même être la même. Par exemple, la variable sexe a deux catégories, à savoir, masculin et féminin. Le chercheur peut attribuer le code 1 au sexe masculin et le code 2 au sexe féminin. Ces chiffres ne servent qu'à distinguer les deux catégories l'une de l'autre. On peut aussi bien inverser l'opération et attribuer le code 1 au sexe féminin et le code 2 au sexe masculin. On peut aussi bien attribuer le code 1, 598 au sexe masculin et le code 83 au sexe féminin. Cela ne fait aucune différence. Si on prend un autre exemple, le chercheur peut attribuer différents codes pour désigner les méthodes contraceptives: $1=$ stérilisation féminine; $2=$ stérilisation masculine; $3=$ condom; $4=$ DIU; $5=$ DMPA; 6 = spermicids. Ces chiffres ne servent qu'à distinguer une méthode d'une autre. Un code plus ou moins élevé ne correspond pas du tout à une méthode plus ou moins efficace. N'importe quel chiffre peut servir pour désigner chaque méthode. L'essentiel est de garder le même code pour chaque catégorie.

Les indices obtenus à partir des variables nominales (ou qualitatives) sont parmi les indices les plus limités. Les différentes catégories de la variable nominale sont mutuellement exclusives et ne diffèrent entre elles que du point de vue nomenclature. Les codes attribués aux différentes catégories permettent simplement de regrouper les éléments identiques dans la même catégorie (s'ils ont le même code) et dans des catégories différentes (s'il ont des codes différents). Pour les variables de type nominal, les opérations statistiques possibles sont assez limitées. On peut calculer le mode (la catégorie la plus fréquente). On peut obtenir aussi une répartition proportionnelle. Mais on ne peut pas calculer des moyennes. En effet, on ne peut pas parler du "sexe moyen" ou de "méthode contraceptive moyenne" ou de "religion moyenne" car ces variables sont de type nominal ou qualitatif.

Variables ordinales. Lorsqu'il existe une hiérarchie entre les différentes catégories, on parle de variable ordinale. En d'autres termes, la catégorie ayant pour code 1 peut être considérée comme plus élevée que la catégorie ayant pour code 2, qui peut elle-même être considérée comme plus élevée que la catégorie ayant pour code 3. Par exemple, dans les recherches opérationnelles en matière de planification familiale, il arrive souvent qu'on demande l'opinion des personnes interrogées concernant l'utilisation de la contraception. On peut attribuer un code à chaque type de réponses comme suit:

$$
\begin{aligned}
& \mathbf{1}=\text { Approbation forte } \\
& \mathbf{2}=\text { Approbation modérée } \\
& \mathbf{3}=\text { Approbation faible } \\
& \mathbf{4}=\text { Désapprobation }
\end{aligned}
$$

Les codes attribués aux différentes catégories ne permettent pas seulement de regrouper les éléments semblables (comme c'est le cas pour les variables nominales) mais indiquent aussi une hiérarchie allant de 1, ce qui correspond aux éléments les plus élevés (approbation forte), jusqu'à 4, ce qui correspond aux éléments les moins élevés (désapprobation). Les variables ordinales se prêtent à toutes les opérations statistiques possibles avec les variables nominales comme le mode et les pourcentages. En plus, d'autres types d'opérations sont possibles du fait de la hierarchie entre les différentes catégories comme la médiane, les percentiles et certains tests statistiques presentés plus loin. Mais il n'est pas possible d'obtenir une moyenne, ni un écart-type, ni un coefficient de Pearson. Par exemple, on ne peut pas parler d'attitude moyenne vis-à-vis de la contraception parce que l'écart 
FIGURE 10.1

Variable ordinale: Opinion

relative à la contraception

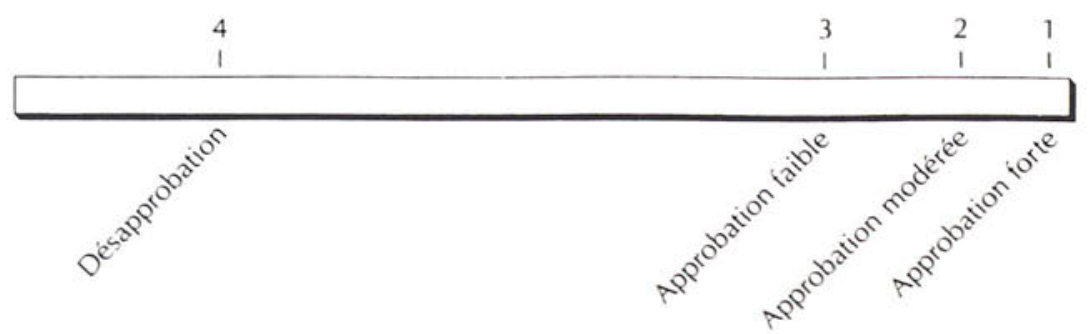

entre chaque catégorie n'est pas connu. Dans l'exemple précédent, on ne sait pas si l'écart entre la catégorie 1 (approbation totale) et la catégorie 2 (approbation modérée) est le même qui existe entre la catégorie 4 (desapprobation). Il se peut même que les réponses classées parmi les catégories 1,2 , et 3 soient plus proches les unes des autres que ne le sont les réponses classées parmi les catégories 4 qui peuvent être très différentes entre elles. La figure 10.1 illustre ce type de situation. Il existe une hiérarchie mais l'écart n'est pas le même entre les differentes catégories.

Variables quantitatives. Une variable quantitative est celle dont la distance (ou l'écart) entre les différentes catégories est connue. C'est le type même de mesure quantitative. Les codes attribués aux différentes catégories possèdent toutes les caractéristiques des variables nominales ou ordinales avec en plus une valeur constante entre les différentes catégories qui sont également espacées. L'âge est une variable quantitative, de même que la parité, la durée de pratique contraceptive et la date d'acceptation d'une méthode de planification familiale. Pour chacune de ces variables, la distance entre une catégorie et celle qui suit est connue et elle est constante. Une personne âgée de 34 ans est une personne plus vieille de 4 ans qu'une personne âgée de 30 ans. Une personne de parité 6 a deux enfants de plus qu'une personne de parité 4 . Le temps, la température, l'argent et le poids sont des variables quantitatives parce que la distance entre les catégories est connue et elle est constante. Une telle situation est representée sur la figure 10.2. Un enfant classé dans la catégorie 5 n'est pas seulement différent (nomenclature) d'un enfant placé dans la catégorie 6 mais il est aussi plus jeune (hiérarchie) et il est plus jeune d'une année (distance). Toutes les opérations statistiques effectuées sur les variables nominales ou ordinales sont possibles aussi avec les variables quantitatives sans être toujours forcément appropriées pour ces dernières. Les variables quantitatives peuvent se prêter, en plus, à des calculs de moyenne, d'écart-type, de coefficient de Pearson ainsi que d'autres indices statistiques.

\section{c Analyse des séries chronologiques}

L'analyse peut se révéler à la fois très simple et très révélatrice si elle a pour objet l'étude de séries statistiques chronologiques. L'analyse consiste dans ce cas à suivre l'évolution d'un indice statistique dans le temps. Cet indice peut se présenter sous différentes formes telle qu'une fréquence (exemple $=$ nombre d'acceptrices par mois), un pourcentage (exemple $=$ un taux de prévalence) ou une caractéristique de tendance centrale (exemple $=$ taille familiale moyenne desirée). Si un nouvel élément est introduit au cours de la période d'observation (exemple: $\mathbf{O}_{1} \mathbf{O}_{2} \mathbf{O}_{3} \mathbf{O}_{4} \mathbf{X} \mathbf{O}_{5} \mathbf{O}_{6} \mathbf{O}_{7} \mathbf{O}_{8}$ ), on peut en mesurer l'impact en comparant les tendances avant et après comme on le voit dans le tableau 10.1 .

Un examen rapide de cette série permet de voir qu'un élément particulier a dû se produire au cours du mois de mai. Si cet élément consiste en une vaste campagne nationale en faveur de la stérilisation masculine, on peut voir qu'elle a eu un impact impressionnant. Mais ce tableau nous montre aussi que le nombre de stérilisations a très vite chuté après la campagne, passant d'une moyenne mensuelle de 116 avant la campagne à une moyenne mensuelle de 55 après.

Ce tableau peut se prêter à plusieurs types d'interprétations. L'une de ces interprétations est l'anticipation. En d'autres termes, certaines personnnes qui auraient dû avoir leur opération en juin, juillet, août, etc. ont pu décider de la faire pendant le mois de mai à l'occasion de la campagne

FIGURE 10.2

Variable quantitative d'âge

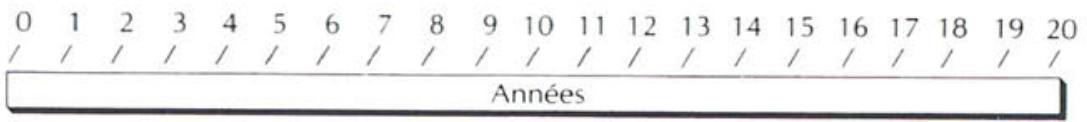


TABLEAU 10.1 Série chronologique: Effectifs mensuels de stérilisations masculines réalisées en 1982

\begin{tabular}{|c|c|c|}
\hline Mois & Nombre & \\
\hline Janvier & 1207 & \multirow{8}{*}{ Moyenne janvier-avril $=116$} \\
\hline Février & 110 & \\
\hline Mars & 130 & \\
\hline Avril & 105 & \\
\hline Mai & 1.200 & \\
\hline Juin & 307 & \\
\hline Juillet & 45 & \\
\hline Août & 15 & \\
\hline Septembre & 30 & \multirow[t]{4}{*}{ Moyenne juin-décembre $=155$} \\
\hline Octobre & 60 & \\
\hline Novembre & 90 & \\
\hline Décembre & $115]$ & \\
\hline Total & 2.050 & \\
\hline
\end{tabular}

d'incitation. Une deuxième interprétation possible consiste à penser que le personnel de planification familiale comme les médecins, les infirmiers et le personnel de terrain, ont réduit leurs activités de motivation après l'effort déployé pendant le mois de mai et n'ont pas pu, simplement, recruter autant d'accepteurs qu'avant. Les fréquences présentées dans le tableau ne font que suggérer de telles interprétations mais ne les prouvent pas. Ces différentes interprétations possibles peuvent constituer le fondement d'une étude concernant la baisse du nombre de stérilisations observée après le mois de mai.

\section{d Comparaisons}

La comparaison de certaines variables fait souvent partie des objectifs de recherche. Tel est le cas pour les recherches expérimentales dans lesquelles les résultats les plus importants se dégagent par la comparaison du groupe expérimental avec le groupe témoin. Si on mène une recherche expérimentale pour étudier l'effet de l'introduction d'un programme donné sur la pratique contraceptive, on peut procéder à la comparaison des données relatives au groupe experimental à celles du groupe témoin. Si on suppose que les deux groupes ont été tout à fait comparables au depart et que les modifications qui risquent d'intervenir par la suite ne peuvent être imputables qu'à l'introduction du nouveau programme, on peut avancer que l'observation d'une différence significative entre les deux groupes constitue un succès du programme.

Pour comparer deux à deux les moyennes entre elles on utilise le plus souvent le test $t$. Ce test est employé pour les échantillons de faibles effectifs ( $N$ égal ou inférieur à 30 ) avec l'hypothèse que les unités étudiées sont tirées d'une distribution normale avec une même variance. Pour comparer les pourcentages, on utilise le plus souvent le test khi-carré $\left(\mathbf{X}^{2}\right)$. On voudrait parfois comparer les pourcentages obtenus à deux moments différents pour le même échantillon. Dans un tel cas, le test le plus approprié est le test de McNemar des modifications significatives.

Si on doit comparer plus que deux moyennes entre elles à la fois, la technique la plus appropriée est celle de l'analyse de variance (ANOVA) qui emploie le test $\boldsymbol{F}$ pour mesurer les degrés de signification des résultats.

\section{e Relations bi-variées}

Les objectifs des recherches opérationnelles en matière de planification familiale exigent souvent l'étude des relations des paires de variables et notamment entre les indices à l'introduction du programme et les indices liées aux effets attendus. Les types de techniques à adopter pour l'étude des relations bi-variées dépendent de la nature des variables si elles sont nominales, ordinales ou quantitatives.

Relations entre variables nominales. La première démarche à faire pour l'étude des relations entre variables nominales consiste à effectuer le croisement préalable de deux variables. Le test du khi-carré peut alors être employé pour déterminer le degré de signification de la relation qui se dégage. Ce test ne mesure pas toutefois l'importance des relations. Pour ce faire, le calcul "d'indices 
d'association" est nécessaire et l'une des methodes pour les variables nominales est le V de Cramer, derivé du khi-carré.

Relations entre variables ordinales. Il existe plusieurs types d'indices d'association pour étudier le croisement de variables ordinales. Le plus facile à calculer est l'indice gamma pour lequel il n'existe malheureusement pas de test de signification facile à utiliser (le test du khi-carré peut être utilisé pour verifier le degré de signification de la relation entre les variables, mais il ne faut pas oublier leur caractère ordinal, ce qui enlève à ce test son efficacité pour le cas de gamma).

Relations entre variables quantitatives. Les relations entre les variables quantitatives peuvent être analysées avec ou sans croisement préalable. Si les variables sont croisées, le type de relation devient apparent et on peut calculer les valeurs de gamma ou du V Cramer. Mais il est plus courant de mesurer les relations entre les variables quantitatives sans les croiser et ceci à l'aide du coefficient de corrélation de Pearson $(r)$. Le degré de signification de $r$ peut être effectué par le test $t$.

La relation peut être étudiée par l'analyse de regression linéaire si on distingue les variables dépendantes des variables indépendantes. Dans ce cas, c'est le coefficient de regression d'ordre zéro qui indique la variation moyenne de la variable dépendante, associée à la variation d'une unité de la variable indépendante.

Les relations établies avec les indices de regression et de correlation sont de type linéaire, et dans ce cas, la variation de l'une des variables vers la hausse ou vers la baisse est une fonction directe de l'augmentation ou de la diminution de l'autre variable.

Ces indices ne sont pas sensibles aux variations non linéaires, pour lesquelles les valeurs de l'une des variables sont associées aussi bien aux valeurs élevées qu'aux valeurs faibles de l'autre variable (mais non pas aux valeurs médianes).

Tous les indices d'association présentés ci-dessus, à l'exception du coefficient de regression et de l'indice $V$ de Cramer, prennent des valeurs comprises entre -1,00 (relation parfaitement negative) et $+1,00$ (relation parfaitement positive). Lorsqu'il n'existe aucune relation entre les variables, le coéfficient est égal à 0,00 .

On doit remarquer que ces indices ne sont pas de type causal. Si on a trouvé que les personnes ayant lu un document sur le DIU sont celles qui utilisent le plus fréquemment de DIU, cela ne veut pas dire nécessairement que c'est la lecture du document qui a causé l'utilisation du DIU. Il se peut très bien que les personnes ayant accès au DIU soient les mêmes personnes ayant un accès à la documentation et la relation qui s'est dégagée soit tout simplement une coïncidence. Il arrive même qu'une relation est réellement de type causal mais dans un sens contraire à celui dégagé par la relation. Par exemple, on peut fournir la documentation à toutes les nouvelles utilisatrices de DIU pour les informer plus en détail des caractéristiques de leur nouvelle méthode ou bien, elles peuvent demander elles-mêmes une telle documentation après l'insertion du DIU, surtout si certains effets secondaires commencent à se faire sentir. Dans ce cas, il éxiste au moins trois types de relations possibles comme le montre la figure 10.3 .

FIGURE 10.3

Trois relations possibles entre I'accès à la documentation et I'utilisation du DIU
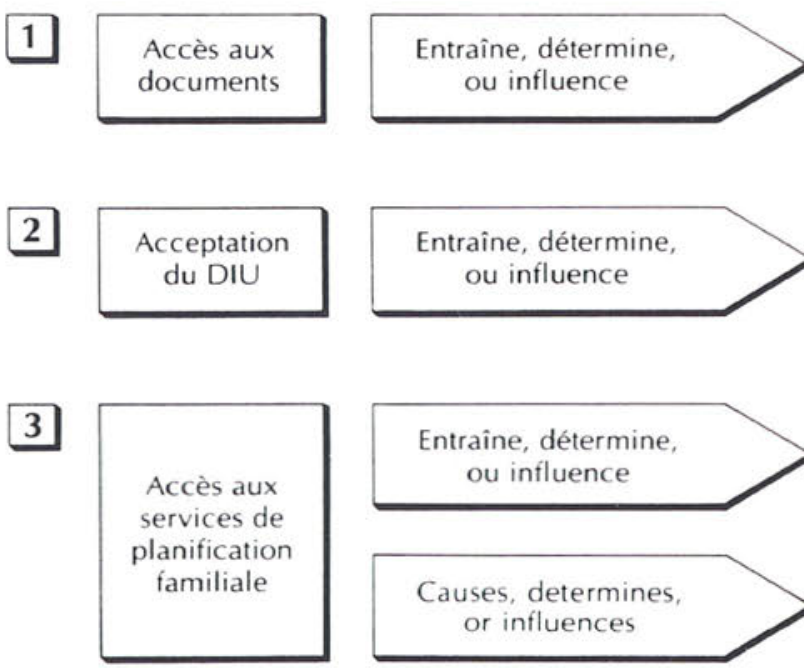
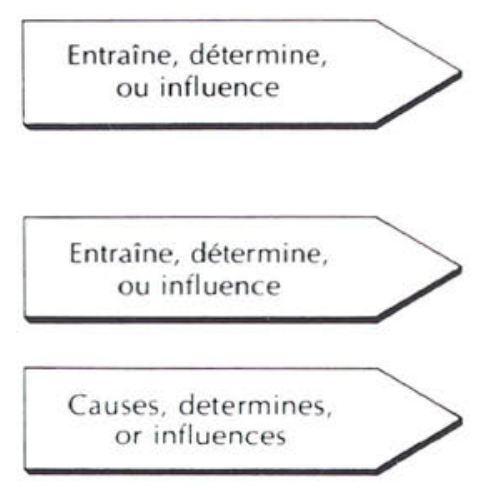

Acceptation du DIU

Accès aux documents

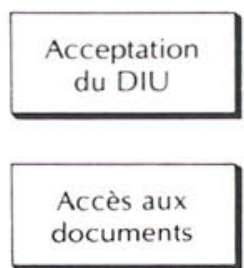




\section{f Analyse multivariée}

L'analyse des relations entre deux variables uniquement soulève parfois des interrogations que seule l'analyse multivariée peut résourdre. Par exemple, si une relation semble se dégager entre la religion et l'utilisation de la contraception, on peut penser à priori que les différences observées sont le signe d'une relation causale directe. On peut toutefois penser aussi que les différences sont dues à d'autres facteurs. Par exemple, on a remarqué depuis longtemps aux Philippines que la communauté musulmane a tendance à moins utiliser la contraception que la majorité de la communauté chrétienne. Cette relation n'est pas toutefois de type causal, car la communauté musulmane vit en général dans les régions les moins pourvues en personnel et en centres de planification familiale et se caractérise aussi par un statut socio-économique relativement bas. Pour vérifier si la relation entre la religion et la pratique contraceptive s'explique par les différences existantes en matière de statut socio-économique et d'accès aux services de planification familiale, on doit contrôler ou maintenir constantes les variables liées au statut et à l'accès; si la relation demeure quand même inchangée, on peut conclure que les variables liées au statut et à l'accès n'ont pas d'influence, sinon très peu. Si au contraire, la relation disparaît, on peut conclure que c'est une fausse relation provenant des effets simultanés des variables dépendantes et indépendantes sur les variables de contrôle.

Les techniques qui permettent au chercheur de maintenir constantes une ou plusieurs variables sont appelées des analyses multivariées car elles font intervenir plusieurs variables (plus de deux). La plupart des techniques multivariées permettent également de mesurer le degré d'association entre une variable dépendante et deux (ou plus) variables indépendantes simultanément. Il existe plusieurs techniques différentes dont l'utilisation dépend du type de variables à étudier. Pour les variables dépendantes quantitatives, il existe trois possibilités dont le choix est fonction de la nature des variables indépendantes.

Toutes les variables sont quantitatives. Lorsque les variables dépendantes et indépendantes sont toutes des variables quantitatives, la technique la plus appropriée est celle de l'analyse de régression multiple. C'est la plus commune et la plus utilisée des techniques multivariées. Elle engendre des coefficients de régression partielle qui indiquent le degré d'augmentation de la variable dépendante en fonction de l'augmentation d'une unité de chacune des variables indépendantes, tout en maintenant constantes les autres variables indépendantes. Ces coefficients sont souvent convertis en coefficients de régression normalisés (beta) qui indiquent le degré de variation de la variable dépendante en fonction des variations de la variable dépendante en fonction des variations de la variable indépendante en termes d'écart-type plutôt que d'unité de mesure. Cette technique engendre aussi un coefficient de régression multiple $(R)$, qui résume les relations simultanées entre la variable dépendante et toutes les variables indépendantes. Le carré du coefficient de régression multiple, connu comme le coefficient de détermination $\left(R^{2}\right)$, mesure la proportion de la variance de la variable dépendante expliquée par toutes les variables indépendantes prises ensemble.

Variables quantitatives et de catégorie. Lorsque la variable dépendante est une variable quantitative alors que les variables indépendantes sont des variables de catégorie (nominales ou ordinales), la technique la plus appropriée est celle de l'analyse de régression multiple avec variables binaires - une variable binaire est une variable qui ne comporte plus que deux catégories. Une variable de catégorie peut être convertie en plusieurs variables binaires-par exemple, une variable nominale à trois catégories représentant la religion (chrétiens, musulmans, Hindous) peut être convertie en trois variables de deux catégories chacune. On peut créer la variable "chrétiens" en réservant le code 1 à tous les chrétiens et le code 0 à tous les autres; de même, on peut créer la variable "Musulmans" en réservant le code 1 aux musulmans et le code 0 à tous les autres; et enfin, on peut créer la variable "Hindous" en réservant le code 1 aux hindous et le code 0 à tous les autres. De la même façon une variable à quatre catégories peut être transformée en quatre variables ayant chacune deux catégories et une variable à cinq catégories en cinq variables-de deux catégories chacune et ainsi de suite. Pour l'analyse de régression multiple, toutes les variables ainsi obtenues sauf une sont prises comme variables indépendentes, et les coefficients de régression ainsi calculés indiquent l'impact différentiel au niveau de la variable dépendante de la catégorie prise en compte 
et des catégories laissées de côté. L'interprétation de $R$ et $R^{2}$ est la même que dans une analyse de régression ordinaire.

Toutes les variables sont des variables de catégorie. Lorsque la variable dépendante est une variable quantitative et les variables indépendantes sont toutes de catégorie, la technique la plus appropriée est l'analyse de classification multiple (Multiple Classification Analysis: MCA). Cette technique est une véritable extension de l'analyse de régression multiple aux variables transformées en variables binaires, mais elle fournit une valeur Beta à chacune des catégories d'origine de la variable, alors que l'analyse de régression produit moins de valeurs intéressantes pour les variables à étudier. L'analyse MCA produit aussi des valeurs $R$ et $R^{2}$.

Si la variable dépendante est une variable de catégorie, l'analyse se limitera en général à un croisement multidimentionnel des variables. Si toutes les variables indépendantes ou certaines d'entre elles sont quantitatives, elles peuvent être facilement transformées en variables de catégorie). Au cours des dernières années, on a pu mettre au point certaines techniques plus complexes d'analyse multivariée utilisant des variables dépendantes nominales, mais il n'y a que quelques chercheurs qui savent bien les utiliser et les programmes informatiques correspondants ne sont pas encore largement disponibles.

Les techniques d'analyse multivariée sont des instruments d'analyse très puissants mais que l'on doit utiliser avec prudence. Elles reposent sur des hypothèses dont certaines ne sont pas toujours verifiées, ce qui rend certains résultats moins crédibles. Le protocole de recherche ne doit comporter une analyse multivariée que si la technique est bien acquise ou s'il existe une assistance adéquate en la matière.

\section{g Les analyses coûts-efficacité}

Tous les programmes de planification familiale exigent des moyens financiers pour mener des activités et obtenir les resultats souhaités. Dans la plupart des programmes de planification familiale plusieurs activités peuvent viser le même objectif en termes de naissances évitées de nouveaux clients ou d'années-couples de protection. Tous les administrateurs peuvent se poser logiquement cette question "Quelle est l'activité qui coûte le moins cher?" Les analyses en termes de coûtsefficacité (ACE) peuvent répondre à ce type de question. Ces analyses constituent surtout des instruments de décision, ce qui n'a de sens que lorsqu'il est possible de comparer les coûts relatifs de deux approches différentes ou plus, ou bien l'évolution d'une seule approche dans le temps. Ces analyses permettent d'examiner différentes alternatives possibles pour atteindre l'objectif final en fonction du coût.

Les analyses coûts efficacité sont basées sur un rapport ayant comme numérateur les coûts et comme dénominateur des résultats du programme. Bien que les calculs soient simples, l'ACE présente toutefois certaines difficultés. Il faut pouvoir, d'abord, déterminer les coûts précis du programme, ce qui n'est pas toujours facile, surtout si les activités de planification familiale sont complètement intégrées aux autres activités de santé. Par exemple, quelle proportion du budget des cliniques où sont éffectuées des insertions de DIU, est imputable au programme de planification familiale? La même question se pose pour le temps de travail (et donc d'une partie des salaires) consacré par les médecins et infirmiers à la planification familiale. Doit-on tenir compte aussi des frais généraux ainsi que des autres types de coûts indirects? De même, doit-on tenir compte de l'inflation et des fluctuations des cours de la monnaie? Ces questions et bien d'autres se posent inévitablement dans les analyses coûts-efficacité.

En plus de cette difficulté liée à la détermination du coût, il en existe une deuxième liée aux données relatives à l'effet du programme. Il s'agit de préciser les unités de mesure comme la clientièle, l'année-couple de protection, ou la naissance évitée et ce que ces termes signifient concrètement. D'autres difficultés existent encore-les effets de certains programmes, par exemple, sont tributaires du temps. La stérilisation peut avoir des effets pendant plusieurs années. Le calcul du rapport coûts-efficacité doit être basé sur des hypothèses concernant le facteur temps. Parfois, ce type d'analyse fait intervenir un nombre d'hypothèses tellement important qu'on arrive à douter de leur utilité en matière de décision. De plus, il peut arriver que le programme le plus indiqué du point de vue coûts-efficacité ne soit pas forcément le plus indiqué du point de vue politique et social. 
Les analyses coûts-efficacité sont plus pertinentes quand il s'agit de comparer des programmes précis et limités dans le temps, avec des objectifs clairement définis et des budgets détaillés. Supposons, par exemple, qu'on définisse les résultats en terme de continuité dans l'utilisation de nouvelles acceptrices de pilules et de continuité dans l'utilisation pendant au moins une année. On peut mener une analyse coûts-efficacité pour voir si ce sont les équipes mobiles, le personnel de terrain ou les cliniques qui sont les plus efficaces du point de vue de leur coût relatif. Le même genre d'analyse peut être effectué pour décider du type de formation le plus apte à fournir du personnel de terrain. De même, on peut comparer les coûts-efficacité relatifs des opérations de stérilisation effectuées dans un camp mobile ou bien dans une clinique ou dans un hôpital.

\section{h Efficacité de la contraception}

Il est souvent important dans les recherches opérationnelles en matière de planification familiale d'étudier la probabilité qu'une nouvelle acceptrice continue, mois après mois, à utiliser une méthode et ne tombe pas enceinte. Ces probabilités peuvent être obtenues par une analyse de l'efficacité de contraception à l'aide des tables de survie. Les indices les plus importants fournis par ce type d'analyse sont les taux de continuation et les taux de grossesse. Des distinctions peuvent être faites entre taux de continuation mensuels et cumulés, entre les taux de continuation d'une méthode et les taux de continuation pour l'ensemble des méthodes, entre les taux bruts et les taux nets de grossesse, et enfin, entre les grossesses accidentelles et l'ensemble des grossesses.

Un taux mensuel de continuation mesure la probabilité pour une personne ayant commencé le mois en utilisant une méthode de continuer à l'utiliser jusqu'à la fin du mois. Un taux de continuation cumulé mesure la probabilité qu'une acceptrice soit toujours utilisatrice de la méthode un certain nombre de mois après l'acceptation.

Un taux de continuation d'une méthode mesure la probabilité de continuer l'utilisation de la même méthode.

Un taux de continuation de toutes les méthodes mesure la probabilitié de continuer l'utilisation de la contraception qu'elle que soit la méthode.

Un taux brut de grossesse mesure la probabilité pour une femme de tomber enceinte pendant une periode donnée sans tenir compte des risques d'abandon (comme l'expulsion de DIU, ou en raison des effets secondaires).

Un taux net de grossesse mesure la même probabilité que le taux brut de grossesse mais en tenant compte des risques d'abandon.

Le taux d'échec mesure la probabilité pendant une période donnée de tomber enceinte tout en utilisant une méthode contraceptive.

Le taux global de grossesse mesure la probabilité pendant une période donnée après l'acceptation d'une méthode de tomber enceinte compte tenu de l'utilisation ou de la non utilisation de la méthode au moment de l'observation.

\section{i Analyse de la fécondité}

Comme la plupart des programmes de planification familiale visent à susciter ou à accélérer une baisse de la fécondité, les recherches opérationnelles de planification familiale doivent souvent inclure des analyses sur la fécondité. A cet effet, les indices de fécondité les plus utiles sont les suivants:

Taux brut de natalité: nombre annuel de naissances pour 1.000 habitants.

Taux global de fécondité générale: nombre annuel de naissances pour 1.000 femmes en âge de procréer.

Taux global de fécondité légitime: nombre de naissances pour 1.000 femmes mariées en âge de procréer.

Taux de fécondité générale par âge: nombre de naissances pour 1.000 femmes appartenant à un groupe d'âge donné.

Taux de fécondité légitime par âge: nombre de naissances pour 1.000 femmes mariées appartenant à un groupe d'âge donné.

Somme des naissances réduites: nombre moyen d'enfants qu'aurait une femme pendant toute sa période de reproduction suivant une loi de fécondité donnée. 
FIGURE 10.4

Exemple d'un tableau fictif:

Prévalence de la

contraception selon les

disponibilités en personnel

(en pourcentage)

\begin{tabular}{|c|c|c|c|}
\hline \multirow[b]{2}{*}{$\begin{array}{l}\text { Prévalence de la } \\
\text { contraception }\end{array}$} & \multicolumn{3}{|c|}{$\begin{array}{l}\text { Disponibilités en personnel de santé } \\
\text { et de planification familiale dans le village }\end{array}$} \\
\hline & $\begin{array}{l}\text { Non } \\
(N=)\end{array}$ & $\begin{array}{l}\text { Oui } \\
(N=)\end{array}$ & $\begin{array}{c}\text { Ensemble } \\
(N=)\end{array}$ \\
\hline \multicolumn{4}{|l|}{$50+$ pourcent } \\
\hline \multicolumn{4}{|l|}{$25-49$ pourcent } \\
\hline \multicolumn{4}{|l|}{ o-24 pourcent } \\
\hline Total & 100 & 100 & 100 \\
\hline
\end{tabular}

\section{Tabulation fictive}

La construction des tableaux fictifs permet de visualiser la manière dont seront présentées les données. Un tableau fictif comporte tous les éléments d'un tableau réel avec les données en moins, car les cases sont vides (voir la figure 10.4). Les principales relations qui devront être étudiées seront présentées dans le protocole de recherche sous forme de tels tableaux fictifs.

Les données qui résultent du croisement de deux variables sont en général présentées sous forme de pourcentages calculés sur le total en ligne ou en colonne. Lorsqu'une variable dépendante est croisée avec une variable indépendante, les pourcentages doivent être calculés de manière à obtenir 100 pour 100 pour chaque catégorie de la variable indépendante. Par exemple, dans la figure 10.4 , le total en pourcentage est calculé pour chaque catégorie de la variable indépendante (dans ce cas, la disponibilité du personnel).

\section{En résumé-plan d'analyse des données}

1 Décrire en détail chacune des techniques d'analyse qui seront utilisées en indiquant le rôle de chacune pour la réalisation des objectifs de l'étude, le type de variables concernées et enfin, les raisons qui ont déterminé ces choix.

2 Présenter sous forme fictive les tableaux les plus importants.

3 Le plan d'analyse doit montrer clairement comment les objectifs de l'étude vont être atteints, comment chaque variable va être utilisée et enfin comment chaque hypothèse va être testée. 


\section{Diffusion des résultats}

Une recherche n'a d'intérêt que si les résultats sont communiqués sous une forme compréhensible et accessible à d'autres personnes concernées. La recherche opérationnelle a été définie au début de ce manuel comme un processus comprenant cinq étapes fondamentales. Les deux dernières étapes sont constituées par la diffusion et l'utilisation des résultats. Le processus de la recherche opérationnelle n'est pas complet si l'on n'apporte pas à ces deux dernières étapes autant de soin et d'attention qu'aux trois étapes précédentes.

\section{A Stratégie de diffusion}

Il faut toujours garder à l'esprit le fait qu'une recherche opérationnelle vise avant tout à fournir les informations nécessaires aux directions administratives et aux responsables des programmes afin de leur permettre d'effectuer les modifications appropriées pour améliorer les prestations de service. Il est donc recommandé de mettre au point une stratégie de diffusion si l'on veut qu'une recherche opérationnelle ait un impact maximum. Cette stratégie doit répondre à ces trois questions essentielles:

1 Qui sont les utilisateurs potentiels des résultats de cette recherche opérationnelle?

2 Quels sont les résultats qui seront particulièrement utiles pour chacune des catégories des utilisateurs potentiels?

3 Quelles sont les voies médiatiques les plus indiquées pour atteindre chacune de ces catégories d'utilisateurs?

Dans la plupart des cas, le public ou les utilisateurs des résultats d'une recherche opérationnelle se grouperont en catégories allant des plus hauts responsables aux utilisateurs des services en passant par les administrateurs du programme, la communauté scientifique, les cadres moyens et le personnel de terrain. Il faudra donc adapter le contenu du message (résultats) au public ciblé. Une partie des résultats présentera parfois plus d'intérêt pour une catégorie de public que pour une autre; et certains moyens de communication seront plus appropriés pour une catégorie que pour d'autres, ce qui rend la tâche encore plus complexe. En d'autres termes, l'objectif essentiel d'une stratégie de diffusion est d'identifier la/les voie(s) médiatique(s) les plus efficaces pour faire connaître les résultats les mieux adaptés aux besoins des différentes catégories du public (utilisateurs). D'une manière générale, une bonne stratégie comprendra l'utilisation répétée de différentes voies médiatiques durant une certaine période, et ce pour atteindre une audience la plus large possible.

\section{B Rédaction des rapports de recherche}

Les rapports de recherche doivent être publiés au moins sous deux formes différentes, sinon plus. Le style de chaque rapport doit être adapté au public visé.

Si l'audience visée est constituée de responsables du programme, on doit garder à l'esprit 
que ces responsables ne sont pas des chercheurs versés dans les procédés méthodologiques. Les informations techniques relatives à l'échantillonnage, le procédé de recherche adopté ou à tel autre aspect méthodologique ne doivent pas alourdir le rapport et masquer les résultats les plus importants. Les documents techniques peuvent toujours être ajoutés dans les annexes, plutôt que d'être inclus dans le rapport même.

Il arrive que les responsables de programme aient des difficultés à réaliser les répercussions des résultats de recherche sur la gestion du programme. Le rapport doit donc indiquer les modifications suggérées par les résultats de recherche pour l'amélioration du programme.

Les responsables de programme se plaignent souvent du fait que les rapports de recherche sont inutilement compliqués et qu'ils sont publiés si tardivement que leur contenu est dépassé au moment de leur publication. Afin d'éviter ces désagréments, il est préférable de publier des rapports préliminaires dès que certains résultats intéressants sont connus. Rédiger un sommaire du rapport final. Dans ce sommaire, il est inutile et maladroit d'indiquer qu'on a utilisé un procédé de recherche quasi-expérimental avec des groupes témoin non identiques pour obtenir des données qui ont été analysées en utilisant l'analyse de classification multiple. Il est au contraire recommandé de mettre l'accent sur les résultats les plus intéressants pour l'amélioration du programme. Utiliser pour la présentation des résultats, des graphiques simples et faciles à interpréter. Enfin, rédiger le rapport dans un langage clair en évitant le jargon spécifique à la recherche.

Si l'on souhaite atteindre les chercheurs et la communauté scientifique, la forme du rapport sera tout à fait différente de celle du rapport destiné aux responsables du programme. La lecture de ce rapport doit permettre aux personnes intéressées d'apprécier la valeur scientifique de l'étude ainsi que la pertinence du choix méthodologique; d'autre part, ce rapport doit leur permettre de refaire, si elles le désirent, une étude semblable pour d'autres régions ou d'autres thèmes de recherche.

\section{Exemple des principaux chapitres d'un rapport final}

I Page de garde (titre, auteurs, organisme de tutelle, date)

II Préface (remerciements, origine du financement)

III Sommaire

IV Généralités (lieu d'étude, conditions)

V Présentations des publications existantes

VI Aspects méthodologiques (objectifs, hypothèses, recherche, procédés d'analyse, limites de l'étude)

programmes introduits, plan de

VII Résultats

VIII Analyse des résultats

$\mathbf{L}$ Conclusions et recommandations

X Références et Bibliographie

XI Annexes

\section{En résumé —publication des résultats de recherche}

1 Rédiger dans la proposition de recherche un chapitre qui expose la stratégie de diffusion des résultats. Cette stratégie doit répondre aux questions suivantes:

a Qui sont les utilisateurs potentiels des résultats de recherche?

b Quels types de résultats seront les plus intéressants pour quelles catégories d'utilisateurs?

c Quelles sont les voies médiatiques les plus appropriées pour atteindre chaque catégorie concernée? 


\section{Utilisation des résultats de recherche}

L'utilisation des résultats constitue l'objectif de toute étude de recherche opérationnelle. Malheureusement, c'est un objectif qui trop souvent n'est pas tout à fait réalisé. Cette défaillance s'explique d'une part par le fait que les personnes qui mettent au point et réalisent l'étude ne sont pas celles qui utilisent les résultats. D'autre part, il arrive que les chercheurs estiment avoir terminé leur étude une fois que le rapport final a été rédigé et diffusé. Cette conception est erronnée. Comme il a été dit dans le chapitre précédent, le processus de la recherche opérationnelle n'est complet que lorsque les résultats ont été diffusés et que tout a été fait pour en assurer l'utilisation. L'une des étapes fondamentales de la recherche opérationnelle est de veiller à ce que les résultats de l'étude soient utilisés.

Le terme utilisation veut dire tout simplement se servir de "quelque chose". Pour une recherche opérationnelle, ce "quelque chose" peut être soit les résultats de l'étude, soit le processus de recherche. Il y a bien sûr de multiples manières d'utiliser les résultats ou le processus de recherche. Par exemple, les hauts responsables peuvent utiliser les résultats d'une recherche opérationnelle pour élaborer ou bien réorienter la politique de santé publique et de planification familiale. Les directeurs des organismes de prestation de services peuvent utiliser les résultats d'une recherche opérationnelle afin d'adopter les meilleures stratégies de gestion. Ils peuvent aussi se servir du processus de recherche pour identifier les aspects qui posent un problème et orienter les efforts et les resources vers la solution de ces problèmes. De même, les chercheurs peuvent utiliser les résultats des recherches opérationnelles pour affiner leurs concepts en matière de sciences sociales; ils peuvent aussi utiliser le processus mis en place pour l'étude pour développer de nouvelles techniques de collecte de données ou d'analyse. Enfin, le personnel de terrain et les superviseurs peuvent, grâce à ces résultats, évaluer et améliorer leur efficacité en ce qui concerne la qualité des prestations de services.

Il est rare que les résultats d'une recherche opérationnelle soient totalement acceptés et entrainent un remaniement de l'ensemble du système de prestation de services. Il arrive plus fréquemment que les résultats d'une recherche opérationnelle soient associés à d'autres informations (politiques, expériences, opinions des collègues, résultats d'autres recherches) afin d'obtenir un diagnostic plus complet d'une situation. Ces informations supplémentaires peuvent être d'une grande importance, particulièrement si elles fournissent aux responsables un surcroît d'information qui leur permettra de décider en toute connaissance de cause des changements à apporter au système de prestation de services. Dans certains cas, le terme "utilisation" ne se réfère pas aux résultats de l'étude mais au processus de la recherche opérationnelle qui permet d'identifier et d'exposer les problèmes existants, et d'examiner systématiquement les stratégies potentielles capables de résoudre ces problèmes.

Bien qu'un chercheur n'ait pas les moyens de guarantir l'utilisation des résultats de recherche par les responsables, il peut prendre certaines mesures qui favoriseront largement cette utilisation. Par exemple:

1 Identifier dès la conception de l'étude, les responsables qui seront les plus concernés par le sujet. Ces personnes doivent alors être contactées et bien informées des objectifs de l'étude. 
2 Mettre au point un programme visant à impliquer les utilisateurs potentiels des résultats dans toutes les étapes de l'étude. Plus les responsables auront été impliqués dans la programmation, l'exécution et l'analyse d'une étude, plus il seront motivés pour l'utilisation des résultats de recherche.

3 Inclure dans les rapports préliminaires et le rapport final un chapitre "Répercussions de l'étude". Indiquer clairement et brièvement les mesures les plus importantes qui devront être prises à la suite de l'étude.

4 Donner assez de temps aux participants des séminaires organisés au sujet de l'étude pour discuter largement des résultats et développer un programme d'action visant à utiliser ces résultats. Cela peut être fait en divisant les participants en plusieurs groupes.

\section{En résumé-utilisation des résultats de recherche}

Inclure dans la proposition de recherche un chapitre concernant l'utilisation des résultats. Ce chapitre doit:

1 Identifier les organismes jugés les plus concernés par l'étude.

2 Developper la manière dont ces organismes seront impliqués durant les différentes étapes de l'étude telles que la programmation, l'exécution, l'analyse et la diffusion.

3 Indiquer les implications éventuelles au niveau de l'orientation générale ou du fonctionnement du programme. 


\section{Limites de l'étude}

\section{A Limites du projet de recherche}

Aucune étude n'est parfaite et tout projet de recherche se heurte à des difficultés concernant la fiabilité des données, la taille de l'échantillon, la confection du questionnaire ou le plan d'analyse. Quoi que fasse le chercheur pour réduire au maximum ces facteurs, il en subsistera toujours quelques uns; l'important consiste de ne pas les ignorer et de les prendre réellement en compte. Si on choisit, par exemple, un groupe particulier comme échantillon, on doit en tenir compte dans l'analyse et ne pas faire comme s'il était réprésentatif de toute une population. Si, faute de temps et de moyens financiers, on a recours à des sources de données qui peuvent être doûteuses (dossiers cliniques, statistiques de services) il faudra en tenir compte et ne pas faire croire que les données sont parfaites. Si le groupe témoin est relativement différent du groupe expérimental, il ne faudra pas nier l'existence d'un tel aspect. En tout état de cause, il vaut mieux admettre l'existence de telles difficultés et essayer de les résoudre par des méthodes statistiques si possible, ou bien présenter les résultats comme étant provisoires.

\section{B Aspects particuliers}

Tout aspect pouvant avoir une influence sur la qualité de l'étude doit être mentionné. Par exemple: (1) en Asie, il est souvent difficile de mener des travaux de terrain pendant la période des moussons. Dans ce cas, preciser que l'étude ne peut-être programmée qu'en dehors de la période de mousson; (2) si l'on n'a pas accès aux moyens d'informatique, le mentionner; (3) s'il faut attendre la permission officielle pour démarrer l'étude (ce qui exige souvent du temps) il faudra aussi l'indiquer dans le protocole de recherche.

\section{En résumé-limites de l'étude}

1 Relever toutes les limites possibles de l'étude, et les aspects particuliers pouvant avoir une influence sur la qualité des données.

2 Fournir les explications sur les limites de l'étude et les hypothèses pour les dépasser. 


\section{Budget de l'étude}

\section{A Ressources disponibles}

Le protocole de recherche doit contenir des précisions sur les moyens matériels et financiers déjà disponibles pour mener l'étude. On doit preciser, par exemple, l'existence d'enquêteurs et de codeurs expérimentés, des moyens informatiques appropriés, des sources supplémentaires de financement si elles existent, et le temps qui sera consacré par le chercheur principal pour mener l'étude-100 pourcent, 50 pourcent, 10 pourcent. Tous les aspects relatifs aux ressources disponibles, doivent être signalés dans le protocole de recherche.

\section{B Le budget de l'étude}

Le budget doit être conçu d'une manière réaliste. En général, les financiers des projets de recherche ne fournissent pas de fonds pour l'acquisition d'équipements coûteux, ni pour la construction de locaux, ni pour l'achat de véhicules. En ce qui concerne le salaire du chercheur principal, il ne doit pas excéder le niveau déjà perçu par ailleurs. Toute rubrique budgétaire relativement élevée ou inattendue doit être justifiée. Les coûts à chacune des rubriques doivent être définis avec précision en fonction des différentes composantes. Par exemple, les salaires versés aux enquêteurs doivent être présentés comme suit:

Enquêteurs (20 enq. à Rs. 500 par jour x 20 jours)

Le budget doit être présenté selon les grandes catégories de dépenses (voir figure 14.1) et selon l'année si l'étude est programmée pour plus d'un an. Pour des études de longue durée, on peut prévoir une ligne budgétaire qui tienne compte de l'inflation.

\section{En résumé-budget de l'étude}

1 Fournir des précisions sur les ressources déjà disponibles, en indiquant en particulier:

a L'existence de sources supplémentaires de financement, s'il y a lieu.

b La disponibilité des moyens en matière d'informatique, en enquêteurs et en codeurs qualifiés, en secretariat, en vehicules, en bureaux, etc.

2 Le budget de l'étude doit être présenté selon les grandes catégories de dépenses:
a Salaires et indemnités
b Matériel, services informatiques
c Déplacements
d Divers

3 Toute rubrique budgétaire relativement élevée ou inattendue doit être justifiée. 
FIGURE 14.1

Exemple d'un budget de

recherche

Exemple d'un budget de recherche

Baht

$\underline{\text { USS }}$

A Salaires et indemnités

1 Chercheur principal

B2.000/mois X 12 mois $\quad 24.000 \quad 1.200$

2 Chercheur adjoint
B1. $700 \times 12$ mois
$20.400 \quad 1.020$

3 Enquêteurs

20 \& B100/jour $\times 20$ jours

$40.000 \quad 2.000$

4 Codeurs

5 Secrétariat

20 personnes-jours @ B150/jour

(hors budget)

$\underline{\text { Sous-total }}$

87.400

4.370

B Matériel, fourniture, services informatiques

1 Impression du questionnaire

2 Fournitures de bureaux

3 Rédaction/Frappe

4 Programmation informatique

5 Temps ordinateur

6 Imprimerie et envoi du rapport (200 exemplaires)

$3.000 \quad 150$

$7.600 \quad 380$

$4.000 \quad 200$

$5.000 \quad 250$

$15.000 \quad 750$

$\underline{25.000} \quad \underline{1.250}$

Sous-total $\quad 59.600 \quad 2.980$

C Déplacements

1 Pré-test du questionnaire sur le terrain

2 Indemmité journalière de déplacement des enquêteurs (20) \& B100/jour

10.000

500

$\underline{40.000} \quad \underline{2.000}$

Sous-total

50.000

2.500

D Séminaire de diffusion

10.000

500

E Coats divers

2.000

100

Total budgétaire

$\underline{209.000}$

$\underline{10.450}$

Coat du projet

$\underline{\underline{209} .000}$

$\underline{10.450}$

Taux d'échange: Baht $23.000=$ US\$1.00. 


\section{Annexes}

Toutes les informations supplémentaires et pouvant être utiles doivent figurer en annexe du projet de recherche. De telles informations peuvent inclure le curriculum vitae des chercheurs principaux, un exemplaire du questionnaire s'il est déjà prêt, et le formulaire de libre consentement. De même, on peut inclure des documents décrivant l'organisme de tutelle (tel qu'un rapport d'activités annuelles). Enfin, si certaines sources de référence sont citées dans le projet de recherche, on doit les inclure aussi en annexe. 


\section{Page de garde et sommaire}

Bien que situés en tête du document, la page de garde et le sommaire constituent la dernière phase dans l'élaboration du projet de recherche. La page de garde (figure 16.1) fournit les éléments fondamentaux du projet. Un sommaire (figure 16.2) doit contenir un résumé des éléments essentiels de chaque chapitre; il ne doit pas être surchargé par des données inutiles et doit être aussi court et précis que possible (une ou deux pages). Le sommaire doit fournir des renseignements sur les points suivants:

1 Le problème à étudier

2 Les objectifs essentiels de l'étude

3 Les répercussions principales à attendre

4 L'identité du chercheur principal

5 La date de démarrage de l'étude

6 Le lieu de l'étude

7 La méthodologie

8 Les ressources requises

\section{En résumé-page de garde et sommaire}

1 Résumer en une ou deux phrases les éléments essentiels de chaque chapitre.

2 Former un sommaire àpartir des résumés ainsi effectués.

3 Joindre une page de garde au sommaire. 
FIGURE 16.1

Exemple d'une page de garde

\section{Projet de recherche en planification familiale}

1 Titre: Etude expérimentale visant à élever le niveau d'acceptation des méthodes de planification

2 Lieu: Kisumu, Kenia

3 Organisme(s) de tutelle (nom/adresse):

Institut du Développement Rural, Social et Economique (IRSED)

4 Chercheur principal

(nom et adresse):

Dr. Georges Ndeti

Directeur

IRSED

Nairobi, Kenya

5 Début de l'étude: Février, 1991

6 Fin de l'étude: Juillet, 1994

7 Coat total: US\$31.580

Signature

Date 
(Problème) Au Bangladesh, les taux de prévalence de contraception varient beaucoup d'un village à l'autre. Les taux parmi les femmes mariées varient entre un maximum de $80 \%$ et un minimum de $6 \%$, alors que tous les villages bénéficient théoriquement du même niveau de prestation du services de santé et de

(Objectifs) planification familiale. Les objectifs immédiates de l'étude consistent à analyser les facteurs économiques, sociaux et sanitaires qui déterminent les variations régionales de taux de prévalence de la contraception. L'objectif à plus long terme consiste à fournir aux responsables du programme les éléments

(Implications) d'explication nécessaires relatifs à la situation favorable dans certaines régions et défavorable dans d'autres. De tels renseignements permettent l'élaboration de programmes d'éducation adaptés, et de modifier les approches en matière de prestation de services afin de rendre les taux de prévalence plus homogènes dans l'ensemble du pays.

(Qui) L'étude sera menée par l'institut de recherche démographique à (Quand) Dhaka au Bangladesh de Janvier 91 à Decembre 91. Tous les

(Où) villages compris dans deux districts seront classés en fonction de leurs taux de prévalence. Ensuite, on procèdera au tirage

(Méthodes) aléatoire de 25 villages repartis selon les tranches de niveaux de prévalence élevée, moyenne et faible. Dans chacun des villages tirés, on interrogera l'ensemble des femmes mariées âgées de 15-44 ans. La collecte des données intèresse aussi les aspects économiques, sociaux et sanitaires au niveau du village. Une équipe d'enquêteurs formés à cet éffet et de superviseurs

(Ressources) se chargera des activités de terrain. Les travaux de codage, de vérification et de traitment des données se fera sur l'ordinateur de l'université de Dhaka. Le rapport final de recherche est prévu pour Décembre 1992. Le budget nécessaire pour le paiement des salaires du personnel, les déplacements, les questionnaires, le temps d'ordinateur et un séminaire de diffusion s'élève à US\$34.500. 
Les livres de référence (considérés par certains comme des "classiques") sur la méthodologie de la recherche sont énumérées dans la liste ci-dessous. Cette liste comprend aussi une sélection d'études opérationnelles relativement récentes conduites en Asie, en Afrique et en Amérique Latine.

Andrade, S. J., M. G. Shedlin, and E. Bonilla. Mátodos Cualitativos para Evaluación de Programas. Watertown, MA: The Pathfinder Fund, 1987.

Andrews, Frank M., Laura Klem, Terrence N. Davidson, Patrick M. O'Malley, and Willard L. Rodgers. A Guide for Selecting Statistical Techniques for Analyzing Social Science Data. Ann Arbor: Institute for Social Research, 1974.

Andrews, Frank M., James N. Morgan, John A. Sonquist, and Laura Klem. Multiple Classification Analysis (2nd ed.). Ann Arbor: Institute for Social Research, 1975.

Askew, lan. "Organizing Community Participation in Family Planning Projects in South Asia." Studies in Family Planning 20,4, July/August 1989

Barclay, George Watson. Techniques of Population Analysis. New York: Wiley, 1958.

Berelson, Bernard. Content Analysis in Communication Research. New York: Hafner, 1971.

Bertrand, Jane, Roberto Santiso, Stephen H. Linder, and Maria Antonieta Pineda. "Evaluation of a Communications Program to Increase Adoption of Vasectomy in Guatemala." Studies in Family Planning 18, 6/Part I, November/December 1987.

Blalock, Hubert M., An Introduction to Social Research. Englewood Cliffs, New _ Jersey: Prentice-Hall General Sociology Series. 1970.

Causal Models in the Social Sciences. Chicago: Aldine-Atherton, 1971. Social Statistics (2nd ed.). New York: McGraw-Hill, 1972.

Blalock, Hubert M., Jr. and Ann B. Blalock. Methodology in Social Research. New York: McGraw-Hill, 1968.

Blumenfeld, Stewart N. Operations Research Methods: A General Approach to Primary Health Care. Chevy Chase, MD: PRICOR, 1985.

Bogue, Donald J. Cost-Effectiveness Analysis of Family Planning Programs. Manual \#11. Chicago: Community and Family Study Center, 1973

Bongaarts, John. "A Simple Method for Estimating the Contraceptive Prevalence Required to Reach a Fertility Target." Studies in Family Planning 15, 4, July/August 1984.
Bourke, G. J. and J. McGiluray. Interpretation and Uses of Medical Statistics. Oxford and Edinburgh: Blackwell Scientific Publications, 1969.

Bruce, Judith. "Fundamental Elements of the Quality of Care: A Simple Framework." Studies in Family Planning 21,2, March/April 1990.

Campbell, Donald T. and Julian C. Stanley. Experimental and QuasiExperimental Designs for Research. Chicago: Rand McNally, 1963.

Carlson, Ronald H. and Anabel Burgh Crane. "Planning and Managing Useful Evaluations." In Wholey, Newcomer and Associates (eds.), Improving Government Performance. San Francisco: Jossey-Bass, 1989.

Caro, Francis G. (ed.). Readings in Evaluation Research. New York: Russell Sage Foundation, 1971.

Chandrasekaran, C. and Albert I. Hermalin (eds.). Measuring the Effect of Family Planning Programs on Fertility. Dolhain: Ordina Editions, 1976.

Cochran, W. G. and G. M. Cox. Experimental Designs (2nd ed.). New York: Wiley, 1957.

Coeytaux, Francine, Dayl Donaldson, Touhami Aloui, Taoufik Kilani, and Habib Fourati. "An Evaluation of the Cost-Effectiveness of Mobile Family Planning Services in Tunisia." Studies in Family Planning 20, 3, May/June 1989.

Cohen, Jacob. Statistical Power Analysis for the Behavioral Sciences (2nd ed.) Hillsdale, NJ: Lawrence Erlbaum Associates, 1988.

Cook, Thomas D. and Donald T. Campbell. Quasi-Experimentation: Design and Analysis Issues for Field Settings. Chicago: Rand McNally College \& Publishing Co., 1979.

Cuca, Roberto and Catherine S. Pierce. Experiments in Family Planning: Lessons from the Developing World. Baltimore: The Johns Hopkins University Press for the World Bank, 1977.

Davis, James A. Elementary Survey Analysis. Englewood Cliffs, New Jersey: Prentice-Hall, 1971.

Delbecq, Andre L., Andrew H. Van de Ven and David H. Gustafson. Group Techniques for Program Planning. Glenview, IL Scott, Foresman and Co., 1975. 
Delp, P., Arne Thesen, Juzar Motiwalla, and Neelakantan Seshadri. System Tools for Project Planning. Pasitam, 1977.

Dixon, Wilfred J. and Frank J. Mossey, Jr. Introduction to Statistical Analysis. (3rd ed.). New York: McGraw-Hill, 1969.

Dunn, Olive Jean. Basic Statistics: A Primer for the Biomedical Sciences. New York: Wiley, 1964.

Fink, Allene and Jacqueline Kosecoff. An Evaluation Primer. Beverly Hills: Sage Publications, 1978.

Fisher, Andrew A. and Victor de Silva. "Satisfied IUD Acceptors as Family Planning Motivators in Sri Lanka." Studies in Family Planning 17,5, September/October 1986.

Fisher, Andrew A., John Laing, and John Stoeckel. "Guidelines for Overcoming Design Problems in Family Planning Operations Research." Studies in Family Planning 16,2, March/April, 1985.

Fisher, Andrew A. and Raymond Carlaw. "Family Planning Field Research: Balancing Internal Against External Validity," Studies in Family Planning 14,1, January, 1983.

Fitz-Gibbon, Carol Taylor, and Lynn Lyons Morris. How to Calculate Statistics. Beverly Hills: Sage Publications, 1978.

Fitz-Gibbon, Carol Taylor, and Lynn Lyons Morris. How to Design a Program Evaluation. Beverly Hills: Sage Publications, 1978.

Foreit, James and Karen G. Foreit. "Quarterly versus Monthly Supervision of CBD Family Planning Programs: An Experimental Study in Northeast Brazil." Studies in Family Planning 15,3, May/June 1985.

Foreit, James R., James E. Rosen, Miguel Ramos, Eduardo Mostajo, and Rosa Monge. "The Impact of Service Delivery Frequency on Family Planning Program Output and Efficiency." Studies in Family Planning 21,4, July/August 1990.

Freund, John E. Modern Elementary Statistics (2nd ed.). Englewood Cliffs, New Jersey: Prentice-Hall, 1960.

Green, Lawrence W., Marshall W. Kreuter, Sigrid G. Deeds, and Kay B. Partridge. Health Education Planning: A Diagnostic Approach. Palo Alto, CA: Mayfield Publishing Co., 1980.

Green, Lawrence W. and Frances Marcus Lewis. Measurement and Evaluation in Health Education Promotion. Palo Alto, CA: Mayfield Publishing Company, 1986.

Grundy, F., and W. A. Reinke. Health Practice Research and Formal. ized Managerial Methods. Geneva: World Health Organization, 1973.

Guilford, J. P. Psychometric Methods. New York: McGraw-Hill, 1954.

Guttentag, Marcia, and Elmer L. Struening (eds.). Handbook of Evaluation Research (Vols. I and II). Beverly Hills: Sage Publications, 1975 .

Hansen, Morris H., William N. Hurwitz, and William G. Madow. Sample Survey Methods and Theory. New York: John Wiley \& Sons, 1953

Haupt, Arthur and Thomas T. Kane. Population Handbook. Washington, D.C.: The Population Reference Bureau, 1980.

Henerson, Marlene E., Lynn Lyons Morris, and Carol Taylor FitzGibbon. How to Measure Attitudes. Beverly Hills: Sage Publications, 1978

Hermalin, Albert 1. and Barbara Entwisle (eds.). The Role of Surveys in the Analysis of Family Planning Programs. Liege: Ordina Editions, 1980.

Hilton, Elizabeth T. and Arthur A. Lumsdaine "Field Trial Designs in Gauging the Impact of Fertility Planning Programs." In Carl A. Bennett and Arthur A. Lumsdaine (eds.), Evaluation and Experiment: Some Critical Issues in Assessing Social Programs. New York: Academic Press, 1975.

Hyman, Herbert. Survey Design and Analysis. Glencoe: The Free Press, 1955

Jain, Anrudh. "Fertility Reduction and the Quality of Family Planning Services." Studies in Family Planning. 20,1, January/February 1989

Katz, F. M. and R. Snow. Assessing Health Workers' Performance: A
Manual for Training and Supervision. Geneva: World Health Organization, 1980

Katzer, Jeffrey, Kenneth H. Cook, and Wayne W. Crouch. Evaluating Information: A Guide for Users of Social Science Research Reading, Massachusetts: Addison-Wesley Publishing Company, 1978.

Kerlinger, Fred N. Foundations of Behavioral Research. New York Holt, Rinehart and Winston, 1964.

Kish, Leslie. Survey Sampling. New York: Wiley, 1965.

Knox, E. G. (ed.), Epidemiology in Health Care Planning: A Guide to the Uses of a Scientific Method. Oxford: Oxford University Press, 1979

Laing, John. Demographic Evaluation of Family Planning Programs. Canberra: The Australian National University, 1982.

Lewis-Beck, Michael S. Applied Regression: An Introduction. Beverly Hills: Sage Publications, 1980

Lilienfeld, Abraham and David E. Lilienfeld. Foundations of Epidemiology. New York: Oxford University Press, 1980

Miller, Delbert C. Handbook of Research Design and Social Measurement. New York: Longman, 1964.

Morris, Lynn Lyons and Carol Taylor Fitz-Gibbon. Evaluator's Handbook. Beverly Hills: Sage Publications, 1978

Morris, Lynn Lyons and Carol Taylor Fitz-Gibbon. How to Present an Evaluation Report. Beverly Hills: Sage Publications, 1978.

Morton, Richard F. and J. Richard Hebel. A Study Guide to Epidemiology and Biostatistics. Baltimore: University Park Press, 1979

Mosley, Henry W. and Lincoln Chen (eds.), "Child Survival Strategies for Research." Population and Development Review. A Supplement to Vol. 10, 1984

Nie, Norman H., C. Hadlai Hull, Jean G. Jenkins, Karen Steinbrenner, and Dale H. Bent. SPSS: Statistical Package for the Social Sciences (2nd ed.). New York: McGraw-Hill, 1975.

Patton, Michael Quinn. Utilization-FocusedEvaluation. Beverly Hills. CA: Sage Publications, 1978

Qualitative Evaluation Methods. Beverly Hills, CA: Sage Publications, 1980

Payne, Stanley L. The Art of Asking Questions. Princeton, New Jersey: Princeton University Press, 1951.

Peters, Thomas J. and R. H. Waterman, In Search of Excellence. New York: Harper \& Row, 1982.

Phillips, J. F., R. Simmons, G. B. Simmons, and Md. Yunus, "Transferring Health and Family Planning Service Innovations to the Public Sector: An Experiment in Organization Development in Bangladesh." Studies in Family Planning 15,2 March/April 1984

Piotrow, Phyllis, Jose G. Rimon II, Kim Winnard, D. Lawrence Kincaid, Dale Huntington, and Julie Convisser. "Mass Media Family Planning Promotion in Three Nigerian Cities." Studies in Family Planning 21, 5 September/October 1990.

Polansky, Norman A. (ed.). Social Work Research. Chicago: University of Chicago Press, 1970.

Popham, W. James. Educational Evaluation. Englewood Cliffs, New Jersey: Prentice-Hall, 1975.

Population Council. A Manual for Surveys of Fertility and Family Planning: Knowledge, Attitudes, and Practice. New York: The Population Council, 1970

Porntip, Jintaganont, John Stoeckel, and Somsak Butaras. "The Impact of an Oral Rehydration Therapy Program in Southern Thailand." American Journal of Public Health 78,10, October, 1988.

Pressat, Roland. Demographic Analysis. Chicago: Aldine Atherton, 1972

Reynolds, Jack. A Framework for the Selection of Family Planning Program Evaluation Topics. Manual \#1 (rev. ed.). New York: International Institute for the Study of Human Reproduction, 1973 
Operational Evaluation of Family Planning Program Through Process Analysis. Manual $\# 4$ (rev. ed.). New York: International Institute for the Study of Human Reproduction, 1973.

Reynolds, Richard and K. Celeste Gaspari. Cost-Effectiveness Analysis. PRICOR Monograph Series: Methods Paper No. 2 , Center for Human Services, Chevy Chase, MD: May 1985.

Roberto, Eduardo L. Strategic Decision-Making in a Social Program: The Case of Family Planning Diffusion. Lexington, Mass.: Lexington Books, 1975.

Robinson, Warren C. Cost Benefit and Cost-Effectiveness Analysis in Family Planning Programs. In Financial Management of Population/Family Planning Programs. Kuala Lumpur: Inter-Governmental Coordinating Committee, 1976, pp. 8291.

Rosenberg, Morris. The Logic of Survey Analysis. New York: Basic Books, 1968.

Ross, John, J. Donayre, and R. McNamara. "Perspectives on Operations Research." International Family Planning Perspectives 13,4, 1987.

Ross, John, Marjorie Rich, and Janet P. Molzan. Management Strategies for Family Planning Programs. Center for Population and Family Health, School of Public Health, Columbia University, New York, 1989

Rossi, Peter H. and Howard E. Freeman. Evaluation: A Systematic Approach. Beverly Hills: Sage Publications, 1982

Rossi, Peter H. and W. Williams. Evaluating Social Programs: Theory, Practice and Politics. New York: Seminar Press. 1972 .

Ruback, R. B. and C. A. Innes. "The Relevance and Irrelevance of Psychological Research." American Psychologist 43,9, 1988.

Rutman, Leonard. Planning Useful Evaluations: Evaluability Assessment. Beverly Hills: Sage Publications, 1980.

Rutman, Leonard (ed.). Evaluation Research Methods: A Basic Guide, Beverly Hills: Sage Publications, 1977.

Selltiz, Claire, Marie Jahoda, Morton Deutsch, and Stuart W. Cook. Research Methods in Social Relations (rev. 1-volume ed.). New York: Holt, Rinehart and Winston, 1959

Shryock, Henry S. and Jacob S. Siegel and Associates. The Methods and Materials of Demography. New York: Academic Press, 1976. .

Siegel, Sidney. Nonparametric Statistics for the Behavioral Sciences. New York: McGraw-Hill, 1956.

Simon, Julian L. Basic Research Methods in Social Science. New York: Random House, 1969

Sinquefield, Jeanne E. Single and Multiple Decrement Life Table Procedures for Analysis of the Use-Effectiveness of Contraception. Manual 8. Chicago: Community and Family Study Center, 1973

Snedecor, George W. and William G. Cochran. Statistical Methods. Ames: lowa State University Press, 1972.

Stoeckel, John, Andrew A. Fisher, Mechai Viravaidya and Rachita Na Pattalung. "Maintaining Family Planning Acceptance Levels
Through Development Incentives in Northeastern Thailand." Studies in Family Planning 17, 1, January/ February 1986

Studies in Family Planning. Special Issue: Focus Group Research. Vol. 12, no. 12, Part 1, 1981

Suchman, Edward A. Evaluative Research: Principles and Practices in Public Services and Social Action Programs. New York: Russell Sage Foundation, 1967.

Sudman, Seymour. Applied Sampling. New York: Academic Press 1976.

Summers, Gene F. (ed.). Attitude Measurement. Chicago: Rand McNally \& Co., 1970.

Tanur, Judith M. (ed.). Statistics: A Guide to the Unknown. San Francisco: Holden-Day, 1972

Taylor, Howard C., Jr. and Robert J. Lapham. General Guidelines for an MCH-Based Family Planning Project. New York: The Population Council, 1974

Torgerson, Warren S. Theory and Methods of Scaling. New York Wiley, 1958

United Nations. The Methodology of Measuring the Impact of Family Planning Programmes on Fertility. Manual IX. New York United Nations, 1979

United Nations. Statistical Office. A Short Manual on Sampling. Studies in Methods, Series F. No. 9. New York: United Na tions, 1960.

Vernon, Ricardo, Gabriel Ojeda, and Marcia Townsend. "Contraceptive Social Marketing and Community Based Distribution Systems in Colombia." Studies in Family Planning 19, 6/Part 1, November/December 1988

Warwick, Donald P. and Charles A. Lininger. The Sample Survey, Theory and Practice. New York: McGraw-Hill, 1975.

Webb, Eugene J., Donald T. Campbell, Richard D. Schwartz, and Lee Sechrest. Unobtrusive Measures: Non-Respective Research in the Social Sciences. Chicago: Rand McNally, 1966.

Weiss, Carol H. (ed.). Evaluating Action Programs: Readings in Social Action and Education. Boston: Allyn and Bacon, 1972

Weiss, Carol H. Evaluation Research: Methods for Assessing Program Effectiveness. Englewood Cliffs, New Jersey: PrenticeHall, 1972.

Wishik, Samuel M. and Kwan-Hwa Chen. Couple Years of Protection. Manual 7. New York: International Institute for the Study of Human Reproduction, 1973.

World Fertility Survey. Basic Documentation Series. Nos. 1-10, Core Questionnaire and related manuals and documentation. The Hague-Voorburg: International Statistical Institute, 1975-1977

World Health Organization. Statistical Indices of Family Health. Geneva: World Health Organization, Technical Repor Series, No. 587, 1976.

Health Programme Evaluation. Geneva: World Health Organization, 1981. 



ISBN 0-87834-060-2

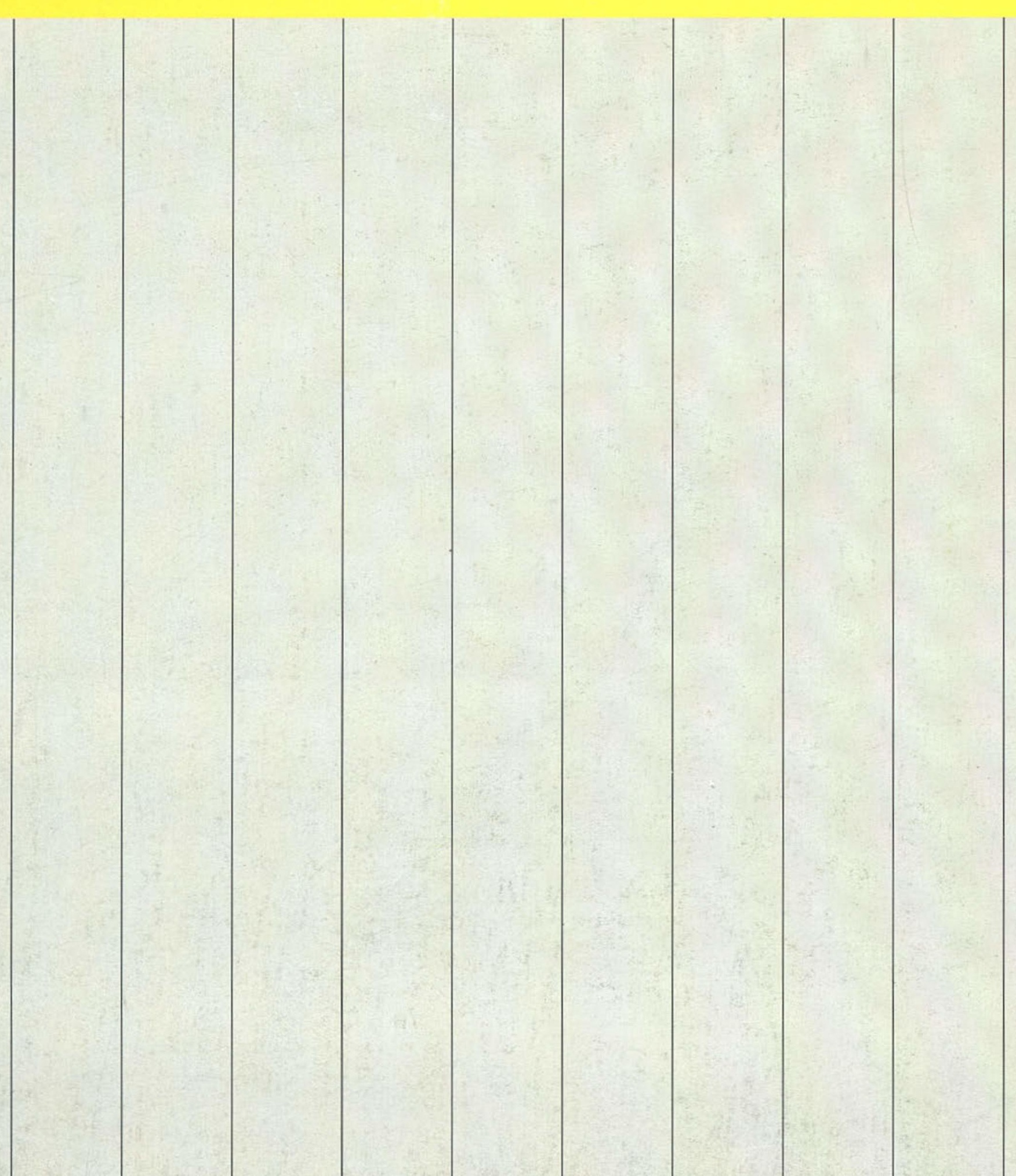

